\title{
Estimated Global, Regional, and National Disease Burdens Related to Sugar-Sweetened Beverage Consumption in 2010
}

\author{
Gitanjali M. Singh, PhD; Renata Micha, PhD; Shahab Khatibzadeh, MD; Stephen Lim, PhD; \\ Majid Ezzati, PhD; Dariush Mozaffarian, MD, DrPH; \\ on behalf of the Global Burden of Diseases Nutrition and Chronic \\ Diseases Expert Group (NutriCoDE)*
}

Background-Sugar-sweetened beverages (SSBs) are consumed globally and contribute to adiposity. However, the worldwide impact of SSBs on burdens of adiposity-related cardiovascular diseases (CVDs), cancers, and diabetes mellitus has not been assessed by nation, age, and sex.

Methods and Results - We modeled global, regional, and national burdens of disease associated with SSB consumption by age/sex in 2010. Data on SSB consumption levels were pooled from national dietary surveys worldwide. The effects of SSB intake on body mass index and diabetes mellitus, and of elevated body mass index on CVD, diabetes mellitus, and cancers were derived from large prospective cohort pooling studies. Disease-specific mortality/morbidity data were obtained from Global Burden of Diseases, Injuries, and Risk Factors 2010 Study. We computed cause-specific populationattributable fractions for SSB consumption, which were multiplied by cause-specific mortality/morbidity to compute estimates of SSB-attributable death/disability. Analyses were done by country/age/sex; uncertainties of all input data were propagated into final estimates. Worldwide, the model estimated 184000 (95\% uncertainty interval, 161 000-208000) deaths/y attributable to SSB consumption: 133000 (126000-139000) from diabetes mellitus, 45000 (26000-61 000) from CVD, and 6450 (4300-8600) from cancers. Five percent of SSB-related deaths occurred in low-income, 70.9\% in middle-income, and $24.1 \%$ in high-income countries. Proportional mortality attributable to SSBs ranged from $<1 \%$ in Japanese $>65$ years if age to 30\% in Mexicans $<45$ years of age. Among the 20 most populous countries, Mexico had largest absolute (405 deaths/million adults) and proportional (12.1\%) deaths from SSBs. A total of $8.5(2.8,19.2)$ million disability-adjusted life years were related to SSB intake (4.5\% of diabetes mellitus-related disability-adjusted life years). Conclusions-SSBs are a single, modifiable component of diet that can impact preventable death/disability in adults in high-, middle-, and low-income countries, indicating an urgent need for strong global prevention programs. (Circulation. 2015;132:639-666. DOI: 10.1161/CIRCULATIONAHA.114.010636.)

Key Words: cardiovascular diseases $\boldsymbol{\square}$ diabetes mellitus $\boldsymbol{\square}$ diet $\mathbf{\square}$ obesity

\begin{abstract}
A diposity-related chronic diseases, including type 2 diabetes mellitus, cardiovascular diseases (CVDs), and cancers, cause $>17$ million global deaths each year. ${ }^{1}$ Consumption of sugar-sweetened beverages (SSBs) increases adiposity and long-term weight gain. ${ }^{2-4}$ In addition, SSB intake appears to increase the risk of diabetes mellitus independently of adiposity, ${ }^{5}$ likely related to adverse metabolic and glucose-insulin effects. Yet, despite dramatic increases in both global sales of $\mathrm{SSBs}^{6}$ and the global pandemic of obesity, ${ }^{7,8}$ comprehensive quantitative estimates of the impact of SSB intake on
\end{abstract}

obesity-related diseases in nations worldwide by age and sex have not been available. Few published reports of countrylevel SSB consumption exist, ${ }^{9-18}$ and these previous national reports have used disparate data sources and methods that are not easily compared. In addition, previous studies have not systematically assessed how SSB intake impacts major chronic diseases worldwide by region, country, age, and sex. Comprehensive, accurate estimates of the burdens of chronic

Clinical Perspective on p 666

\footnotetext{
Received April 18, 2014; accepted April 8, 2015.
}

From Friedman School of Nutrition Science \& Policy, Tufts University, Boston, MA (G.M.S., R.M., D.M.); Department of Global Health and Population, Harvard School of Public Health, Boston, MA (S.K.); Institute for Health Metrics and Evaluation, University of Washington, Seattle (S.L.); and Departments of Global Environmental Health and Biostatistics and Epidemiology, School of Public Health, Imperial College London, United Kingdom (M.E.).

*Complete group list in the Acknowledgments.

Guest Editor for this article was Lyn M. Steffen, PhD.

Presented as a scientific abstract at the American Heart Association Scientific Sessions on Epidemiology and Prevention, New Orleans, LA, March 19, 2013.

The online-only Data Supplement is available with this article at http://circ.ahajournals.org/lookup/suppl/doi:10.1161/CIRCULATIONAHA. 114.010636/-/DC1.

Correspondence to Gitanjali M. Singh, PhD, Tufts University, Friedman School of Nutrition, 150 Harrison Ave, Boston, MA 02115. E-mail Gitanjali.Singh@Tufts.edu

(c) 2015 American Heart Association, Inc. 
obesity-related diseases attributable to SSB consumption, including the uncertainty in such estimates, are essential for informed national, regional, and global policies.

As part of our work in the 2010 Global Burden of Diseases Nutrition and Chronic Diseases Expert Group (NutriCoDE), we systematically reviewed, compiled, and extracted national data on SSB consumption worldwide and addressed issues of consistency, comparability, bias, and missingness in the collated data. We also derived and incorporated into our analysis the best available estimates of the effects of SSB intake on obesity and diabetes mellitus, and of obesity on diabetes mellitus, CVD, and cancers, including heterogeneity in these effects by age and sex. Our analysis further included data on age-, sex-, and cause-specific mortality in all nations worldwide. We used a comparative risk assessment analytic framework to quantify global, regional, and national disease burdens related to SSB consumption, assessing both the direct and obesity-mediated effects of SSBs on chronic disease.

\section{Methods}

To quantify the number of adult deaths worldwide related to SSB intake, we used a comparative risk assessment framework ${ }^{19-21}$ that captures geographical, sex, and age variation in SSB consumption, in the effects of SSB consumption on diabetes mellitus and body mass index (BMI), in the effects of BMI on disease outcomes, and in causespecific mortality (Table 1, Figure 1). We estimated both the direct effects of SSB consumption on diabetes mellitus burdens and the BMI-mediated effects of SSB consumption on CVD, diabetes mellitus, and cancer burdens.

\section{Global SSB Consumption Data by Country, Age, and Sex}

As part of the Global Burden of Diseases, Risk Factors, and Injuries 2010 study, we identified national surveys or, if unavailable, subnational surveys on SSB intake in adults through systematic searches of multiple literature databases and direct contact with experts worldwide, as described elsewhere. ${ }^{26}$ SSBs were defined as any sugarsweetened sodas, fruit drinks, sports/energy drinks, sweetened iced tea, or homemade SSBs such as frescas, which contained at least 50 kcal per 8-oz serving; $100 \%$ fruit juice was excluded. In total, we compiled data on SSBs from 62 dietary surveys including 611971 individuals conducted between 1980 and 2010 across 51 countries (Table I in the online-only Data Supplement). ${ }^{26}$ Eighty-eight percent of the surveys were nationally representative, and all but one had data on both men and women. Forty-five surveys (72\%) were from low- and middle-income countries, and 18 (28\%) were from high-income countries. Together, these surveys provided data on SSB intake in countries representing $63 \%$ of the world's adult population (Table 1) ${ }^{26}$ We also identified annual country-level data relevant to SSB intake between 1980 and 2010 for 187 countries in our analysis by using food balance sheet data on per capita sugar availability from the United Nations Food and Agriculture Organization. ${ }^{27}$

From each survey, we extracted data on survey location, time period, representativeness, sampling design, dietary assessment method, and the age- and sex-specific distributions (mean, standard deviation) of SSB intake and corresponding strata-specific sample size, using a standardized electronic spreadsheet. We evaluated the quality of population sampling and diet assessment methods and checked data for plausibility. SSB consumption data were adjusted for total energy by using the residual method to reduce measurement error and account for differences in physical activity and metabolic rate. ${ }^{28}$

To combine individual-level intake data with country-level food availability data, to address issues of data incomparability, and to capture the uncertainty in estimates of beverage intake attributable to measurement error, sampling uncertainty, and modeling uncertainty, we used established age-integrating Bayesian hierarchical modeling methods. The primary model inputs were survey-level quantitative data, including country-, time-, age-, and sex-specific consumption levels (mean and standard deviation); data on the numbers of subjects in each strata; survey-level indicator covariates for sampling representativeness, dietary assessment method, and type of dietary metric; country-level year-specific data relevant to sugar availability from the United Nations Food and Agriculture Organization; and country, region (21 regions), and superregion ( 7 groupings of regions) random effects (Table 1). ${ }^{19,26}$ Model outputs were country-, age-, sex-, and year-specific estimates of mean SSB consumption and its uncertainty. In our present analysis, we used data on SSB consumption produced by this model for the year 2010 .

\section{Direct and BMI-Mediated Effects of SSB Intake on CVD, Diabetes Mellitus, and Cancers}

We incorporated into our analysis the best available estimates for etiologic effects of SSBs on BMI and type 2 diabetes mellitus and of BMI on CVD (ischemic heart disease, stroke), type 2 diabetes mellitus, and cancers (esophageal, colon, pancreatic, breast, uterine, kidney, gall bladder; Tables 1 and 2)..$^{24,29}$

\section{Effects of SSB Intake on BMI}

Long-term effects of SSB intake on BMI in adults were derived from meta-analysis of 3 large prospective US cohort studies that have evaluated the effects of changes in SSB intake on changes in weight gain, namely, the Nurses' Health Study, the Nurses' Health Study II, and the Health Professionals Follow-up Study, with a combined sample size of 120877 participants (Table 1). Each serving per day increase in SSB intake was associated with a 0.10 (95\% confidence interval [CI], 0.05-0.15) kg/m² increase in BMI in individuals with BMI $<25$ and a $0.23(95 \% \mathrm{CI}, 0.14-0.32) \mathrm{kg} / \mathrm{m}^{2}$ increase in BMI in individuals with $\mathrm{BMI} \geq 25$ (Table 2). ${ }^{29}$ These estimates are consistent with a recent meta-analysis of 7 prospective cohorts (174252 individuals) that reported an 0.12- to $0.22-\mathrm{kg}$ increase in weight per serving per day of SSBs over a 1-year period, and a meta-analysis of 5 trials (292 participants) that found a $0.85-\mathrm{kg}(95 \% \mathrm{CI}, 0.50-1.2 \mathrm{~kg})$ increase in body weight when SSBs were added to the diet. ${ }^{30}$ These estimates are also broadly supported by results from 2 recent large randomized trials of the effects of SSB intake on weight gain in children, one of which reported BMI reductions of $\sim 0.57 \mathrm{~kg} / \mathrm{m}^{2}$ for a 1.7 servings $/ \mathrm{d}$ reduction in SSB intake over a 1-year period, and the other of which reported significant reductions in weight gain, body fat change, and BMI $z$ score for each 104-kcal reduction in sugary beverage intake per day over an 18-month period. ${ }^{2,3}$

\section{Effects of SSB Intake on Diabetes Mellitus}

Effects of SSB consumption on diabetes mellitus were based on a meta-analysis of 8 prospective cohorts with a total of 310819 participants and 15043 cases of type 2 diabetes mellitus (Tables 1 and 2). ${ }^{31}$ In this meta-analysis, individuals in the highest category of SSB intake (1-2 servings/d) had a $26 \%$ greater risk of developing type 2 diabetes mellitus in comparison with those in the lowest category of SSB intake (none or $<1$ serving per month; risk ratio, 1.26; $95 \% \mathrm{CI}$, 1.12-1.41). The association between SSB intake and risk of type 2 diabetes mellitus in this meta-analysis was consistent across sex and ethnic groups, which included blacks, whites, and Asians. Although there was heterogeneity among studies $\left(I^{2}=66 \%\right)$, all but one showed positive associations between SSB intake and risk of type 2 diabetes mellitus, with the strength of the association increasing with study size and duration. Three cohorts included adjustment for BMI, most appropriate for our modeling of direct (nonobesity-mediated) effects, but these also adjusted for total energy intake, which could result in underestimation of true effects. Results from this meta-analysis are supported by other large studies, including a subcohort of the European Prospective Investigation into Cancer and Nutrition, which included 15374 participants and 11684 cases of type 2 diabetes mellitus, and reported a hazard ratio of 1.18 (95\% CI, 1.06-1.32) for the association of SSBs with type 2 diabetes mellitus. ${ }^{22,32}$ 


\section{Sensitivity Analyses}

To determine whether relative risks for individual dietary components derived from prospective cohorts might overestimate the impact on chronic disease attributable to residual confounding by other dietary factors, we performed 3 sensitivity analyses based on studies of overall dietary patterns. ${ }^{29}$ In brief, we compared the predicted risk of coronary heart disease (CHD) calculated from the effects of individual dietary components on CHD from cohort data with the observed risk of CHD in dietary pattern studies, including (1) prospective cohort studies on the association of overall dietary patterns with incident CHD, (2) randomized controlled feeding trials quantifying the effects of overall dietary patterns on SBP and low-density lipoprotein cholesterol, and (3) a large randomized clinical trial evaluating the impact of an overall dietary pattern on incident CVD events.

\section{Effects of BMI on CVD, Diabetes Mellitus, and Cancers}

Evidence for effects of BMI on CVD, diabetes mellitus, and sitespecific cancers was obtained from published analyses of large international pooling studies ${ }^{24,25}$ (Tables 1 and 2). The effects of BMI on CVD and diabetes mellitus were obtained from pooled analysis of 163 international cohorts with 2.43 million participants and 70000 cases. At the reference median age of 60 , the relative risk of BMI on CVDs, such as ischemic heart disease, stroke, and hypertensive heart disease ranged from 1.44 (95\% CI, 1.40-1.48) to 1.90 (95\% CI, 1.17-3.07) per $5 \mathrm{~kg} / \mathrm{m}^{2}$ increase in BMI, and the corresponding pooled effect for diabetes mellitus was 2.32 (95\% CI, 2.04-2.63). The effects of BMI on 7 site-specific cancers (breast, uterine, esophageal, pancreatic, colon, kidney, and gall bladder) were based on pooled analysis of 221 international cohorts with 282137 cases..$^{25}$ The effects of SSB on CVD and cancers were assumed to be mediated only through changes in BMI, whereas the effects of SSB on diabetes mellitus were assumed to be mediated through both BMI- and non-BMIrelated pathways. ${ }^{31,33-35}$

A J-shaped relationship between BMI and all-cause mortality has been observed in some population-based studies, and such nonlinearities are primarily attributable to effect modification by smoking or reverse causation attributable to underlying chronic disease (such as chronic respiratory disease or cancer) in which weight loss may precede death by a decade or more. Among the international pooling projects used in estimating the etiologic effects of BMI on CVD and diabetes mellitus for the present analysis, $21 \mathrm{~kg} / \mathrm{m}^{2}$ was the lowest empirically observed minimum beyond which no additional benefits of lowering BMI were found. ${ }^{24}$ In our analyses, we therefore restricted benefits of SSB reduction up to a BMI of $21 \mathrm{~kg} / \mathrm{m}^{2}$, beyond which we did not include additional benefits of further weight reduction.

We used the same risk estimates in men and women and across different races based on evidence that proportional effects of BMI are generally similar by sex and race, ${ }^{24,36}$ with the exception of specific cancers for which we used separate risk estimates in men versus women based on evidence for differing effects of BMI by $\operatorname{sex}^{25}$ (Table 2). In our analysis, we included age-specific relative risks for the effects of BMI on CVD and diabetes mellitus, based on previous work demonstrating decreasing proportional effects at older ages. ${ }^{24}$

\section{Cause-Specific Mortality by Country, Age, and Sex}

Data on mortality from 235 causes were compiled as part of the 2010 GBD study (Table 1). ${ }^{1}$ In brief, data were obtained on causes of death for 187 countries from 1980 to 2010 based on vital registration, verbal autopsy, mortality surveillance, censuses, surveys, hospitals, police records, and mortuaries; and assessed for completeness, diagnostic accuracy, missing data, stochastic variations, and probable causes of death. As described elsewhere, statistical modeling strategies estimated cause-specific mortality, including different permutations of covariates and assessment of model performance with specific models selected based on data quality and out-of-sample testing of prediction error and the validity of $95 \%$ uncertainty intervals (UIs). ${ }^{1}$ Cause-specific mortality fractions within each age-sex group were constrained to sum to total mortality based on draws from the uncertainty distributions. The final mortality data set included cause-, age-, and sex-specific mortality for 187 countries between 1990 and 2010. In this analysis we used age-, sex-, and country-specific data for 2010 on deaths attributable to diabetes mellitus (E10-E14), ischemic heart disease (International Classification of Diseases, Tenth Revision codes I20-I25), ischemic stroke (I63, I65-I67, I69.3), hypertensive heart disease (I11), and breast (C50), uterine (C54-C55), esophageal (C15), pancreatic (C25), colon (C18-C21), kidney (C64), and gall bladder (C23) cancer.

\section{Statistical Analysis}

\section{Estimation of Deaths Attributable to SSB Consumption}

We quantified disease burdens attributable to SSB consumption in 2010 in 187 countries by age and sex by incorporating the data described above into a comparative risk assessment analytic framework (Figure 1). We included in our analysis the direct effects of SSB on diabetes mellitus and BMI, and the BMI-mediated effects of SSBs on diabetes mellitus, CVD (CHD, stroke), and cancers (breast, uterine, esophageal, colon, pancreatic, kidney, and gall bladder), as well. All analyses evaluated SSB consumption, effects of SSBs on diabetes mellitus and BMI, effects of BMI on diseases, and causespecific mortality across 16 age- and sex-specific strata within each country (men and women ages 20-24, 25-34, 35-44, 45-54, 55-64, $65-74,75-84$, and $85+$ years). We also accounted for stronger effects of SSB intake on BMI in overweight versus normal adults ${ }^{29}$ by partitioning effects in each age-, sex-, and country-specific strata according to the proportion of the population who were normal weight or overweight. ${ }^{7,8}$ Our analyses did not involve ecological correlations (ie, simple comparisons of SSB intakes and disease rates) because these would be limited by substantial confounding. We have instead quantitatively assessed and incorporated into our analysis age- and sex-specific data on SSB consumption, external evidence on causal effects of SSBs on disease risk, and validated data on country-, age-, sex-, and cause-specific mortality.

For diabetes mellitus and each BMI-related disease end point, we calculated the disease-specific population-attributable fraction attributable to SSB consumption:

$$
\frac{\int_{x=0}^{m} R R(x) P(x) d x-\int_{x=0}^{m} R R(x) P^{\prime}(x) d x}{\int_{x=0}^{m} R R(x) P(x) d x}
$$

where $x=\mathrm{SSB}$ consumption level; $P(x)=$ current distribution in the age and sex stratum; $P^{\prime}(x)=$ alternative distribution (zero consumption); $R R(x)=$ relative risk of mortality at SSB consumption level $x$; and $m=$ maximum exposure level. Mortality attributable to SSB consumption was calculated by multiplying the calculated population-attributable fraction by the observed number of causespecific deaths (Figure 1). Analyses were done separately for each country, age, and sex group, and assessed as both absolute and proportional mortality.

\section{Estimation of DALYs Attributable to SSB Consumption}

Disability-adjusted life-years (DALYs) are summary metrics of population health that measure how many years of healthy life are lost because of death and disability. ${ }^{37}$ DALYs are the sum of 2 components: the years of life lost attributable to premature mortality and the years lived with disability. Years of life lost are computed by multiplying the number of deaths at each age by a standard life expectancy at that age in the given population. Years lived with disability are estimated as the prevalence of different disease sequelae multiplied by the disability weight for each sequela. ${ }^{38}$ Disability weights are selected on the basis of surveys of the general population about the loss of health associated with the health state related to the disease sequelae..$^{39}$ The estimation of cause-specific DALYs by country, age, and sex has been described elsewhere. ${ }^{37-39}$ DALYs attributable to SSB consumption were calculated by multiplying the total DALYs attributable to CVD, diabetes mellitus, and cancers by the country-, 
Table 1. Description of Data Sources and Modeling Methods Used to Estimate Adult SSB Consumption Levels, the Effects of SSB Intakes on BMI and Diabetes Mellitus, and Total Cause-Specific Mortality by Country, Age, and Sex

Data Source and Description

SSB consumption by country, age, and sex

Total consumption of sugar-sweetened beverages containing $>50 \mathrm{kcal} / 8-0 z$ serving, including sodas, fruit drinks, sports/energy drinks, and sweetened iced tea

Relative risks by age and sex

Effects of SSB on diabetes mellitus

Published meta-analyses of 8 prospective cohort studies
Data Coverage

\section{Individual-level survey data}

A total of 780 age- and sex-specific data points, $88 \%$ nationally representative, were collected from 51 countries and represented $63 \%$ of the world's adult population. $16 \%$ of all data were from multiple dietary recall surveys, $20 \%$ of all data were from food-frequency questionnaires, $17 \%$ of data were from single dietary recall surveys, and $47 \%$ of data were from household availability surveys.
National food availability data

Total annual per capita sugar availability in each of 187 countries worldwide based on availability of sugar, sugar beet, sugar cane, noncentrifugal sugar, and sugar crops. These data are based on U.N. Food and Agriculture Organization (FA0) food balance sheets which capture a country's net annual food availability, accounting for imports and exports.
Data were from US, European, and Asian cohorts including 310819 participants and 15043 cases of type 2 diabetes mellitus.*

\section{Linear effects of SSB on BMI}

Original meta-analysis of 3 prospective cohort studies (NHS I, NHS II, HPFS)

Effects of BMI on CVD and diabetes mellitus Published pooled analysis of 3 international pooling projects (APCSC, PSC, ERFC)

Effects of BMl on site-specific cancers

Published meta-analysis of 221 cohort studies.

Data were from North American, East Asian, European, and Australian cohorts with a total of 282137 incident cases of cancer $>133000000$ person-years of follow-up. ${ }^{25}$

Cause-specific total mortality by country, age, and sex Vital registration with medical certification of cause of death

Verbal autopsy (sample registration, demographic surveillance systems)

Cancer registries

Survey/census data

Sibling history

Burial/mortuary data

Hospital records

Data were from East Asian, North American, and European pooling projects comprising a total of 163 cohorts, 2.43 million participants, and 70000 CVD events. ${ }^{24}$

\section{Data represented 2798 site-years from 130 countries}

Data represented 486 site-years from 66 countries, 10\% nationally representative

Data represented 2715 site-years from 93 countries

Data were from 56 national surveys

Data represented 1557 survey-years from 61 countries

Data represented 32 site-years from 11 countries

Data represented 21 site-years

Data represented 1129 site-years from 122 countries

Police records

These represent the primary data used in analyses to compute mortality attributable to SSB consumption by age, sex, and country in 2010. BMI indicates body mass index; CVD, cardiovascular disease; MCMC, Markov chain Monte Carlo; and SSB, sugar-sweetened beverages.

${ }^{*}$ Malik et al (2010). ${ }^{5} \mathrm{~A}$ meta-analysis of 8 prospective cohorts (310819 participants, 15043 incident cases of diabetes mellitus), comparing the highest (1-2 servings/d) vs lowest $(<1$ serving/mo) category of intake. Three cohorts included adjustment for BMI, most appropriate for our modeling of direct (non-obesitymediated) effects; but these also adjusted for total energy intake, which could result in underestimation of true effects. A more recent meta-analysis of 6 cohorts, published after finalization of our dietary RRs, reported a similar pooled dose-response association per daily SSB serving of $1.20(95 \% \mathrm{Cl}, 1.12-1.29){ }^{22}$ 
DisMod3, ${ }^{19}$ a Bayesian hierarchical method was used to pool data from multiple sources and model missing data using informative time-varying covariates, borrowing information across geographical regions and time periods while also incorporating uncertainty attributable to measurement error and model specification. Models were fit by using a randomized MCMC algorithm based on the Adaptive Metropolis step function.

Systematic review and meta-analysis were used to identify and pool relevant data from cohort studies. Given that the effects of SSB on incidence of diabetes mellitus are attenuated with age, we extrapolated the effect estimate into 10 -y age groups from age 20 to 100 using an age pattern derived from the average percent change in relative risk for CVD and diabetes mellitus across 4 metabolic risk factors.

The association of change in BMI with change in SSB consumption was assessed by using multivariate linear regression accounting for within-person repeated measures. Results across the 3 cohorts were pooled by an inverse-variance weighted meta-regression as described in earlier work. ${ }^{23}$ Separate linear effects were estimated for $\mathrm{BMl}<25$ and $\mathrm{BMI} \geq 25$ because the rate of increase in $\mathrm{BMl}$ attributable to SSB intake varies based on an individual's baseline BMI.

Relative risks from the pooling projects were interpolated and extrapolated into standard age groups by using log-linear models. Age-specific relative risks were pooled using randomeffects models. Trends in age-specific relative risks from pooled analyses were compared with trends in original cohort data to ensure validity of pooled results.

Systematic review and random-effects meta-analysis

Cause of Death Ensemble Modeling (CODEm), a modeling strategy encompassing 4 families of statistical models, was used to pool mortality data from diverse sources, aggregate deaths hierarchically, and capture uncertainty attributable to model parameter estimation, model specification, and fundamental sources of error.
Both study-specific and national-level covariates were incorporated in the model. Study-level covariates included information on national representativeness of data points, method/metric of data collection, and sex. Country-level information included country, region, and superregion random effects. Other country-level covariates such as gross domestic product were tested but did not improve prediction.

Included studies considered adjustment for potential confounding by age, sex, and various lifestyle factors, such as smoking, alcohol use, and physical activity, and various dietary habits. For most, a positive association persisted, suggesting an independent effect of SSBs on incident diabetes mellitus.

Analyses were adjusted for age, baseline BMI, and changes in other lifestyle behaviors such as diet, smoking, physical activity, alcohol consumption, sleep duration, and TV watching.

Effect modification by race/ethnicity and sex were assessed but were not found to be statistically significant.

Effect modification by race/ethnicity, sex, and age were assessed for cancers other than breast and ovarian, but were not found to be statistically significant.

Covariates were selected from a database of mortality predictors based on the cause of death being modeled. Covariates were tested for predictive ability before inclusion in a given model.
Models were assessed for convergence of MCMC iterations and were validated by using goodness-

of-fit tests. Final model results were assessed for plausibility by subject-matter experts.

Standard methods of assessing publication bias, such as Begg and Eggers tests and visual inspection of funnel plots indicated no evidence of such bias in this analysis. Cochrane $Q$ test and the $\mathcal{P}$ statistic revealed statistically significant heterogeneity, which was reduced in sensitivity analyses excluding smaller studies of shorter duration.

Change in SSB consumption resulted in a statistically significant change in BMl across all 3 cohorts, and the magnitude of change in $\mathrm{BMI}$ was consistent across all cohorts. This is the only analysis thus far to examine effects of change in SSB consumption on change in adiposity.

Trends in age-specific relative risks from pooled analyses were compared with trends in original cohort data to ensure validity of pooled results. The $R$ test did not reveal significant heterogeneity between studies for any age group.

Between-study heterogeneity varied by cancer, from low to moderate $(0 \%-55 \%)$, and there was no evidence of publication bias in inspection of funnel plots.

Models were validated by using out-ofsample predictive validity tests in which $30 \%$ of data were withheld from initial model fits. Predicted trends were then compared with trends in the existing held-out data. 


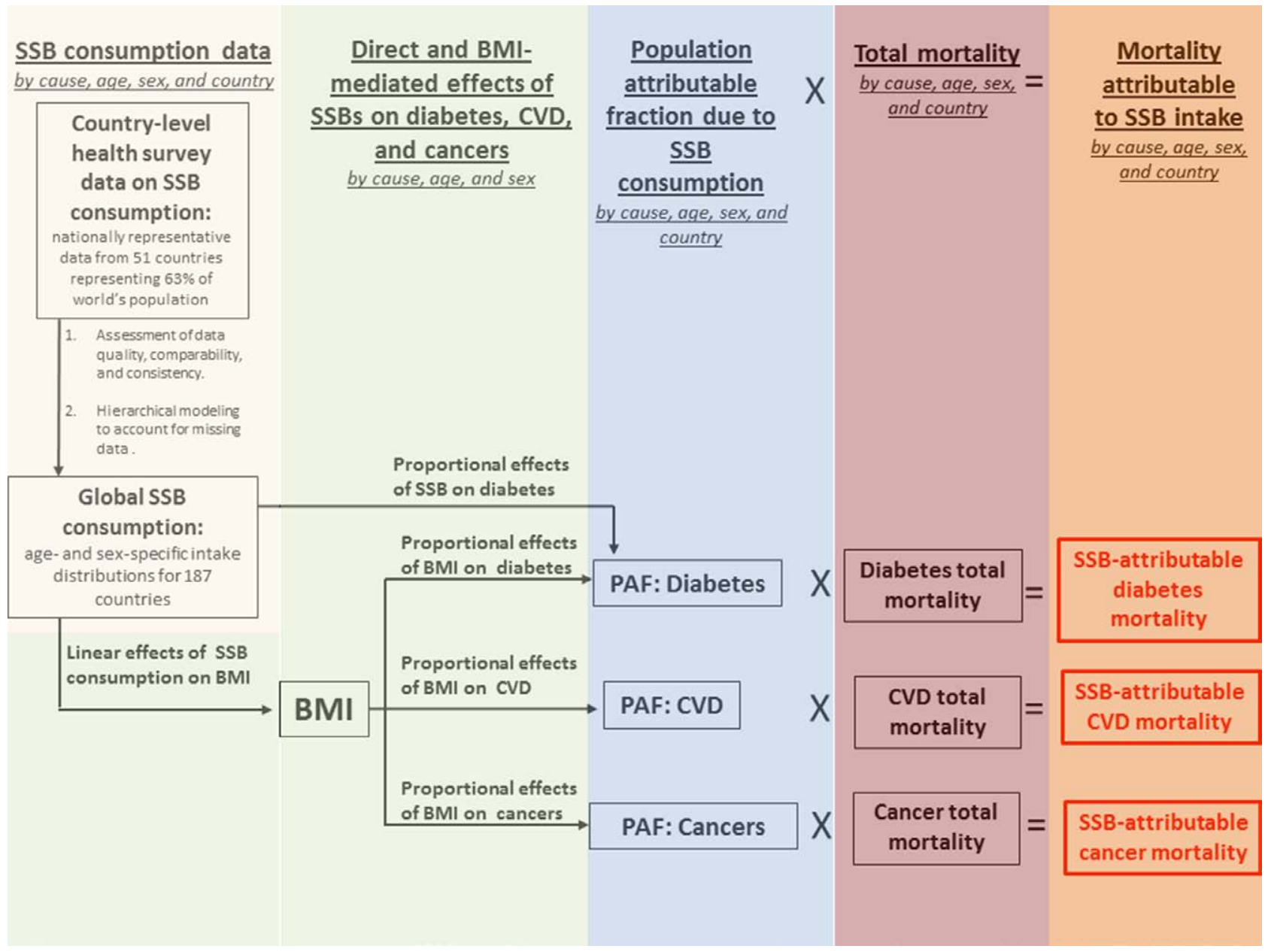

Figure 1. Schematic diagram of the relationships between the data sources used in the comparative risk assessment modeling framework on which this analysis is based. BMI indicates body mass index; CVD, cardiovascular disease; PAF, population-attributable fraction; and SSB, sugar-sweetened beverages.

age-, and sex-specific population-attributable fractions, which were calculated as described above. ${ }^{37,38}$

\section{Estimation of Uncertainty}

We used Monte Carlo simulation to quantify the uncertainty in the attributable deaths and DALYs, propagating the uncertainty from SSB intake data (which includes both measurement and sampling error and modeling uncertainty), and uncertainty from the relative risks into our final estimates. We drew 1000 times from the distribution of SSB consumption for each country-age-sex group as characterized by its mean and standard error. For each mean exposure, population-representative standard deviations were predicted by using coefficients from regressions performed on all available dietary survey data in our collection, in which standard deviation was the dependent variable and mean was the independent variable. ${ }^{40,41}$ Independent of exposure, we generated 1000 draws of the etiologic relative risks of SSB intake on diabetes mellitus and BMI, and of BMI on adiposity-related diseases. These simultaneous draws were entered into the analysis to generate 1000 mortality estimates for each country-age-sex group, of which we report the mean and 95\% UIs. All analyses were performed by using R software, version 2.15.0.

\section{Results}

In 2010, the mean global consumption of SSBs among adults was 0.58 servings/d (95\% UI, 0.37-0.89). The mean intake varied substantially in men and women across different ages and world regions, highest in countries in Latin America and the Caribbean and lowest in parts of East Asia. Detailed findings on SSB consumption patterns across the world have been reported. ${ }^{26}$

\section{Mortality Related to SSB Consumption}

\section{Global Mortality Related to SSB Consumption}

In 2010, the model attributed a total of 184000 (95\% UI, 161000208000) deaths globally to SSB intake (Table 3), representing $5.3 \%$ (95\% UI, 5.0\%-5.5\%) of all diabetes mellitus deaths, $0.4 \%$ (95\%UI, $0.3 \%-0.6 \%$ ) of BMI-related CVD deaths, and $0.3 \%$ (95\% UI, $0.2 \%-0.3 \%)$ of all BMI-related cancer deaths. Most of these deaths $(72.3 \%$ or $133000 ; 95 \%$ UI, $126000-139000)$ were attributable to diabetes mellitus, $24.2 \%$ (45000; 95\% UI, 29000-61 000) were attributable to CVD, and 3.5\% (6450; $95 \%$ UI, 4300-8600) were attributable to BMI-related cancers. Half of all SSB-attributable deaths were in women. Three in $4(75.9 \%)$ of all deaths attributable to SSB consumption occurred in lowand middle-income countries. Globally, absolute mortality from SSBs was greatest in adults $>65$ years of age, at 167 (95\% UI, 141-195) deaths per million adults. Conversely, proportional mortality was highest among adults aged 20 to 44 years, in whom $14.0 \%$ (95\% UI, $12.9 \%-15.0 \%)$ of all diabetes mellitus- and adiposity-related deaths were attributable to SSB consumption. 


\section{Regional Mortality Related to SSB Consumption}

Across 9 major world regions, Latin America and the Caribbean had the highest absolute mortality related to SSB consumption (48000 per million adults; 95\% UI, 4100054000), whereas Australia and New Zealand had the lowest (560; 95\% UI, 440-700; Table 3). Among age and sex groups in these regions, men $>65$ years in Latin America and the Caribbean had the highest SSB-related absolute mortality (582 deaths per million men; 95\% UI, 485-681); women $>65$ years of age in this region were close behind ( 552 deaths per million women; 95\% UI, 460-644). Adults 65+ years in the United States and Canada had the next highest SSB-attributable mortality: 379 (95\% UI, 285-478) per million men and 300 (95\% UI, 227-386) per million women. Women aged 20 to 44 years in Western Europe and Australia/New Zealand had the lowest mortality: 2 (95\% UI, 1-3) deaths per million.

Although older adults had the highest absolute mortality attributable to SSBs, younger adults had the highest proportional mortality (Table 3). Among adults aged 20 to 44 years in Latin America/Caribbean, 1 in 7 (13.9\%; 95\% UI, 11.7\%$16.1 \%$ ) of all diabetes mellitus- and adiposity-related deaths in men and 1 in 9 (10.9\%; 95\% UI, 9.3\%-12.4\%) of all such deaths in women were attributable to SSBs. Considering only deaths attributable to diabetes mellitus, 1 in 3 such deaths in men aged 20 to 44 years in both Latin America/Caribbean and United States/Canada were attributable to SSBs. Among all diabetes mellitus deaths, the percentage related to SSB consumption exceeded $10 \%$ among adults aged 20 to 44 years in every world region except South Asia.

\section{National Mortality Related to SSB Consumption}

Across all countries worldwide, proportional mortality related to SSB intake varied substantially (Figure 2, Table II in the online-only Data Supplement). Proportional deaths were highest in Mexican men aged 20 to 44 years, in whom 1 in 3 (33.6; 95\% UI, 26.4-39.5\%) diabetes mellitus- and BMIrelated deaths were linked to SSB consumption. Proportional mortality also exceeded $20 \%$ in adults aged 20 to 44 years in Kiribati, Gabon, Marshall Islands, Belize, Barbados, and Tonga. Proportional mortality attributable to SSBs was lowest in adults aged $>65$ years in several East Asian countries, approaching zero in some cases.

Among larger nations (population $>1$ million), the SSBrelated mortality rate was highest in Mexico (405 deaths per million adults; 95\% UI, 345-462) and lowest in Bangladesh (1; 95\% UI, 0-2 deaths per million adults; Table II in the online-only Data Supplement). In total, Mexico had an estimated 24000 (95\% UI, 21000-28000) SSB-related deaths in 2010, whereas Bangladesh had 72 (95\% UI, 40-104) such deaths. Among the 20 countries with highest SSB-related deaths, at least 8 of these countries were in Latin America and the Caribbean across all age and sex subgroups (Figure 3).

Of the 20 most populous countries in the world, the death rate related to SSB intake was highest in Mexico in all age-sex groups, followed by the United States, Indonesia, and Brazil (Figure I in the online-only Data Supplement). In these 20 countries, most SSB-related deaths were attributable to diabetes mellitus, except in Russia and Egypt in which most SSBrelated deaths were attributable to CVD. Overall, the United
States ranked second in SSB-related deaths among the 20 most populous countries, with an absolute death rate of 125 (95\% UI, 101-149) per million adults, or 25000 total deaths (95\% UI, 20000-30000) related to SSB consumption in 2010 (Table II in the online-only Data Supplement).

\section{Morbidity Related to SSB Consumption}

\section{Global DALYS Related to SSB Consumption}

In 2010, a total of 8526456 (95\% UI, 2769953-19244657) DALYs were attributable to SSBs, of which $49.5 \%$ were attributable to CVD, $41.4 \%$ were attributable to diabetes mellitus, $4.5 \%$ were attributable to BMI-related cancers, and $4.9 \%$ were attributable to musculoskeletal disorders (Table 4 and Table III in the online-only Data Supplement.). Globally, 0.7\% (95\% UI, $0.3 \%-1.5 \%$ ) of total DALYs were related to SSB consumption; $1.6 \%$ (95\% UI, $0.5 \%-4.5 \%$ ) of CVD DALYs, $4.5 \%(95 \%$ UI, $1.7 \%-8.9 \%$ ) of diabetes mellitus DALYs, $0.2 \%$ (95\% UI, $0.1 \%-0.7 \%)$ of obesity-related cancer DALYs, and $0.4 \%(95 \%$ UI, $0.1 \%-1.3 \%$ ) of musculoskeletal DALYs were attributable to intake of SSBs. Lower- and middle-income countries had the largest absolute number of SSB-related DALYs, a total of 4243602 (95\% UI, 998925-9058923) DALYs, or 2931 (95\% UI, 690-6258) per million adults. Upper- and middle-income countries had the highest percentage of diabetes mellitusrelated DALYS that were related to SSB consumption $(6.1 \%$; 95\% UI, 2.5-11.7; Table 4).

As with SSB-related mortality, the absolute number of DALYs attributable to SSBs was highest in men $>65$ years of age, at 6230 (95\% UI, 2300-16095) DALYs per million. Men $<45$ years of age had the highest percentage of CVD-related DALYs caused by SSBs $(2.9 \%$; 95\% UI, 1.0\%-6.9\%), and the highest percentage of diabetes mellitus-related DALYS caused by SSBs $(6.1 \%$; 95\% UI, 2.3\%-12.1\%).

\section{Regional DALYs Related to SSB Consumption}

Among 9 world regions, the greatest absolute SSB-related burdens were in South Asia, with 3528 (95\% UI, 686-7070) DALYs per million adults and United States/Canada (3265; $95 \%$ UI, 1934-4982), whereas Australia/New Zealand had the least (609; 95\% UI, 364-1028). In Latin America/ Caribbean, almost 1 in 10 diabetes mellitus-related DALYs were related to SSB consumption $(9.7 \%$; $95 \%$ UI, 4\%$18.3 \%$ ), which also accounted for $\sim 1$ in 12 diabetes mellitus-related DALYs in United States/Canada (8.5\%; 95\% UI, 3.9\%-14.3\%). The percentage of all diabetes mellitusrelated DALYs caused by SSB consumption was highest in men $<45$ years of age in United States/Canada $(12.9 \%$; $95 \%$ UI, 5.8\%-21.8\%) and Latin America/Caribbean (12.4\%; $95 \%$ UI, $5.0 \%-23.2 \%$ ).

\section{National DALYs Related to SSB Consumption}

Of the world's 20 most populous countries, Mexico had the greatest number of SSB-attributable DALYs per million adults (3960; 95\% UI, 1516-13990), whereas China had the lowest (584; 95\% UI, 42-2462). In Mexico, almost 1 of every 6 diabetes mellitus-related DALYs was attributable to SSB intake (15.8\%; 95\% UI, 7.0\%-27.5\%). In the United States, a total of 32997 (95\% UI, 2279-73697) DALYs were related to SSB consumption in 2010, or 2087 (95\% UI, 2050-5180) per 
Table 2. Sources and Magnitudes of the Effects of SSBs on Diabetes Mellitus, SSB on BMI, and BMI on Chronic Disease Outcomes

\begin{tabular}{|c|c|c|c|c|}
\hline Risk Factor & $\begin{array}{l}\text { Type of Effect } \\
\text { Estimate }\end{array}$ & Source of Effect Estimate & Unit of Effect Estimate & Sex \\
\hline SSB-diabetes mellitus & Relative risk & $\begin{array}{c}\text { Published meta-analysis of } 8 \text { prospective } \\
\text { cohort studies }{ }^{\star}\end{array}$ & Per SSB serving/d & Both \\
\hline SSB-BMI (for baseline BMI < 25) & Linear effect & $\begin{array}{c}\text { Original meta-analysis of } 3 \text { prospective cohort } \\
\text { studies }\end{array}$ & $\begin{array}{l}\mathrm{kg} / \mathrm{m}^{2} \text { increase in BMl per } \\
\text { SSB serving } / \mathrm{d}\end{array}$ & Both \\
\hline SSB-BMI (for baseline BMI $\geq 25$ ) & Linear effect & $\begin{array}{c}\text { Original meta-analysis of } 3 \text { prospective cohort } \\
\text { studies }\end{array}$ & $\begin{array}{l}\mathrm{kg} / \mathrm{m}^{2} \text { increase in BMl per } \\
\text { 8-0z SSB serving } / \mathrm{d}\end{array}$ & Both \\
\hline BMI-ischemic stroke & Relative risk & $\begin{array}{l}\text { Pooled analysis of APCSC, PSC, and ERFC } \\
\text { international pooling projects }{ }^{24}\end{array}$ & Per $\mathrm{kg} / \mathrm{m}^{2}$ increase in BMl & Both \\
\hline BMI-ischemic heart disease & Relative risk & $\begin{array}{l}\text { Pooled analysis of APCSC, PSC, and ERFC } \\
\text { international pooling projects }{ }^{24}\end{array}$ & Per $5 \mathrm{~kg} / \mathrm{m}^{2}$ increase in BMl & Both \\
\hline BMI-hypertensive heart disease & Relative risk & $\begin{array}{l}\text { Pooled analysis of APCSC, PSC, and ERFC } \\
\text { international pooling projects }{ }^{24}\end{array}$ & Per $5 \mathrm{~kg} / \mathrm{m}^{2}$ increase in BMl & Both \\
\hline BMI-diabetes mellitus & Relative risk & $\begin{array}{l}\text { Pooled analysis of APCSC, PSC, and ERFC } \\
\text { international pooling projects }{ }^{24}\end{array}$ & Per $5 \mathrm{~kg} / \mathrm{m}^{2}$ increase in BMl & Both \\
\hline BMI-breast cancer & Relative risk & Meta-analysis of 221 cohort studies ${ }^{25}$ & Per $5 \mathrm{~kg} / \mathrm{m}^{2}$ increase in BMl & Female \\
\hline BMI-colon cancer & Relative risk & Meta-analysis of 221 cohort studies ${ }^{25}$ & Per $5 \mathrm{~kg} / \mathrm{m}^{2}$ increase in BMl & $\begin{array}{c}\text { Female } \\
\text { Male }\end{array}$ \\
\hline BMI-pancreatic cancer & Relative risk & Meta-analysis of 221 cohort studies ${ }^{25}$ & Per $5 \mathrm{~kg} / \mathrm{m}^{2}$ increase in BMl & $\begin{array}{c}\text { Female } \\
\text { Male }\end{array}$ \\
\hline BMI-esophageal cancer & Relative risk & Meta-analysis of 221 cohort studies ${ }^{25}$ & Per $5 \mathrm{~kg} / \mathrm{m}^{2}$ increase in BMl & $\begin{array}{c}\text { Female } \\
\text { Male }\end{array}$ \\
\hline BMI-uterine cancer & Relative risk & Meta-analysis of 221 cohort studies ${ }^{25}$ & Per $5 \mathrm{~kg} / \mathrm{m}^{2}$ increase in BMI & Female \\
\hline BMI-kidney cancer & Relative risk & Meta-analysis of 221 cohort studies ${ }^{25}$ & Per $5 \mathrm{~kg} / \mathrm{m}^{2}$ increase in BMl & $\begin{array}{c}\text { Female } \\
\text { Male }\end{array}$ \\
\hline BMI-gall bladder cancer & Relative risk & Meta-analysis of 221 cohort studies ${ }^{25}$ & Per $5 \mathrm{~kg} / \mathrm{m}^{2}$ increase in BMl & $\begin{array}{c}\text { Female } \\
\text { Male }\end{array}$ \\
\hline
\end{tabular}

BMI indicates body mass index; $\mathrm{Cl}$, confidence interval; CVD, cardiovascular disease; and SSB, sugar-sweetened beverages.

${ }^{*}$ Malik et al (2010). ${ }^{5}$ A meta-analysis of 8 prospective cohorts (310 819 participants, 15043 incident cases of diabetes mellitus), comparing the highest (1-2 servings/d) vs lowest $(<1$ serving/mo) category of intake. Three cohorts included adjustment for BMl, most appropriate for our modeling of direct (nonobesitymediated) effects; but these also adjusted for total energy intake, which could result in underestimation of true effects. A more recent meta-analysis of 6 cohorts, published after finalization of our dietary RRs, reported a similar pooled dose-response association per daily SSB serving of $1.20(95 \% \mathrm{Cl}, 1.121 .29) .{ }^{22}$

million adults, and 1.1\% (95\% UI, 0.6\%-1.6\%) of all DALYs in the United States were attributable to SSBs.

\section{Discussion}

These findings, based on a model that incorporated individuallevel national surveys of SSB consumption, country-level food availability data, the adiposity-mediated and direct effects of SSBs on chronic diseases, and cause-specific deaths and disability by age, sex, and country, provide current estimates of the worldwide annual mortality and morbidity related to SSB consumption. In total, our model attributed $>180000$ global deaths in 2010 to SSB consumption, with $72.3 \%$ from diabetes mellitus, $24.2 \%$ from CVD, and $3.5 \%$ from cancers. In addition, $>8.5$ million DALYs were linked to SSB consumption globally. To our knowledge, this investigation represents the first comprehensive, systematic assessment of the worldwide burdens of diabetes mellitus, CVD, and cancers attributable to SSBs.

Our findings demonstrate remarkable geographic heterogeneity in SSB-related mortality and morbidity, with substantial absolute and proportional burdens in Latin America and the Caribbean, and nearly none in East Asia. In Caribbean and Latin American countries, such as Mexico, homemade sugary beverages (eg, "frescas") are often made and consumed in addition to commercially produced SSBs; these high intakes are compounded by some of the highest rates of both obesity and diabetes mellitus in the world. ${ }^{7,8,42}$ The low cost of SSBs, lax regulation of advertising, and poor access to clean drinking water in some Latin American and Caribbean countries could contribute to the high SSB intakes in these regions and are natural targets for policy-driven interventions. ${ }^{43,44}$ Although the global proportion of deaths related to SSB consumption may seem low at first glance at $\sim .2 \%$ of all diabetes mellitus, CVD, and cancer deaths, we found great variability in national and regional burdens, with SSB-attributable proportional mortality in Mexican men aged 20 to 44 years as high as $33 \%$. Importantly, our findings demonstrate that $75 \%$ of deaths and $85 \%$ of DALYs related to sugary beverages occur in low- and middle-income countries, highlighting the need for effective 


\begin{tabular}{|c|c|c|c|c|c|c|}
\hline \multicolumn{7}{|c|}{ Effect Size by Age Group } \\
\hline $25-34$ & $35-44$ & $45-54$ & $55-64$ & $65-74$ & $75-84$ & $85+$ \\
\hline $1.41(1.19-1.66)$ & $1.39(1.18-1.63)$ & $1.32(1.15-1.51)$ & $1.26(1.12-1.41)$ & $1.21(1.10-1.33)$ & $1.16(1.08-1.24)$ & $1.08(1.04-1.12)$ \\
\hline $0.10(0.05-0.15)$ & $0.10(0.05-0.15)$ & $0.10(0.05-0.15)$ & $0.10(0.05-0.15)$ & $0.10(0.05-0.15)$ & $0.10(0.05-0.15)$ & $0.10(0.05-0.15)$ \\
\hline $0.23(0.14-0.32)$ & $0.23(0.14-0.32)$ & $0.23(0.14-0.32)$ & $0.23(0.14-0.32)$ & $0.23(0.14-0.32)$ & $0.23(0.14-0.32)$ & $0.23(0.14-0.32)$ \\
\hline $2.09(1.81-2.40)$ & $1.86(1.67-2.08)$ & $1.67(1.53-1.81)$ & $1.50(1.40-1.60)$ & $1.35(1.28-1.41)$ & $1.21(1.16-1.26)$ & $1.04(0.96-1.12)$ \\
\hline $1.79(1.56-2.06)$ & $1.66(1.51-1.84)$ & $1.55(1.46-1.64)$ & $1.44(1.40-1.48)$ & $1.35(1.32-1.38)$ & $1.26(1.20-1.32)$ & $1.14(1.04-1.26)$ \\
\hline $2.30(0.66-7.95)$ & $2.15(0.80-5.78)$ & $2.02(0.97-4.21)$ & $1.90(1.17-3.07)$ & $1.81(1.45-2.26)$ & $1.63(1.53-1.74)$ & $1.45(1.05-2.01)$ \\
\hline $3.55(2.41-5.23)$ & $3.07(2.28-4.15)$ & $2.66(2.15-3.30)$ & $2.32(2.04-2.63)$ & $2.03(1.95-2.11)$ & $1.70(1.61-1.79)$ & $1.38(1.23-1.56)$ \\
\hline $1.12(1.08-1.16)$ & $1.12(1.08-1.16)$ & $1.12(1.08-1.16)$ & $1.12(1.08-1.16)$ & $1.12(1.08-1.16)$ & $1.12(1.08-1.16)$ & $1.12(1.08-1.16)$ \\
\hline $1.07(1.03-1.12)$ & $1.07(1.03-1.12)$ & $1.07(1.03-1.12)$ & $1.07(1.03-1.12)$ & $1.07(1.03-1.12)$ & $1.07(1.03-1.12)$ & $1.07(1.03-1.12)$ \\
\hline $1.20(1.17-1.24)$ & $1.20(1.17-1.24)$ & $1.20(1.17-1.24)$ & $1.20(1.17-1.24)$ & $1.20(1.17-1.24)$ & $1.20(1.17-1.24)$ & $1.20(1.17-1.24)$ \\
\hline $1.12(1.03-1.23)$ & $1.12(1.03-1.23)$ & $1.12(1.03-1.23)$ & $1.12(1.03-1.23)$ & $1.12(1.03-1.23)$ & $1.12(1.03-1.23)$ & $1.12(1.03-1.23)$ \\
\hline $1.07(0.93-1.23)$ & $1.07(0.93-1.23)$ & $1.07(0.93-1.23)$ & $1.07(0.93-1.23)$ & $1.07(0.93-1.23)$ & $1.07(0.93-1.23)$ & $1.07(0.93-1.23)$ \\
\hline $1.51(1.31-1.74)$ & $1.51(1.31-1.74)$ & $1.51(1.31-1.74)$ & $1.51(1.31-1.74)$ & $1.51(1.31-1.74)$ & $1.51(1.31-1.74)$ & $1.51(1.31-1.74)$ \\
\hline $1.52(1.33-1.74)$ & $1.52(1.33-1.74)$ & $1.52(1.33-1.74)$ & $1.52(1.33-1.74)$ & $1.52(1.33-1.74)$ & $1.52(1.33-1.74)$ & $1.52(1.33-1.74)$ \\
\hline $1.59(1.5-1.68)$ & $1.59(1.5-1.68)$ & $1.59(1.5-1.68)$ & $1.59(1.5-1.68)$ & $1.59(1.5-1.68)$ & $1.59(1.5-1.68)$ & $1.59(1.5-1.68)$ \\
\hline $1.34(1.25-1.43)$ & $1.34(1.25-1.43)$ & $1.34(1.25-1.43)$ & $1.34(1.25-1.43)$ & $1.34(1.25-1.43)$ & $1.34(1.25-1.43)$ & $1.34(1.25-1.43)$ \\
\hline $1.24(1.15-1.34)$ & $1.24(1.15-1.34)$ & $1.24(1.15-1.34)$ & $1.24(1.15-1.34)$ & $1.24(1.15-1.34)$ & $1.24(1.15-1.34)$ & $1.24(1.15-1.34)$ \\
\hline $1.59(1.02-2.47)$ & $1.59(1.02-2.47)$ & $1.59(1.02-2.47)$ & $1.59(1.02-2.47)$ & $1.59(1.02-2.47)$ & $1.59(1.02-2.47)$ & $1.59(1.02-2.47)$ \\
\hline $1.09(0.98-1.2)$ & $1.09(0.98-1.2)$ & $1.09(0.98-1.2)$ & $1.09(0.98-1.2)$ & $1.09(0.98-1.2)$ & $1.09(0.98-1.2)$ & $1.09(0.98-1.2)$ \\
\hline
\end{tabular}

interventions to reduce SSB consumption in not only richer, but also lower-income countries. Furthermore, our model estimates the effect of removing all SSBs independent of any collateral effects that such a change might have. However, it is possible that reduction in SSB intake could additionally influence other dietary behaviors, such as switching to healthier beverages and otherwise improving diet quality. Therefore, the number of deaths and DALYs that could be prevented by reducing SSB consumption globally may be greater than what is estimated by our current model.

The absolute burdens of deaths attributable to SSB consumption are lower than for some risk factors for chronic diseases, including metabolic risk factors, such as BMI, ${ }^{40}$ and other dietary factors, such as excess dietary sodium ${ }^{41}$ and insufficient consumption of fruits and vegetables. ${ }^{45}$ In 2010 , 2.4 million chronic disease deaths were attributable to elevated $\mathrm{BMI},{ }^{40}$ with the BMI-mediated chronic disease mortality attributable to SSB consumption accounting for $\sim 1$ in 12 of those deaths. However, SSBs are but 1 contributor to the obesity epidemic, which is also related to multiple additional factors such as consumption of refined carbohydrates, other dietary sugars, inadequate physical activity, genetics/epigenetics, and psychosocial/environmental factors. In light of this, the number of SSB-related deaths is considerable given that it is only a single component of diet. In 2010, 2.7 million deaths were attributable to elevated intake of dietary sodium ${ }^{41}$ and 4.7 million were attributable to inadequate fruit and vegetable consumption ${ }^{45}$; yet, in comparison with sodium, which is nearly ubiquitous across the food supply, or fruits and vegetables, which represent large and diverse classes of foods, SSBs represent only a single class of beverage. Although substantial increases in global consumption of fruits and vegetables would require major long-term changes to agricultural systems, policies, transport and storage infrastructures, food manufacturing, and sociocultural priorities and norms, SSBs could be decreased without any new investments or technological advances in agriculture, transport, storage, manufacturing, or marketing. 
Table 3. Global And Regional Deaths Related to SSB Consumption in 2010

\begin{tabular}{|c|c|c|c|c|c|c|c|}
\hline & \multicolumn{3}{|c|}{ Population Characteristics } & \multicolumn{4}{|c|}{$\begin{array}{l}\text { Number of Deaths Attributable to SSBs } \\
\qquad(95 \% \text { UI) }\end{array}$} \\
\hline & $\begin{array}{l}\text { Population } \\
\text { (millions) }\end{array}$ & $\begin{array}{l}\text { Mean SSB Intake } \\
\text { (Servings/d) }\end{array}$ & $\begin{array}{l}\text { Mean BMl } \\
\left(\mathrm{kg} / \mathrm{m}^{2}\right)\end{array}$ & $\mathrm{CVD}^{*}$ & Diabetes Mellitus $\dagger$ & Cancersł & Total§ \\
\hline \multicolumn{8}{|l|}{ Globe } \\
\hline \multicolumn{8}{|l|}{ Women } \\
\hline Ages 20-44 & 987 & $0.8(0.3-1.3)$ & $25(23-26)$ & 1299 (891-1725) & 7760 (7152-8337) & $268(186-351)$ & 9327 (8392-10254) \\
\hline Ages 45-64 & 654 & $0.4(0.2-0.6)$ & 27 (25-29) & 4764 (3229-6342) & 27473 (25067-29 900) & $1154(778-1555)$ & 33391 (29786-37 045) \\
\hline Ages $\geq 65$ & 293 & $0.3(0.1-0.5)$ & $26(23-29)$ & 13739 (8905-19263) & 33385 (30527-36 169) & 1854 (1204-2537) & 48978 (41 292-56 966) \\
\hline Women overall & 1930 & $0.5(0.2-0.8)$ & $26(24-28)$ & 19802 (13133-27 332) & 68618 (64 410-72 881) & $3276(2160-4427)$ & 91695 (80923-103015) \\
\hline \multicolumn{8}{|l|}{ Men } \\
\hline Ages 20-44 & 1020 & $0.9(0.4-1.4)$ & $24(23-26)$ & 3045 (2071-4043) & 10291 (9426-11115) & 189 (131-251) & $13525(11887-15214)$ \\
\hline Ages 45-64 & 645 & $0.4(0.2-0.7)$ & $26(24-27)$ & 9846 (6499-13274) & 28713 (26343-31 181) & $1221(839-1626)$ & $39780(34247-45183)$ \\
\hline Ages $\geq 65$ & 231 & $0.3(0.1-0.5)$ & $25(23-27)$ & 12027 (7851-16371) & 25159 (23081-27343) & 1749 (1191-2318) & 38935 (32 988-45331) \\
\hline Men overall & 1890 & $0.6(0.2-0.9)$ & $25(23-27)$ & 24918 (16357-33582) & 64163 (60411-67903) & 3159 (2170-4198) & $92240(80161-105248$ \\
\hline Both sexes overall & 3830 & $0.5(0.2-0.9)$ & $25(23-27)$ & 44680 (29087-60715) & 132652 (125924-139384) & $6449(4333-8637)$ & 183781 (160625-20800؛ \\
\hline \multicolumn{8}{|l|}{ Country income level } \\
\hline High income & 763 & $0.5(0.3-0.6)$ & $27(25-28)$ & 14034 (8571-20249) & 27812 (24 197-31548) & 3056 (1897-4279) & 44901 (34663-56 077) \\
\hline Upper-middle income & 1530 & $0.7(0.3-1.1)$ & $26(24-28)$ & 20727 (13597-28337) & $60118(54138-66061)$ & 2606 (1734-3516) & 83451 (69 469-97914) \\
\hline Lower-middle income & 1210 & $0.6(0.2-1.0)$ & $25(23-27)$ & 8939 (576-1490) & 36886 (33412-40536) & 650 (424-896) & 46475 (34 412-42 921) \\
\hline Low income & 323 & $0.3(0.1-0.5)$ & $22(20-24)$ & $1021(576-1490)$ & 7965 (7395-8532) & $124(67-183)$ & 9109 (8039-10205) \\
\hline \multicolumn{8}{|l|}{ Australia and New Zealand } \\
\hline \multicolumn{8}{|l|}{ Women } \\
\hline Ages 20-44 & 3.6 & $0.4(0.2-0.6)$ & $26(25-27)$ & $1(1-2)$ & $6(5-8)$ & $1(1-2)$ & $9(7-11)$ \\
\hline Ages 45-64 & 3.35 & $0.2(0.1-0.3)$ & $29(28-30)$ & $7(4-10)$ & $26(19-34)$ & $7(4-10)$ & $40(30-51)$ \\
\hline Ages $\geq 65$ & 1.94 & $0.1(0.1-0.2)$ & $28(27-29)$ & $70(38-110)$ & $107(81-132$ & $18(11-25)$ & $195(146-253)$ \\
\hline Overall & 8.89 & $0.2(0.1-0.4)$ & $27(26-28)$ & 79 (45-122) & $139(111-168)$ & $26(15-36)$ & $244(189-310)$ \\
\hline \multicolumn{8}{|l|}{ Men } \\
\hline Ages 20-44 & 3.57 & $0.4(0.2-0.6)$ & $27(26-28)$ & $5(3-7)$ & $10(7-13)$ & $1(0-1)$ & $16(12-20)$ \\
\hline Ages 45-64 & 3.28 & $0.2(0.1-0.3)$ & $29(28-30)$ & $26(16-37)$ & $52(38-66)$ & $8(5-11)$ & $86(65-107)$ \\
\hline Ages $\geq 65$ & 1.64 & $0.1(0.1-0.2)$ & $28(27-29)$ & $70(39-103)$ & $126(98-155)$ & $20(12-28)$ & 215 (163-272) \\
\hline Overall & 8.48 & $0.2(0.1-0.4)$ & $28(27-28)$ & $101(61-146)$ & $188(155-222)$ & $29(17-40)$ & 317 (249-389) \\
\hline Both sexes overall & 17.4 & $0.2(0.1-0.4)$ & $28(27-28)$ & $180(107-263)$ & $327(280-371)$ & $54(33-75)$ & 561 (444-694) \\
\hline \multicolumn{8}{|l|}{ Canada and United States } \\
\hline \multicolumn{8}{|l|}{ Women } \\
\hline Ages 20-44 & 45 & $1.6(1.2-2.0)$ & $26(26-27)$ & $133(77-199)$ & $690(500-850)$ & $40(23-58)$ & $863(660-1057)$ \\
\hline Ages 45-64 & 46.1 & $0.8(0.6-1.0)$ & $29(28-30)$ & 645 (388-936) & 2621 (1921-3363) & $222(132-321)$ & 3488 (2605-4342) \\
\hline Ages $\geq 65$ & 25.6 & $0.6(0.4-0.7)$ & $28(27-29)$ & 2531 (1365-4106) & 4729 (3583-5888) & $437(261-630)$ & 7698 (5816-9888) \\
\hline Overall & 117 & $1.0(0.8-1.2)$ & $28(27-28)$ & $3310(1903-5176)$ & 8040 (6670-9441) & 699 (421-992) & 12049 (9467-14760) \\
\hline \multicolumn{8}{|l|}{ Men } \\
\hline Ages 20-44 & 46.3 & $1.8(1.3-2.2)$ & 27 (27-28) & $378(216-558)$ & 1116 (850-1368) & $33(20-48)$ & $1527(1170-1853)$ \\
\hline Ages 45-64 & 44.2 & $0.9(0.7-1.1)$ & $29(29-30)$ & $1714(1035-2445)$ & 4040 (2899-5119) & 277 (167-399) & 6031 (4617-7501) \\
\hline Ages $\geq 65$ & 19.4 & $0.6(0.5-0.8)$ & $28(27-28)$ & $2365(1334-3569)$ & $4541(3380-5693)$ & $433(257-618)$ & 7339 (5521-9245) \\
\hline Overall & 110 & $1.1(0.8-1.4)$ & $28(27-29)$ & 4457 (2613-6373) & 9697 (8072-11325) & $743(447-1041)$ & $14897(11858-18080)$ \\
\hline Both sexes overall & 226 & $1.1(0.8-1.3)$ & $28(27-28)$ & 7767 (4667-11 383) & 17736 (15540-20 035) & $1443(870-2035)$ & 26946 (21 785-32 008) \\
\hline \multicolumn{8}{|l|}{ East/Central Eurasia } \\
\hline \multicolumn{8}{|l|}{ Women } \\
\hline Ages 20 to 44 & 60.4 & $0.4(0.2-0.6)$ & $24(22-26)$ & $109(68-152)$ & $212(184-240)$ & $17(11-24)$ & $338(279-405)$ \\
\hline Ages 45 to 64 & 55.5 & $0.2(0.1-0.3)$ & $28(26-30)$ & $858(524-1204)$ & 749 (639-855) & $136(81-193)$ & 1743 (1304-2192) \\
\hline Ages 65 and older & 32.5 & $0.1(0.1-0.2)$ & $28(24-31)$ & 4093 (2397-5798) & $1301(1154-1451$ & $252(152-358)$ & 5647 (3802-7521) \\
\hline Overall & 148 & $0.2(0.1-0.4)$ & $26(24-29)$ & 5061 (2994-7115) & 2262 (2066-2472) & 405 (244-574) & 7728 (5397-10114) \\
\hline \multicolumn{8}{|l|}{ Men } \\
\hline Ages 20 to 44 & 59.7 & $0.4(0.2-0.6)$ & $25(24-27)$ & 496 (305-697) & 340 (299-381) & $15(9-20)$ & 850 (642-1081) \\
\hline
\end{tabular}




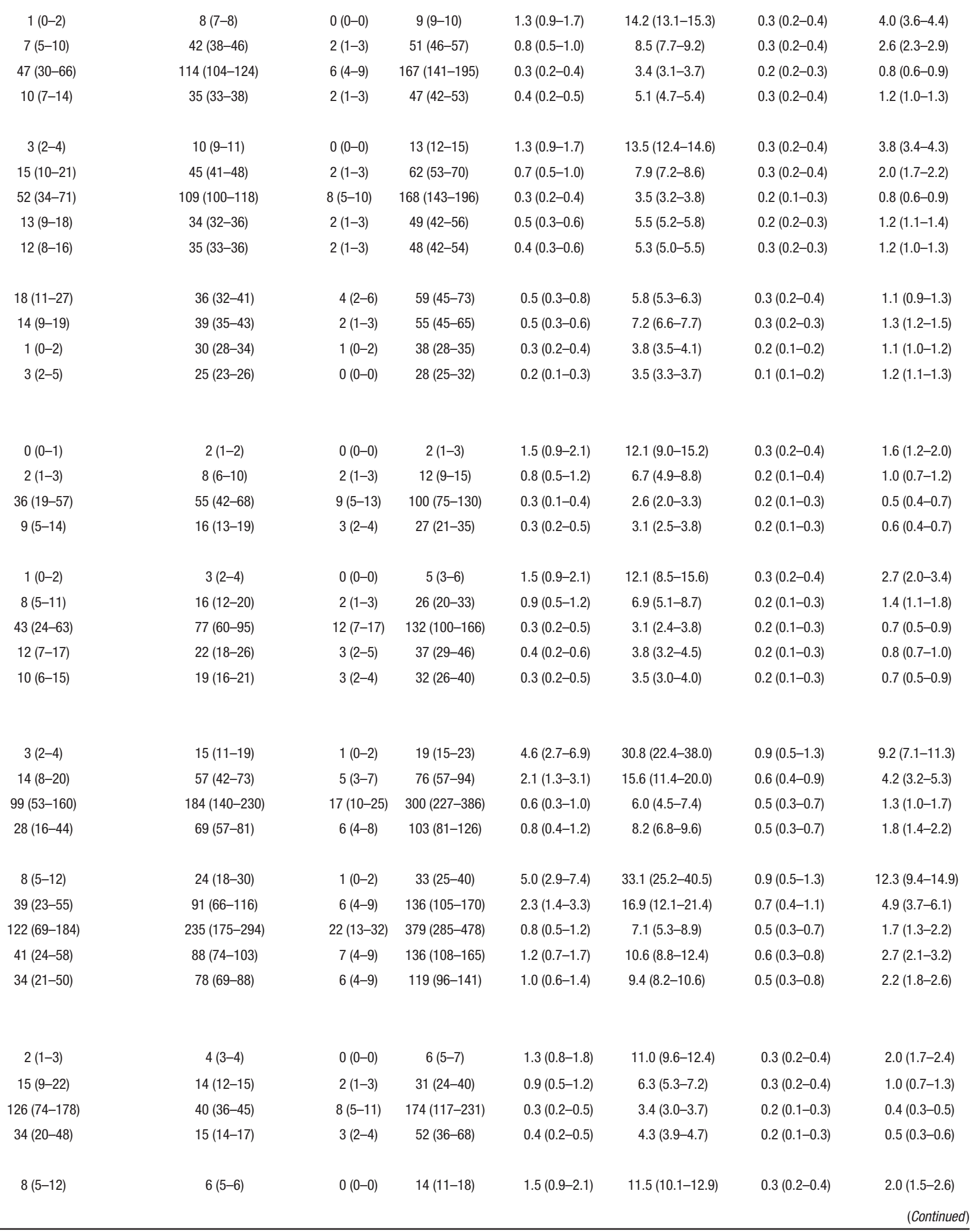


Table 3. Continued

\begin{tabular}{|c|c|c|c|c|c|c|c|}
\hline & \multicolumn{3}{|c|}{ Population Characteristics } & \multicolumn{4}{|c|}{$\begin{array}{l}\text { Number of Deaths Attributable to SSBs } \\
\qquad(95 \% \mathrm{UI})\end{array}$} \\
\hline & $\begin{array}{l}\text { Population } \\
\text { (millions) }\end{array}$ & $\begin{array}{l}\text { Mean SSB Intake } \\
\text { (Servings/d) }\end{array}$ & $\begin{array}{l}\text { Mean BMl } \\
\left(\mathrm{kg} / \mathrm{m}^{2}\right)\end{array}$ & $\mathrm{CVD}^{\star}$ & Diabetes Mellitus† & Cancersł & Total§ \\
\hline Ages 45 to 64 & 47.3 & $0.2(0.1-0.3)$ & $27(25-29)$ & 2410 (1459-3389) & $846(752-942)$ & $161(98-228)$ & 3417 (2352-4535) \\
\hline Ages 65 and older & 17.6 & $0.1(0.1-0.2)$ & $26(24-29)$ & 3009 (1842-4216) & 759 (686-834) & $200(122-278)$ & 3969 (2676-5319) \\
\hline Overall & 125 & $0.3(0.1-0.4)$ & $26(24-28)$ & 5915 (3625-8342) & 1945 (1798-2093) & $376(231-527)$ & 8236 (5675-10 849) \\
\hline Both sexes overall & 273 & $0.2(0.1-0.4)$ & $26(24-29)$ & $10976(6591-15346)$ & 4206 (3913-4508) & 781 (475-1098) & 15964 (11097-20903) \\
\hline \multicolumn{8}{|l|}{ East and Southeast Asia } \\
\hline \multicolumn{8}{|l|}{ Women } \\
\hline Ages 20-44 & 341 & $0.9(0.4-1.3)$ & $25(23-26)$ & $162(96-234)$ & 1849 (1549-2151) & $44(27-62)$ & 2055 (1735-2378) \\
\hline Ages 45-64 & 238 & $0.4(0.2-0.7)$ & $27(25-28)$ & $679(436-931)$ & 7484 (6267-8654) & $194(116-278)$ & 8356 (7044-9610) \\
\hline Ages $\geq 65$ & 101 & $0.3(0.1-0.5)$ & $25(22-27)$ & 1565 (833-2348) & 8651 (7223-10117) & $275(143-414)$ & 10491 (8745-12412) \\
\hline Overall & 680 & $0.5(0.2-0.9)$ & $25(23-27)$ & 2406 (1380-3492) & $17983(16005-19920)$ & $513(285-752)$ & 20902 (18392-23602) \\
\hline \multicolumn{8}{|l|}{ Men } \\
\hline Ages 20-44 & 352 & $0.9(0.4-1.4)$ & $24(23-25)$ & $424(244-613)$ & 2337 (1966-2717) & $38(20-56)$ & 2799 (2344-3253) \\
\hline Ages 45-64 & 243 & $0.5(0.2-0.7)$ & $25(24-27)$ & 1448 (890-2028) & 7275 (6250-8331) & $296(161-449)$ & 9019 (7723-10305) \\
\hline Ages $\geq 65$ & 84.6 & $0.3(0.2-0.5)$ & $24(22-26)$ & 1528 (793-2315) & $4982(4132-5857)$ & 385 (194-579) & 6895 (5496-8280) \\
\hline Overall & 680 & $0.6(0.3-0.9)$ & $24(23-26)$ & 3400 (1920-4949) & 14594 (13237-15992) & $719(384-1073)$ & $18713(16178-21266)$ \\
\hline Both sexes overall & 1360 & $0.6(0.3-0.9)$ & $25(23-27)$ & 5807 (3314-8421) & 32577 (30 041-35 102) & $1232(670-1807)$ & 39615 (35154-44 109) \\
\hline \multicolumn{8}{|c|}{ Latin America and Caribbean } \\
\hline \multicolumn{8}{|l|}{ Women } \\
\hline Ages 20-44 & 88.1 & $1.7(0.7-2.7)$ & $26(25-28)$ & $290(182-402)$ & 1760 (1513-1958) & $71(43-100)$ & 2121 (1810-2419) \\
\hline Ages 45-64 & 54.2 & $0.8(0.3-1.4)$ & $29(27-31)$ & $947(572-1313)$ & 7409 (6092-8614) & $249(147-354)$ & 8605 (7111-10031) \\
\hline \multirow[t]{2}{*}{ Ages $\geq 65$} & 22.7 & $0.6(0.3-1.0)$ & $27(24-30)$ & $2214(1379-3141)$ & 9992 (8510-11441) & $336(205-468)$ & $12541(10455-14$ \\
\hline & & & & & & & 628) \\
\hline Overall & 165 & $1.1(0.4-1.7)$ & $27(25-30)$ & 3452 (2120-4845) & 19161 (16982-21159) & $655(403-919)$ & $23268(19980-26370)$ \\
\hline \multicolumn{8}{|l|}{ Men } \\
\hline Ages 20-44 & 85.6 & $1.8(0.7-2.9)$ & $25(24-27)$ & $648(408-905)$ & 2650 (2255-2957) & $42(25-59)$ & 3340 (2813-3868) \\
\hline Ages 45-64 & 50.3 & $0.9(0.3-1.5)$ & 27 (25-28) & 1798 (1088-2552) & 8569 (6955-9929) & $181(111-254)$ & 10549 (8476-12410) \\
\hline Ages $\geq 65$ & 17.9 & $0.7(0.3-1.1)$ & $25(23-27)$ & 2116 (1349-2919) & 8092 (6758-9376) & $221(145-305)$ & 10429 (8687-12186) \\
\hline Overall & 154 & $1.2(0.4-1.9)$ & $26(24-27)$ & $4562(2872-6297)$ & 19311 (17115-21458) & $445(279-613)$ & 24318 (20736-27976) \\
\hline Both sexes overall & 319 & $1.1(0.4-1.8)$ & $26(24-29)$ & 8013 (4992-11 191) & 38472 (35 106-41 892) & $1100(682-1524)$ & $47585(41061-53845)$ \\
\hline \multicolumn{8}{|c|}{ North Africa and Middle East } \\
\hline \multicolumn{8}{|l|}{ Women } \\
\hline Ages 20-44 & 66.1 & $0.6(0.2-1.1)$ & $27(26-28)$ & $234(142-330)$ & $515(456-570)$ & 27 (16-38) & $776(651-926)$ \\
\hline Ages 45-64 & 32.8 & $0.3(0.1-0.6)$ & $30(29-32)$ & $563(341-801)$ & $1363(1202-1528)$ & $52(32-74)$ & 1979 (1627-2321) \\
\hline Ages $\geq 65$ & 11.2 & $0.3(0.1-0.4)$ & $28(26-31)$ & $995(600-1400)$ & 2067 (1811-2317) & $43(26-61)$ & 3106 (2518-3694) \\
\hline Overall & 110 & $0.4(0.1-0.7)$ & $28(27-30)$ & 1793 (1087-2564) & 3945 (3585-4285) & $123(75-172)$ & $5861(4840-6860)$ \\
\hline \multicolumn{8}{|l|}{ Men } \\
\hline Ages 20-44 & 71.6 & $0.7(0.2-1.1)$ & $26(25-27)$ & $496(308-693)$ & $636(567-701)$ & $14(9-19)$ & $1146(920-1377)$ \\
\hline Ages 45-64 & 33.5 & $0.3(0.1-0.6)$ & $28(26-29)$ & $1006(606-1409)$ & 1485 (1292-1672) & $32(19-45)$ & $2523(1984-3053)$ \\
\hline Ages $\geq 65$ & 9.67 & $0.3(0.1-0.4)$ & $26(25-28)$ & $1005(626-1400)$ & 1668 (1476-1854) & $34(22-46)$ & 2707 (2204-3249) \\
\hline Overall & 115 & $0.4(0.2-0.7)$ & $27(25-28)$ & 2507 (1551-3490) & $3789(3482-4110)$ & $79(50-110)$ & $6376(5165-7616)$ \\
\hline Both sexes overall & 225 & $0.4(0.2-0.7)$ & $28(26-29)$ & $4300(2640-5986)$ & 7734 (7190-8293) & $202(125-283)$ & $12236(10059-14421)$ \\
\hline \multicolumn{8}{|l|}{ South Asia } \\
\hline \multicolumn{8}{|l|}{ Women } \\
\hline Ages 20-44 & 224 & $0.3(0.1-0.5)$ & $22(20-23)$ & $216(112-333)$ & $1260(942-1574)$ & $36(18-55)$ & $1512(1145-1851)$ \\
\hline Ages 45-64 & 122 & $0.2(0.0-0.3)$ & $22(20-24)$ & $621(317-945)$ & 4639 (3378-5965) & $118(61-184)$ & 5378 (4016-6767) \\
\hline Ages $\geq 65$ & 40.1 & $0.1(0.0-0.2)$ & $21(18-23)$ & $126(64-193)$ & 887 (634-1160) & $12(6-19)$ & 1026 (763-1319) \\
\hline Overall & 385 & $0.2(0.1-0.3)$ & $21(19-23)$ & 963 (498-1467) & 6787 (5461-8192) & 167 (85-253) & 7916 (6458-9589) \\
\hline \multicolumn{8}{|l|}{ Men } \\
\hline Ages 20-44 & 238 & $0.4(0.1-0.6)$ & $21(20-23)$ & $355(177-554)$ & 1570 (1033-2111) & $20(10-31)$ & 1945 (1361-2541) \\
\hline Ages 45-64 & 126 & $0.2(0.0-0.3)$ & $22(20-23)$ & 608 (308-921) & 2858 (1791-3897) & $56(28-91)$ & $3522(2410-4585)$ \\
\hline
\end{tabular}




\begin{tabular}{|c|c|c|c|c|c|c|c|}
\hline \multicolumn{4}{|c|}{ Deaths per Million Adults Attributable to SSBs (95\% UI) } & \multicolumn{4}{|c|}{ Proportion of Deaths Attributable To SSBs ( $95 \%$ UI) } \\
\hline $\mathrm{CVD}^{*}$ & Diabetes Mellitus $†$ & Cancersł & Total§ & $\mathrm{CVD}^{*}$ & Diabetes Mellitus† & Cancersł & Total§ \\
\hline $51(31-72)$ & $18(16-20)$ & $3(2-5)$ & $72(50-96)$ & $0.9(0.5-1.2)$ & $6.4(5.7-7.1)$ & $0.3(0.2-0.4)$ & $1.0(0.7-1.3)$ \\
\hline $171(105-240)$ & $43(39-47)$ & $11(7-16)$ & $226(152-302)$ & $0.4(0.2-0.6)$ & $3.6(3.3-4.0)$ & $0.2(0.1-0.2)$ & $0.5(0.3-0.6)$ \\
\hline $47(29-67)$ & $16(14-17)$ & $3(2-4)$ & $66(46-87)$ & $0.6(0.3-0.8)$ & $5.2(4.8-5.6)$ & $0.2(0.1-0.3)$ & $0.6(0.4-0.9)$ \\
\hline $40(24-56)$ & $15(14-17)$ & $3(2-4)$ & $58(41-77)$ & $0.5(0.3-0.6)$ & $4.7(4.4-5.0)$ & $0.2(0.1-0.3)$ & $0.5(0.4-0.7)$ \\
\hline $0(0-0)$ & $5(4-6)$ & $0(0-0)$ & $6(5-7)$ & $0.8(0.5-1.1)$ & $11.8(9.8-13.7)$ & $0.2(0.1-0.2)$ & $3.2(2.7-3.7)$ \\
\hline $3(2-4)$ & $31(26-36)$ & $1(0-2)$ & $35(30-40)$ & $0.5(0.3-0.6)$ & $6.7(5.6-7.8)$ & $0.1(0.1-0.2)$ & $2.3(1.9-2.6)$ \\
\hline $16(8-23)$ & $86(72-100)$ & $3(1-4)$ & $104(87-123)$ & $0.1(0.1-0.2)$ & $2.9(2.4-3.4)$ & $0.1(0.1-0.2)$ & $0.6(0.5-0.7)$ \\
\hline $1(0-2)$ & $7(6-8)$ & $0(0-0)$ & $8(7-9)$ & $0.7(0.4-1.1)$ & $11.1(9.4-13.0)$ & $0.1(0.1-0.2)$ & $2.9(2.4-3.4)$ \\
\hline $6(4-8)$ & $30(26-34)$ & $1(0-2)$ & 37 (32-42) & $0.4(0.3-0.6)$ & $6.8(5.9-7.8)$ & $0.2(0.1-0.3)$ & $1.6(1.3-1.8)$ \\
\hline $18(9-27)$ & 59 (49-69) & $5(2-7)$ & $82(65-98)$ & $0.1(0.1-0.2)$ & $2.5(2.0-2.9)$ & $0.1(0.1-0.2)$ & $0.4(0.4-0.5)$ \\
\hline $5(3-7)$ & 21 (19-24) & $1(0-2)$ & $28(24-31)$ & $0.2(0.1-0.3)$ & $4.4(4.0-4.9)$ & $0.1(0.1-0.2)$ & $0.8(0.7-0.9)$ \\
\hline $4(2-6)$ & $24(22-26)$ & $1(0-2)$ & $29(26-32)$ & $0.2(0.1-0.3)$ & $4.3(4.0-4.6)$ & $0.1(0.1-0.2)$ & $0.9(0.8-1.0)$ \\
\hline $3(2-5)$ & $20(17-22)$ & $1(0-2)$ & $24(21-27)$ & $3.9(2.4-5.4)$ & $30.4(26.2-33.9)$ & $1.0(0.6-1.4)$ & $10.9(9.3-12.4)$ \\
\hline $17(11-24)$ & 137 (112-159) & $5(3-7)$ & $159(131-185)$ & $2.0(1.2-2.8)$ & $17.1(14.1-19.9)$ & $0.8(0.5-1.1)$ & $7.7(6.4-9.0)$ \\
\hline $98(61-138)$ & $440(375-504)$ & $15(9-21)$ & $552(460-644)$ & $0.8(0.5-1.1)$ & $7.3(6.2-8.4)$ & $0.6(0.4-0.8)$ & $2.7(2.3-3.2)$ \\
\hline $8(5-11)$ & $31(26-35)$ & $0(0-2)$ & 39 (33-45) & $4.4(2.8-6.1)$ & $33.4(28.4-37.2)$ & $0.7(0.4-1.0)$ & $13.9(11.7-16.1)$ \\
\hline $36(22-51)$ & $170(138-197)$ & $4(2-5)$ & $210(169-247)$ & $2.1(1.3-3.0)$ & $18.5(15.0-21.4)$ & $0.7(0.4-1.0)$ & $7.5(6.0-8.8)$ \\
\hline $118(75-163)$ & $452(377-524)$ & $12(8-17)$ & $582(485-681)$ & $0.8(0.5-1.1)$ & $8.5(7.1-9.9)$ & $0.4(0.3-0.6)$ & $2.7(2.3-3.2)$ \\
\hline 30 (19-41) & $126(111-140)$ & $3(2-4)$ & $158(135-182)$ & $1.2(0.8-1.7)$ & $12.9(11.5-14.4)$ & $0.5(0.3-0.7)$ & $4.4(3.8-5.1)$ \\
\hline $25(16-35)$ & $121(110-131)$ & $3(2-5)$ & 149 (129-169) & $1.1(0.7-1.6)$ & $11.5(10.5-12.5)$ & $0.6(0.4-0.9)$ & $4.2(3.6-4.7)$ \\
\hline $4(2-5)$ & $8(7-9)$ & $0(0-1)$ & $12(10-14)$ & $2.0(1.2-2.8)$ & $14.4(12.8-16.0)$ & $0.4(0.3-0.6)$ & $3.5(2.9-4.1)$ \\
\hline 17 (10-24) & $42(37-47)$ & $2(1-3)$ & $60(50-71)$ & $1.1(0.7-1.6)$ & $7.9(7.0-8.9)$ & $0.4(0.2-0.5)$ & $2.4(2.0-2.8)$ \\
\hline $89(54-125)$ & 185 (162-208) & $4(2-6)$ & $278(226-331)$ & $0.5(0.3-0.7)$ & $4.0(3.5-4.5)$ & $0.3(0.2-0.5)$ & $1.2(1.0-1.4)$ \\
\hline $16(10-23)$ & 36 (33-39) & $1(0-2)$ & $53(44-62)$ & $0.7(0.4-1.0)$ & $5.4(4.9-5.9)$ & $0.4(0.2-0.5)$ & $1.6(1.3-1.9)$ \\
\hline $7(4-10)$ & $9(8-10)$ & $0(0-0)$ & $16(13-19)$ & $1.9(1.2-2.7)$ & $15.0(13.4-16.6)$ & $0.3(0.2-0.4)$ & $3.4(2.8-4.1)$ \\
\hline $30(18-42)$ & $44(39-50)$ & $1(1-1)$ & 75 (59-91) & $1.1(0.6-1.5)$ & $8.2(7.1-9.2)$ & $0.3(0.2-0.4)$ & $2.1(1.6-2.5)$ \\
\hline $104(65-145)$ & $172(153-192)$ & $3(2-5)$ & $280(228-336)$ & $0.5(0.3-0.7)$ & $4.2(3.7-4.7)$ & $0.2(0.1-0.3)$ & $1.0(0.8-1.2)$ \\
\hline $22(14-30)$ & $33(30-36)$ & $1(0-2)$ & $56(45-66)$ & $0.8(0.5-1.0)$ & $6.1(5.6-6.6)$ & $0.2(0.1-0.3)$ & $1.5(1.2-1.8)$ \\
\hline $19(12-27)$ & $34(32-37)$ & $1(0-2)$ & $54(45-64)$ & $0.7(0.4-1.0)$ & $5.7(5.3-6.2)$ & $0.3(0.2-0.4)$ & $1.6(1.3-1.8)$ \\
\hline $1(0-2)$ & $6(4-7)$ & $0(0-0)$ & $7(5-8)$ & $0.6(0.3-0.9)$ & $9.0(6.7-11.2)$ & $0.2(0.1-0.3)$ & $2.2(1.7-2.7)$ \\
\hline $5(3-8)$ & 38 (28-49) & $1(0-2)$ & $44(33-56)$ & $0.4(0.2-0.6)$ & $5.8(4.2-7.5)$ & $0.2(0.1-0.3)$ & $1.8(1.3-2.3)$ \\
\hline $3(2-5)$ & $22(16-29)$ & $0(0-0)$ & $26(19-33)$ & $0.0(0.0-0.0)$ & $0.5(0.4-0.6)$ & $0.0(0.0-0.0)$ & $0.1(0.1-0.2)$ \\
\hline $2(1-4)$ & $18(14-21)$ & $0(0-1)$ & $21(17-25)$ & $0.1(0.1-0.2)$ & $2.5(2.0-3.0)$ & $0.1(0.1-0.2)$ & $0.7(0.6-0.9)$ \\
\hline
\end{tabular}


Table 3. Continued

\begin{tabular}{|c|c|c|c|c|c|c|c|}
\hline & \multicolumn{3}{|c|}{ Population Characteristics } & \multicolumn{4}{|c|}{$\begin{array}{l}\text { Number of Deaths Attributable to SSBs } \\
(95 \% \text { UI) }\end{array}$} \\
\hline & $\begin{array}{c}\text { Population } \\
\text { (millions) }\end{array}$ & $\begin{array}{l}\text { Mean SSB Intake } \\
\text { (Servings/d) }\end{array}$ & $\begin{array}{c}\text { Mean BMl } \\
\left(\mathrm{kg} / \mathrm{m}^{2}\right)\end{array}$ & $\mathrm{CVD}^{*}$ & Diabetes Mellitus† & Cancersł & Total§ \\
\hline Ages $\geq 65$ & 36.5 & $0.1(0.0-0.2)$ & $21(19-23)$ & $89(47-136)$ & $590(379-806)$ & $6(3-10)$ & 685 (463-914) \\
\hline Overall & 401 & $0.2(0.1-0.4)$ & $21(19-23)$ & $1053(543-1601)$ & $5017(3900-6227)$ & $83(43-128)$ & 6152 (4806-7478) \\
\hline Both sexes overall & 786 & $0.2(0.1-0.4)$ & $21(19-23)$ & 2015 (1042-3051) & $11803(10025-13663)$ & $250(130-376)$ & $14068(11790-16456)$ \\
\hline \multicolumn{8}{|l|}{ Sub-Saharan Africa } \\
\hline \multicolumn{8}{|l|}{ Women } \\
\hline Ages 20-44 & 102 & $0.7(0.2-1.2)$ & $23(22-25)$ & $133(89-181)$ & 1399 (1277-1516) & $17(12-23)$ & 1549 (1399-1700) \\
\hline Ages 45-64 & 46 & $0.3(0.1-0.6)$ & $25(23-26)$ & $294(200-396)$ & 2818 (2516-3113) & $51(34-68)$ & 3163 (2814-3509) \\
\hline Ages $\geq 65$ & 15.1 & $0.2(0.1-0.4)$ & $23(20-26)$ & $420(285-565)$ & 3060 (2697-3398) & $42(29-56)$ & 3522 (3085-3938) \\
\hline Overall & 163 & $0.4(0.1-0.8)$ & $24(21-26)$ & 847 (573-1136) & 7277 (6761-7739) & $110(74-148)$ & 8234 (7514-8915) \\
\hline \multicolumn{8}{|l|}{ Men } \\
\hline Ages 20-44 & 103 & $0.8(0.2-1.3)$ & $22(20-24)$ & $148(90-211)$ & 1453 (1335-1574) & $13(8-18)$ & 1613 (1463-1777) \\
\hline Ages $45-64$ & 42.5 & $0.4(0.1-0.6)$ & $23(21-25)$ & $288(187-393)$ & 2765 (2486-3059) & $48(32-65)$ & 3101 (2767-3452) \\
\hline Ages $\geq 65$ & 12.3 & $0.3(0.1-0.5)$ & $22(20-25)$ & $266(170-366)$ & 2215 (2001-2433) & $39(26-52)$ & $2519(2252-2790)$ \\
\hline Overall & 158 & $0.5(0.1-0.8)$ & $22(20-24)$ & $702(455-970)$ & 6432 (6035-6837) & $100(67-135)$ & $7234(6625-7840)$ \\
\hline Both sexes overall & 320 & $0.5(0.1-0.8)$ & $23(21-25)$ & 1549 (1043-2096) & 13709 (12984-14396) & $210(141-281)$ & 15468 (14 295-16610) \\
\hline \multicolumn{8}{|l|}{ Western Europe } \\
\hline \multicolumn{8}{|l|}{ Women } \\
\hline Ages 20-44 & 57.2 & $0.6(0.4-0.7)$ & $24(23-26)$ & $19(13-26)$ & $71(63-78)$ & $15(9-20)$ & $104(88-120)$ \\
\hline Ages 45-64 & 55.9 & $0.2(0.2-0.3)$ & $28(26-30)$ & $149(90-209)$ & $363(317-408)$ & $125(76-176)$ & 638 (502-778) \\
\hline Ages $\geq 65$ & 42.9 & $0.2(0.1-0.2)$ & $28(25-30)$ & 1724 (1014-2488) & 2591 (2325-2877) & $438(260-623)$ & 4752 (3769-5788) \\
\hline Overall & 156 & $0.4(0.3-0.5)$ & $26(24-28)$ & 1892 (1115-2722) & 3025 (2753-3317) & $578(348-823)$ & 5494 (4364-6646) \\
\hline \multicolumn{8}{|l|}{ Men } \\
\hline Ages 20-44 & 58.4 & $0.7(0.5-0.9)$ & $26(25-27)$ & $96(59-134)$ & 180 (156-202) & $14(8-19)$ & $289(232-345)$ \\
\hline Ages 45-64 & 54.8 & $0.3(0.2-0.3)$ & $28(27-30)$ & 547 (332-768) & $824(721-934)$ & $161(97-226)$ & $1532(1191-1881)$ \\
\hline Ages $\geq 65$ & 31.9 & $0.2(0.1-0.3)$ & $28(26-29)$ & 1579 (941-2204) & 2187 (1939-2429) & $411(249-579)$ & $4177(3222-5139)$ \\
\hline Overall & 145 & $0.4(0.3-0.5)$ & $27(26-28)$ & $2222(1337-3116)$ & 3191 (2871-3483) & 586 (354-823) & 5998 (4645-7365) \\
\hline Both sexes overall & 301 & $0.4(0.3-0.5)$ & $27(25-28)$ & $4114(2460-5750)$ & $6215(5768-6701)$ & $1163(703-1643)$ & 11493 (9044-13926) \\
\hline
\end{tabular}

Countries are grouped into regions as follows: Australia/New Zealand - Australia, New Zealand; East/Central Eurasia - Albania, Armenia, Azerbaijan, Bulgaria, Bosnia and Herzegovina, Belarus, Czech Republic, Estonia, Georgia, Croatia, Hungary, Kazakhstan, Kyrgyzstan, Lithuania, Latvia, Moldova, Macedonia, Montenegro, Mongolia, Poland, Romania, Russian Federation, Serbia, Slovakia, Slovenia, Tajikistan, Turkmenistan, Ukraine, Uzbekistan; East/Southeast Asia - Brunei Darussalam, China, Fiji, Micronesia, Indonesia, Japan, Cambodia, Kiribati, Republic of Korea, Lao People's Democratic Republic, Sri Lanka, Maldives, Marshall Islands, Myanmar, Malaysia, Philippines, Papua New Guinea, Democratic People's Republic of Korea, Singapore, Solomon Islands, Thailand, Timor-Leste, Tonga, Taiwan, Viet Nam, Vanuatu, Samoa; Latin America/Caribbean Argentina, Antigua and Barbuda, Bahamas, Belize, Bolivia, Brazil, Barbados, Chile, Colombia, Costa Rica, Cuba, Dominica, Dominican Republic, Ecuador, Grenada, Guatemala, Guyana, Honduras, Haiti, Jamaica, Saint Lucia, Mexico, Nicaragua, Panama, Peru, Paraguay, El Salvador, Suriname, Trinidad and Tobago, Uruguay, Saint Vincent and the Grenadines, Venezuela; North Africa/Middle East - United Arab Emirates, Bahrain, Algeria, Egypt, Iran, Iraq, Jordan, Kuwait, Lebanon, Libyan Arab Jamahiriya, Morocco, Oman, Occupied Palestinian Territory, Qatar, Saudi Arabia, Syrian Arab Republic, Tunisia, Turkey, Yemen; South Asia - Afghanistan, Bangladesh, Bhutan, India, Nepal, Pakistan; SubSaharan Africa - Angola, Burundi, Benin, Burkina Faso, Botswana, Central African Republic, Côte d'lvoire, Cameroon, Democratic Republic of the Congo, Congo, Comoros, Cape Verde, Djibouti, Eritrea, Ethiopia, Gabon, Ghana, Guinea, Gambia, Guinea-Bissau, Equatorial Guinea, Kenya, Liberia, Lesotho, Madagascar, Mali, Mozambique, Mauritania, Mauritius, Malawi, Namibia, Niger, Nigeria, Rwanda, Sudan, Senegal, Sierra Leone, Somalia, São Tomé and Príncipe, Swaziland, Seychelles, Chad, Togo, United Republic of Tanzania, Uganda, South Africa, Zambia, Zimbabwe; Western Europe - Andorra, Austria, Belgium, Switzerland, Cyprus, Germany, Denmark, Spain, Finland, France, United Kingdom, Greece, Ireland, Iceland, Israel, Italy, Luxembourg, Malta, Netherlands, Norway, Portugal, Sweden. BMI indicates body mass index; CVD, cardiovascular disease; SSB, sugar-sweetened beverages; and UI, uncertainty intervals.

The burdens attributable to SSB are also relatively unique because of their predominant proportional impact on the young. Throughout much of the world, intakes at younger ages are far higher than later in life. Consequently, the proportional mortality attributable to SSBs is remarkably high among younger adults, exceeding 1 in 10 of all diabetes mellitus- and obesityrelated deaths in nearly every region of the world. Younger adults also constitute the largest proportion of the workforce in most countries, producing tremendous economic losses related to SSB intakes in these age groups. ${ }^{47}$ Our findings suggest that $\sim 1$ in 20 diabetes mellitus-related DALYs in adults $<44$ years of age are attributable to SSB intake, highlighting the considerable social and financial impacts of SSB consumption from not only healthcare costs of managing diabetes mellitus, but also losses of wages and productivity.

Although we demonstrated an inverse age effect in proportional disease burdens attributable to SSBs, it remains to be seen whether this pattern represents an effect of aging (ie, owing to people decreasing their intakes as they get older) or a birth cohort or generational effect (ie, owing to more recent 


\begin{tabular}{|c|c|c|c|c|c|c|c|}
\hline \multicolumn{4}{|c|}{ Deaths per Million Adults Attributable to SSBs (95\% UI) } & \multicolumn{4}{|c|}{ Proportion of Deaths Attributable To SSBs (95\% UI) } \\
\hline CVD* $^{*}$ & Diabetes Mellitus $†$ & Cancersł & Total§ & CVD* $^{*}$ & Diabetes Mellitus $†$ & Cancersł & Total§ \\
\hline $2(1-4)$ & $16(10-22)$ & $0(0-0)$ & $19(13-25)$ & $0.0(0.0-0.0)$ & $0.3(0.2-0.5)$ & $0.0(0.0-0.0)$ & $0.1(0.1-0.1)$ \\
\hline $3(1-4)$ & $13(10-16)$ & $0(0-0)$ & $15(12-19)$ & $0.1(0.1-0.2)$ & $1.7(1.3-2.1)$ & $0.1(0.0-0.1)$ & $0.4(0.3-0.5)$ \\
\hline $3(1-4)$ & $15(13-17)$ & $0(0-0)$ & $18(15-21)$ & $0.1(0.1-0.2)$ & $2.0(1.7-2.4)$ & $0.1(0.1-0.2)$ & $0.6(0.5-0.6)$ \\
\hline $1(1-2)$ & $14(13-15)$ & $0(0-0)$ & $15(14-17)$ & $1.2(0.8-1.6)$ & $13.2(12.1-14.3)$ & $0.3(0.2-0.4)$ & $6.1(5.5-6.6)$ \\
\hline $6(4-9)$ & $61(55-68)$ & $1(0-2)$ & $69(61-76)$ & $0.6(0.4-0.8)$ & $7.3(6.5-8.1)$ & $0.3(0.2-0.3)$ & $3.3(2.9-3.6)$ \\
\hline $28(19-37)$ & $203(179-225)$ & $3(2-4)$ & $233(204-261)$ & $0.3(0.2-0.4)$ & $3.8(3.3-4.2)$ & $0.2(0.2-0.3)$ & $1.5(1.3-1.7)$ \\
\hline $5(4-7)$ & $45(42-48)$ & $1(0-2)$ & $51(46-55)$ & $0.4(0.3-0.5)$ & $5.6(5.2-5.9)$ & $0.2(0.2-0.3)$ & $2.3(2.1-2.5)$ \\
\hline $1(0-2)$ & $14(13-15)$ & $0(0-0)$ & $16(14-17)$ & $1.0(0.6-1.4)$ & $12.6(11.6-13.7)$ & $0.3(0.2-0.5)$ & $6.1(5.5-6.7)$ \\
\hline $7(4-9)$ & $65(59-72)$ & $1(1-2)$ & $73(65-81)$ & $0.5(0.3-0.7)$ & $7.1(6.3-7.8)$ & $0.4(0.2-0.5)$ & $3.2(2.8-3.5)$ \\
\hline $22(14-30)$ & $180(163-198)$ & $3(2-4)$ & $205(183-227)$ & $0.2(0.2-0.3)$ & $4.0(3.6-4.4)$ & $0.3(0.2-0.4)$ & $1.5(1.4-1.7)$ \\
\hline $4(3-6)$ & $41(38-43)$ & $1(0-1)$ & $46(42-50)$ & $0.4(0.2-0.5)$ & $6.0(5.7-6.4)$ & $0.3(0.2-0.4)$ & $2.5(2.3-2.7)$ \\
\hline $5(3-7)$ & $43(41-45)$ & $1(0-2)$ & $48(45-52)$ & $0.4(0.3-0.5)$ & $5.8(5.5-6.1)$ & $0.3(0.2-0.4)$ & $2.4(2.2-2.6)$ \\
\hline $0(0-0)$ & $1(0-2)$ & $0(0-0)$ & $2(1-3)$ & $1.2(0.8-1.6)$ & $12.2(10.8-13.5)$ & $0.3(0.2-0.3)$ & $1.2(1.0-1.4)$ \\
\hline $3(2-4)$ & $6(6-7)$ & $2(1-3)$ & $11(9-14)$ & $0.9(0.5-1.2)$ & $6.6(5.8-7.4)$ & $0.3(0.2-0.4)$ & $0.9(0.7-1.1)$ \\
\hline $40(24-58)$ & $60(54-67)$ & $10(6-15)$ & $111(88-135)$ & $0.3(0.2-0.4)$ & $2.5(2.2-2.8)$ & $0.2(0.1-0.3)$ & $0.5(0.4-0.7)$ \\
\hline $12(7-17)$ & $19(18-21)$ & $4(2-5)$ & $35(28-43)$ & $0.3(0.2-0.5)$ & $2.7(2.5-3.0)$ & $0.2(0.1-0.3)$ & $0.6(0.5-0.7)$ \\
\hline $2(1-2)$ & $3(3-3)$ & $0(0-0)$ & $5(4-6)$ & $1.6(1.0-2.2)$ & $13.5(11.8-15.2)$ & $0.3(0.2-0.5)$ & $2.8(2.3-3.4)$ \\
\hline $10(6-14)$ & $15(13-17)$ & $3(2-4)$ & $28(22-34)$ & $0.9(0.5-1.3)$ & $6.9(6.1-7.8)$ & $0.3(0.2-0.4)$ & $1.3(1.0-1.6)$ \\
\hline $49(29-69)$ & $68(61-76)$ & $13(8-18)$ & $131(101-161)$ & $0.4(0.2-0.5)$ & $3.1(2.7-3.4)$ & $0.2(0.1-0.3)$ & $0.7(0.5-0.8)$ \\
\hline $15(9-21)$ & $22(20-24)$ & $4(2-6)$ & $41(32-51)$ & $0.4(0.3-0.6)$ & $3.8(3.4-4.1)$ & $0.2(0.1-0.3)$ & $0.8(0.6-1.0)$ \\
\hline $14(8-19)$ & $21(19-22)$ & $4(2-5)$ & $38(30-46)$ & $0.4(0.2-0.5)$ & $3.2(3.0-3.4)$ & $0.2(0.1-0.3)$ & $0.7(0.5-0.8)$ \\
\hline
\end{tabular}

\section{(Continued)}

${ }^{*} \mathrm{CVD}$ deaths include those from ischemic heart disease, ischemic stroke, and hypertensive heart disease.

†Diabetes mellitus deaths include deaths from the direct effects of SSBs on diabetes mellitus and the effects of SSBs on diabetes mellitus that are mediated through BMI.

‡Cancer deaths include those from breast cancer, uterine cancer, esophageal cancer, colon and rectum cancers, pancreatic cancers, kidney cancers, and gall bladder cancer.

$\S$ Total deaths include those from CVD, diabetes mellitus, and cancers as described above.

generations consuming higher intakes than older generations). If the latter is even partly contributing, then global mortality and morbidity attributable to SSBs may steeply rise as current generations age into higher risk of chronic diseases while continuing their higher intakes of SSBs.

This investigation had several strengths. We used global dietary data based on individual-level, largely nationally representative surveys, and assessed and adjusted for comparability, consistency, bias, and missing data across surveys. We evaluated and incorporated the best available evidence for effects of SSBs on BMI and diabetes mellitus and of BMI on adiposity-related diseases. We performed sensitivity analyses to assess the potential for overestimation of effects of individual diet components on chronic disease through comparison with dietary pattern studies based on both observational data and randomized trials. These sensitivity analyses indicated that the effects of dietary patterns on CHD predicted from the effects of individual dietary components on CHD were very similar to those observed both in prospective cohort studies evaluating associations of overall dietary patterns on CHD 
Proportion of combined mortality from diabetes, CVD, and cancers attributable to SSBs in 2010, by age and sex

A

B

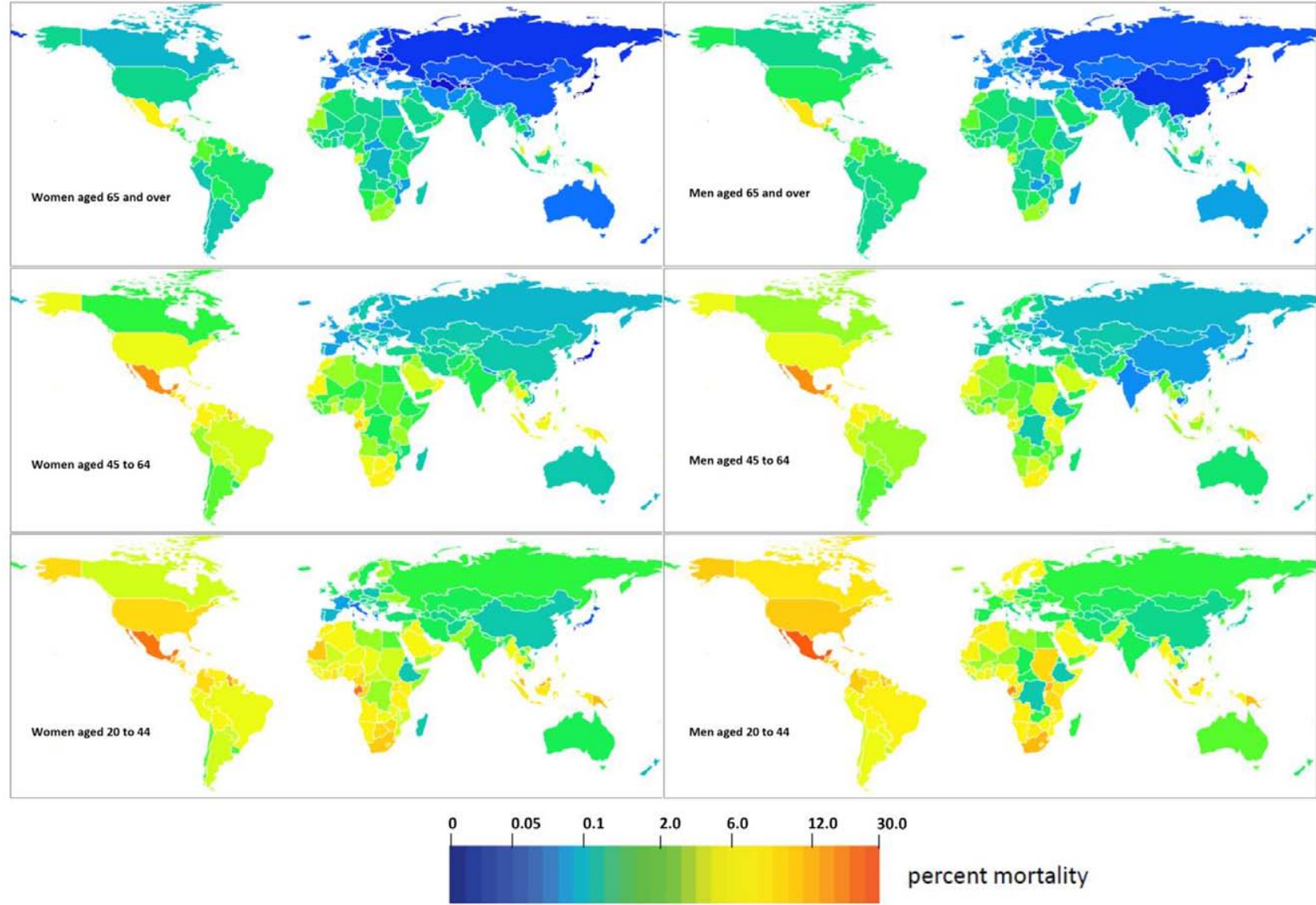

Figure 2. Proportion of combined mortality from diabetes mellitus, CVD, and cancers that is attributable to SSBs in 2010 in 3 age strata for women (A) and men (B). The color scale of each map indicates the proportional mortality for the given age-sex stratum in each country of the world, highlighting the inverse age gradient. *Proportional mortality was determined by summing SSB-attributable mortality across the outcomes of interest (diabetes mellitus, CVD, and cancers), and then dividing by the total number of deaths caused by these outcomes within the population of interest. CVD indicates cardiovascular disease; and SSB, sugar-sweetened beverages.

and randomized controlled studies of the impact of dietary patterns on CVD risk factors and CHD. Therefore, in combination, these sensitivity analyses confirm that the effects of single dietary factors on chronic diseases, such as those used in this study, are unlikely to overestimate the effects of diet on noncommunicable diseases attributable to residual confounding from other dietary components. Our analytic framework incorporated variation by age, sex, and country in SSB intakes, etiologic effects of SSBs on disease, and cause-specific mortality and morbidity, and also propagated uncertainty from each of these inputs into the final mortality estimates. Notably, our analyses did not simply involve comparisons of SSB intakes and disease rates, which would be limited by substantial confounding and ecological fallacy, but quantitatively assessed and incorporated age- and sex-specific data on SSB consumption and external evidence on the causal effects of SSBs on disease risk, and validated data on country, age, sex, and causespecific mortality.

Limitations should be considered. We did not identify national surveys on SSB consumption in some countries, especially those in South Asia and Sub-Saharan Africa, which limits the statistical certainty of estimates in these regions. Yet, no previous global data on individual-level SSB intakes have been compiled, ${ }^{19}$ and the dietary survey data we collected covered $62 \%$ of the world's population. We implemented hierarchical modeling methods with multiple informative time-varying covariates to account for missing data and to quantify its effects on uncertainty. Given our focus on chronic disease mortality, we did not collect SSB intakes in children and adolescents, among whom SSB consumption is likely higher than in young adults. Conversely, although current morbidity and future mortality attributable to SSBs may be appreciable even in youth, current mortality levels would be very low, producing little impact on our estimated burdens of deaths. Whereas effects of SSB intake on weight gain have been confirmed by randomized controlled trials and gene-diet interactions, ${ }^{2-4}$ the impact of BMI on chronic diseases was based on prospective observational studies, which might be overestimated because of residual confounding or underestimated owing to measurement error. Yet, these data represent the best available evidence for the effects of adiposity on chronic diseases, and the magnitude and causality of these 
20 countries with highest absolute mortality due to SSB consumption in 2010
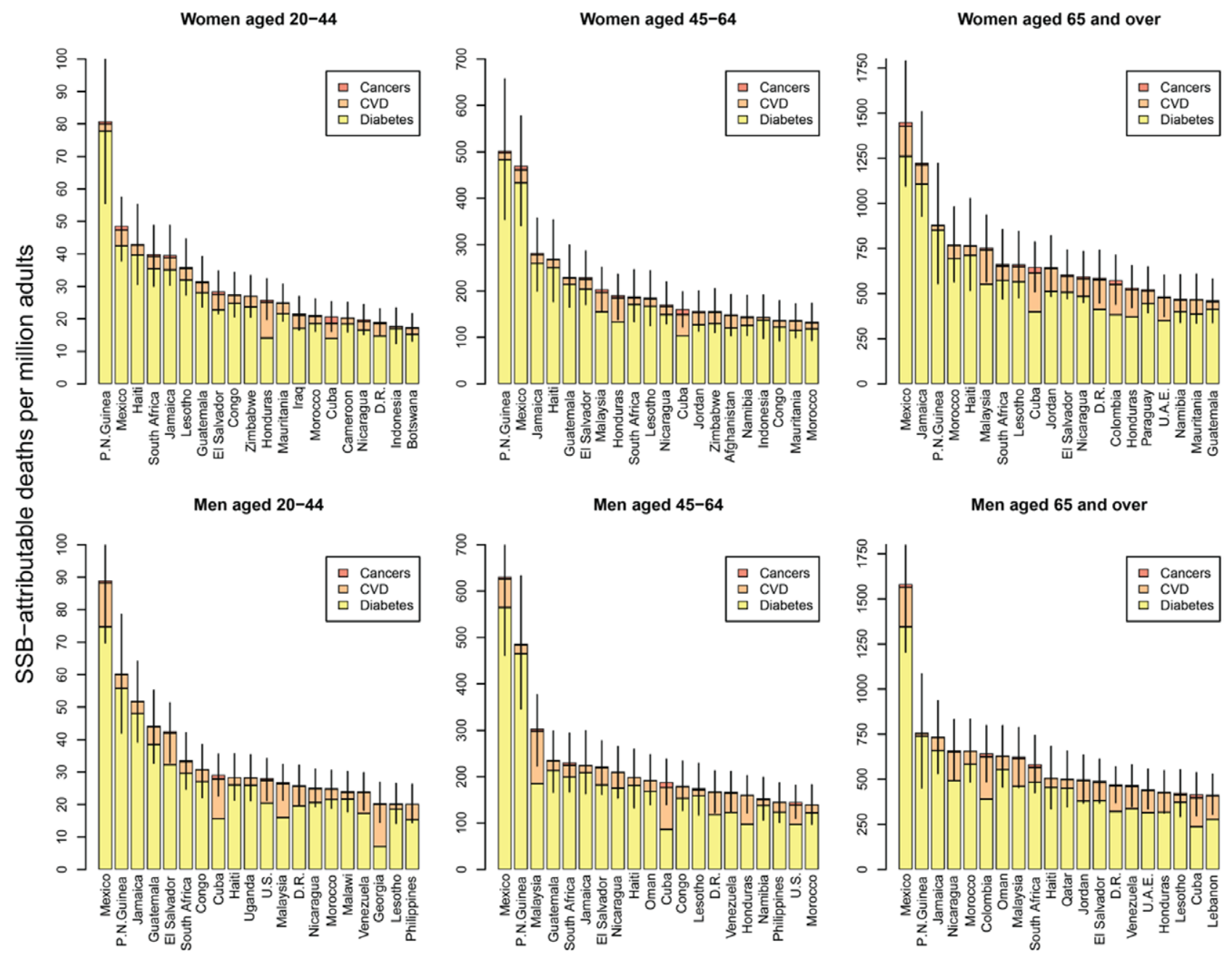

Figure 3. The 20 countries $^{*}$ with highest absolute SSB-attributable mortality in 2010 . Mortality is standardized per million adults.** The 20 countries selected in each age-sex group are those with highest SSB-attributable mortality AND with populations of at least 1 million. Note that $y$ axis scales differ in each panel. ${ }^{*} T$ The 47 smaller countries that were excluded because their populations were $<1$ million were Andorra, Antigua and Barbuda, Bahamas, Bahrain, Barbados, Belize, Bhutan, Brunei Darussalam, Cape Verde, China (Macao SAR), Comoros, Cyprus, Djibouti, Dominica, Equatorial Guinea, Fiji, French Polynesia, Gabon, Gambia, Grenada, Guadeloupe, GuineaBissau, Guyana, Iceland, Kiribati, Luxembourg, Maldives, Malta, Marshall Islands, Martinique, Mauritius, Micronesia, Montenegro, Netherlands Antilles, Réunion, Saint Lucia, Saint Vincent and the Grenadines, Samoa, São Tomé and Príncipe, Seychelles, Solomon Islands, Suriname, Swaziland, Timor-Leste, Tonga, Trinidad and Tobago, and Vanuatu. ${ }^{\star \star}$ Population-standardized absolute mortality was calculated by dividing attributable deaths by the adult population of the entity of interest (ie, country, region, or age-sex groups within a country or region) and then multiplying by 1 million. CVD indicates cardiovascular disease; D.R., Dominican Republic; P.N. Guinea, Papua New Guinea: SSB, sugar-sweetened beverages; and U.A.E., United Arab Emirates.

effects are supported by extensive experimental and shorterterm clinical interventions. ${ }^{24}$ In this analysis, we included only the BMI-mediated effects of SSB intake on CVD and cancers, because these effects have been well-established. ${ }^{24,30}$ However, SSB intake has been associated, independent of weight gain, with increases in inflammatory biomarkers and triglycerides, and the development of hypertension and hyperuricemia, as well, which may indicate direct effects on outcomes related to these pathways, such as CVD and cancers. ${ }^{33,47-50}$ Therefore, the death and DALY burdens presented here could be an underestimate of the true burden of SSB-related disease. Our analyses included diabetes mellitus mortality, but because diabetes mellitus is not often listed as the proximal cause of death, the total number of diabetes mellitus deaths reported here could be an underestimate. However, because many who have diabetes mellitus in life ultimately die of CVD causes, we include CVD mortality in our analyses, thereby mitigating possible underestimation of total SSB-related mortality.

Using a comparative risk assessment model, we found that in 2010, 184000 deaths and 8.5 million DALYs worldwide were attributable to consumption of sugary beverages, with three-quarters of these burdens occurring in low- and middleincome countries and the highest proportional burdens among adults 20 to 44 years of age. These results indicate the need for population-based efforts to reduce SSB consumption throughout the world through effective health policies and targeted interventions directed at stemming obesity-related disease. 
Table 4. Global and Regional DALYs Related to SSB Consumption in 2010

\begin{tabular}{|c|c|c|c|c|c|c|c|}
\hline & \multicolumn{3}{|c|}{ Population Characteristics } & \multicolumn{4}{|c|}{ Number of DALYs Attributable to SSBs ( $95 \%$ UI) } \\
\hline & Population & $\begin{array}{c}\text { Mean } \\
\text { SSB Intake } \\
\text { (Servings/d) }\end{array}$ & $\begin{array}{c}\text { Mean } \\
\text { BMI }\left(\mathrm{kg} / \mathrm{m}^{2}\right)\end{array}$ & CVD $\ddagger$ & Diabetes Mellitus§ & Cancers $\beta$ & Musculoskeletalø \\
\hline \multicolumn{8}{|l|}{ Globe } \\
\hline \multicolumn{8}{|l|}{ Women } \\
\hline Ages 20-44 & 987 & $0.8(0.3-1.3)$ & $24.7(23.1-26.3)$ & 294808 (53872-894390) & $372240(140880-769120)$ & $34343(6024-132271)$ & 33489 (5007-143503) \\
\hline Ages 45-64 & 654 & $0.4(0.2-0.6)$ & $27.2(25.2-29.1)$ & 519525 (77623-1416485) & 712098 (280473-1406840) & 87985 (15112-264554) & $132295(18392-411280)$ \\
\hline Ages $\geq 65$ & 293 & $0.3(0.1-0.5)$ & $25.8(22.8-28.7)$ & 742527 (121 255-2136253) & 595440 (239959-1174648) & $68755(11181-236558)$ & $88073(10524-327831)$ \\
\hline Women overall & 1930 & $0.5(0.2-0.8)$ & $25.7(23.5-27.9)$ & $1556860(252750-4447127)$ & 1679778 (661311-3350608) & $191083(32317-633384)$ & 253857 (33923-882614) \\
\hline \multicolumn{8}{|l|}{ Men } \\
\hline Ages 20-44 & 1020 & $0.9(0.4-1.4)$ & $24.3(22.9-25.8)$ & 619039 (118456-1602534) & $465992(179272-941049)$ & 24712 (4043-76731) & 21364 (3397-62462) \\
\hline Ages 45-64 & 645 & $0.4(0.2-0.7)$ & $25.8(24.1-27.5)$ & 1230704 (180660-2779689) & 843033 (335415-1635657) & 103247 (14853-250627) & 94963 (12361-267311) \\
\hline Ages $\geq 65$ & 231 & $0.3(0.1-0.5)$ & 24.7 (22.7-26.8) & 812458 (226030-2424935) & 517800 (209314-994 182) & 67653 (15239-304601) & 43914 (7232-176926) \\
\hline Men overall & 1890 & $0.6(0.2-0.9)$ & $24.8(23.1-26.6)$ & 2662201 (525146-6807159) & 1826824 (724002-3570888) & $195612(34136-631960)$ & 160241 (22989-506700) \\
\hline Both sexes overall & 3830 & $0.5(0.2-0.9)$ & $25.3(23.3-27.3)$ & 4219061 (777896-11254286) & 3506602 (1385313-6921496) & 386695 (66453-1265343) & 414097 (56912-1389314) \\
\hline \multicolumn{8}{|l|}{ Country income level } \\
\hline High income & 763 & $0.5(0.3-0.6)$ & $26.7(25.0-28.5)$ & 280243 (167 025-583031) & 881425 (394 880-1 562988) & 50087 (25564-133452) & 44740 (15407-155255) \\
\hline Upper middle income & 1530 & $0.7(0.3-1.1)$ & $26.3(24.4-28.2)$ & 619316 (203775-2447285) & 1376986 (568380-2649568) & 69435 (17044-365715) & 79980 (19886-345074) \\
\hline $\begin{array}{l}\text { Lower middle } \\
\text { income }\end{array}$ & 1210 & $0.6(0.2-1.0)$ & $25.2(23.2-27.2)$ & 2753266 (375163-6534363) & 1074097 (370326-2298304) & 211883 (21492-572887) & 204356 (18242-608452) \\
\hline Low income & 323 & $0.3(0.1-0.5)$ & $22.1(19.8-24.4)$ & 566236 (31933-1689606) & 174095 (51727-410636) & $55290(2353-193290)$ & 85021 (3377-280533) \\
\hline \multicolumn{8}{|l|}{ Australia and New Zealand } \\
\hline \multicolumn{8}{|l|}{ Women } \\
\hline Ages 20-44 & 3.6 & $0.4(0.2-0.6)$ & $26.0(25.2-26.9)$ & $173(106-259)$ & $554(206-1191)$ & $53(33-80)$ & $39(18-74)$ \\
\hline Ages 45-64 & 3.35 & $0.2(0.1-0.3)$ & $28.8(27.7-29.9)$ & 319 (219-457) & $1148(432-2503)$ & $180(122-256)$ & $182(83-344)$ \\
\hline Ages $\geq 65$ & 1.94 & $0.1(0.1-0.2)$ & $27.8(26.9-28.8)$ & $644(474-853)$ & $1396(544-2927)$ & $150(100-213)$ & $96(46-177)$ \\
\hline Women overall & 8.89 & $0.2(0.1-0.4)$ & $27.4(26.4-28.4)$ & $1136(800-1569)$ & 3097 (1183-6621) & 383 (254-548) & 317 (147-595) \\
\hline \multicolumn{8}{|l|}{ Men } \\
\hline Ages 20-44 & 3.57 & $0.4(0.2-0.6)$ & $26.9(26.2-27.6)$ & $410(267-601)$ & $713(257-1529)$ & $36(20-59)$ & $25(12-48)$ \\
\hline Ages 45-64 & 3.28 & $0.2(0.1-0.3)$ & $28.9(28.2-29.7)$ & $872(626-1176)$ & $1628(611-3414)$ & $194(123-281)$ & $117(55-224)$ \\
\hline Ages $\geq 65$ & 1.64 & $0.1(0.1-0.2)$ & $27.9(27.1-28.6)$ & $830(615-1091)$ & $1672(633-3439)$ & 177 (114-255) & $53(25-102)$ \\
\hline Men overall & 8.48 & $0.2(0.1-0.4)$ & $27.8(27.0-28.5)$ & 2112 (1509-2868) & 4012 (1501-8382) & 407 (256-595) & $196(92-374)$ \\
\hline Both sexes overall & 17.4 & $0.2(0.1-0.4)$ & $27.6(26.7-28.4)$ & 3248 (2308-4437) & 7109 (2683-15003) & $790(511-1144)$ & 513 (239-969) \\
\hline \multicolumn{8}{|l|}{ Canada and United States } \\
\hline \multicolumn{8}{|l|}{ Women } \\
\hline Ages 20-44 & 45 & $1.6(1.2-2.0)$ & $26.4(25.7-27.0)$ & 10059 (6559-13985) & 46638 (22 427-79711) & 1608 (1022-2448) & $1297(632-2466)$ \\
\hline Ages 45-64 & 46.1 & $0.8(0.6-1.0)$ & $29.2(28.4-30.1)$ & 21516 (15138-28549) & 117050 (55317-198511) & $5942(4009-8354)$ & 6632 (3309-12439) \\
\hline Ages $\geq 65$ & 25.6 & $0.6(0.4-0.7)$ & $28.0(27.2-28.8)$ & 24760 (18351-32226) & 114732 (53922-194331) & $4240(2850-6161)$ & $3283(1648-6002)$ \\
\hline Women overall & 117 & $1.0(0.8-1.2)$ & $27.7(26.9-28.5)$ & 56335 (40048-74759) & 278420 (131 666-472553) & 11790 (7881-16963) & 11211 (5589-20906) \\
\hline \multicolumn{8}{|l|}{ Men } \\
\hline Ages $20-44$ & 46.3 & $1.8(1.3-2.2)$ & $27.2(26.6-27.7)$ & 21980 (14596-31212) & 64555 (30299-108124) & $1283(733-2170)$ & $948(450-1731)$ \\
\hline Ages 45-64 & 44.2 & $0.9(0.7-1.1)$ & $29.1(28.5-29.8)$ & 50846 (36756-66 835) & 160750 (74 845-268450) & $6624(4074-10105)$ & $4690(2251-8367)$ \\
\hline Ages $\geq 65$ & 19.4 & $0.6(0.5-0.8)$ & $27.9(27.3-28.5)$ & 28821 (21554-36 844) & 111961 (50940-188457) & 4076 (2550-6098) & $1766(862-3175)$ \\
\hline Men overall & 110 & $1.1(0.8-1.4)$ & $27.9(27.3-28.5)$ & 101648 (72907-134 891) & 337267 (156 083-565031) & 11983 (7357-18373) & 7404 (3563-13273) \\
\hline Both sexes overall & 226 & $1.1(0.8-1.3)$ & $27.8(27.1-28.5)$ & 157983 (112955-209650) & 615686 (287 750-1 037 584) & 23773 (15238-35336) & 18616 (9151-34 179) \\
\hline \multicolumn{8}{|l|}{ East and Southeast Asia } \\
\hline \multicolumn{8}{|l|}{ Women } \\
\hline Ages 20-44 & 341 & $0.9(0.4-1.3)$ & $24.9(23.4-26.4)$ & 43502 (5336-261 258) & 66098 (24 272-144241) & $7540(706-50420)$ & 8651 (679-74 288) \\
\hline Ages 45-64 & 238 & $0.4(0.2-0.7)$ & $26.6(24.7-28.5)$ & 52701 (7184-220 062) & 135713 (50776-273544) & 11095 (1499-48 858) & 23984 (2383-100452) \\
\hline Ages $\geq 65$ & 101 & $0.3(0.1-0.5)$ & $24.5(21.6-27.5)$ & 241385 (11010-970152) & 110547 (38953-245 152) & 25084 (1042-129905) & 36381 (1480-187636) \\
\hline Women overall & 680 & $0.5(0.2-0.9)$ & $25.2(23.1-27.3)$ & 337588 (23529-1 451472) & 312357 (114002-662937) & 43719 (3248-229183) & 69015 (4542-362376) \\
\hline
\end{tabular}




\begin{tabular}{|c|c|c|c|c|c|c|c|c|c|c|}
\hline & \multicolumn{5}{|c|}{ DALYs per Million Adults Attributable to SSBs ( $95 \%$ UI) } & \multicolumn{5}{|c|}{ Percentage of DALYs Attributable to SSBs (95\% UI) } \\
\hline Total† & CVD‡ & Diabetes Mellitus§ & Cancers $\beta$ & Musculoskeletalף & Total† & CVD‡ & $\begin{array}{l}\text { Diabetes } \\
\text { Mellitus§ }\end{array}$ & Cancers $\beta$ & Musculoskeletalף & Total $†$ \\
\hline
\end{tabular}

\begin{tabular}{|c|c|c|c|c|}
\hline $734880(260247-1760532)$ & $230(42-698)$ & $290(110-600)$ & $27(5-103)$ & $26(4-112)$ \\
\hline 1451903 (472003-3185858) & 795 (119-2167) & 1089 (429-2152) & $135(23-405)$ & $202(28-629$ \\
\hline 1494795 (461518-3634885) & 2537 (414-7300) & $2035(820-4014)$ & $235(38-808)$ & $301(36-112$ \\
\hline 3681578 (1 193768-8581 275) & 699 (113-1996) & $754(297-1504)$ & $86(15-284)$ & $114(15-396$ \\
\hline 1131107 (387637-2420754) & $466(89-1207)$ & 351 (135-709) & $19(3-58)$ & $16(3-47)$ \\
\hline 2271946 (656182-4517436) & $1907(280-4308)$ & $1307(520-2535)$ & $160(23-388)$ & $147(19-414$ \\
\hline 1441825 (532366-3725193) & $3510(977-10477)$ & $2237(904-4295)$ & $292(66-1316)$ & $190(31-764$ \\
\hline $\begin{array}{c}4844878 \\
(1576185-10663382)\end{array}$ & $1208(238-3088)$ & $829(328-1620)$ & $89(15-287)$ & $73(10-230)$ \\
\hline $\begin{array}{c}8526456 \\
(2769953-19244657)\end{array}$ & 952 (175-2539) & 791 (313-1561) & $87(15-285)$ & $93(13-313)$ \\
\hline 1256495 (699247-2292130) & $336(200-699)$ & $1057(473-1874)$ & $60(31-160)$ & $54(18-186)$ \\
\hline 2145716 (948946-5564122) & 353 (116-1397) & $786(324-1512)$ & 40 (10-209) & $46(11-197)$ \\
\hline 4243602 (998925-9058923) & 1902 (259-4514) & $742(256-1588)$ & $146(15-396)$ & $141(13-42$ \\
\hline 880643 (122835-2329483) & $1420(80-4238)$ & $437(130-1030)$ & $139(6-485)$ & $213(8-704)$ \\
\hline $818(456-1479)$ & $39(24-58)$ & $124(46-267)$ & $12(7-18)$ & $9(4-17)$ \\
\hline $1829(1060-3196)$ & $95(65-136)$ & $343(129-747)$ & $54(36-76)$ & $54(25-103)$ \\
\hline 2286 (1383-3792) & 331 (244-439) & $718(280-1505)$ & 77 (51-109) & $50(24-91)$ \\
\hline 4933 (2898-8467) & $116(82-161)$ & $317(121-678)$ & $39(26-56)$ & $32(15-61)$ \\
\hline $1184(682-2025)$ & $92(60-134)$ & $159(57-342)$ & $8(4-13)$ & $6(3-11)$ \\
\hline 2811 (1711-4663) & $266(191-359)$ & 496 (186-1041) & $59(37-86)$ & $36(17-68)$ \\
\hline 2732 (1670-4525) & $507(376-667)$ & $1022(387-2103)$ & $108(70-156)$ & $33(15-63)$ \\
\hline 6727 (4063-11213) & $225(161-305)$ & $427(160-893)$ & $43(27-63)$ & $21(10-40)$ \\
\hline 11660 (6962-19680) & $170(121-232)$ & $371(140-783)$ & $41(27-60)$ & $27(12-51)$ \\
\hline
\end{tabular}

59603 (34939-93195) 151139 (88769-236152) 147015 (86073-227384) 357756 (209781-556731)

88767 (52997-134444) 222910 (135121-330714) $146625(85561-223353)$ 458302 (273679-688510) 816058 (483460-1 245241)

125791 (37694-501434) 223492 (73317-596369) 413397 (67327-1465240) 762680 (178337-2563042)
178 (116-248) $467(329-620)$ 966 (716-1257) $440(313-584)$

377 (250-535) 1151 (832-1513) 1489 (1114-1904) $834(598-1107)$ $632(452-839)$
$827(398-1413) \quad 29(18-43)$ 2541 (1201-4309) $129(87-181)$ $4475(2103-7579) \quad 165(111-240)$ $2173(1028-3689) \quad 92(62-132)$

$1107(520-1855) \quad 22(13-37)$ $3638(1694-6076) \quad 150(92-229)$ $5785(2632-9737) \quad 211(132-315)$ 2768 (1281-4638) $98(60-151)$ $2463(1151-4151) \quad 95(61-141)$
$23(11-44)$ $144(72-270)$ $128(64-234)$ $88(44-163)$

$16(8-30)$ $106(51-189)$ $91(45-164)$ $61(29-109)$ $74(37-137)$

\section{$573(203-1373)$} $2221(722-4874) \quad 1.2(0.4-3.1) \quad 4.7(1.8-9.1) \quad 0.2(0.1-0.6) \quad 0.4(0.1-1.4) \quad 0.8(0.3-1.7)$ $\begin{array}{llllll}5108(1577-12420) & 0.9(0.2-3.0) & 4.2(1.6-8.2) & 0.3(0.0-1.0) & 0.5(0.1-2.0) & 0.8(0.3-2.1)\end{array}$ $1652(536-3851) \quad 1.5(0.5-4.2) \quad 4.1(1.6-8.1) \quad 0.2(0.1-0.7) \quad 0.4(0.1-1.3) \quad 0.7(0.3-1.5)$

$852(292-1823) \quad 2.9(1.0-6.9) \quad 6.1(2.3-12.1) \quad 0.2(0.0-0.6) \quad 0.1(0.0-0.5) \quad 0.5(0.2-0.9)$ 3521 (1017-7002) $\quad 1.4(0.4-4.1) \quad 4.9(1.9-9.5) \quad 0.2(0.0-0.8) \quad 0.5(0.1-1.6) \quad 0.8(0.3-1.7)$ $6230(2300-16095) \quad 1.1(0.2-3.2) \quad 3.7(1.4-7.2) \quad 0.2(0.0-0.8) \quad 0.5(0.1-1.8) \quad 0.8(0.3-1.8)$ $2198(715-4837) \quad 1.8(0.6-4.7) \quad 4.9(1.9-9.6) \quad 0.2(0.0-0.7) \quad 0.4(0.1-1.3) \quad 0.7(0.3-1.5)$ $1924(625-4342) \quad 1.6(0.5-4.5) \quad 4.5(1.7-8.9) \quad 0.2(0.1-0.7) \quad 0.4(0.1-1.3) \quad 0.7(0.3-1.5)$

1507 (838-2748) $\quad 1.0(0.6-2.4) \quad 4.4(1.8-8.2) \quad 0.1(0.1-0.3) \quad 0.1(0.0-0.4) \quad 0.5(0.3-1.1)$ $1225(542-3175) \quad 1.5(0.7-3.4) \quad 6.1(2.5-11.7) \quad 0.2(0.1-0.5) \quad 0.3(0.1-0.9) \quad 0.8(0.4-1.6)$ $2931(690-6258) \quad 1.6(0.5-4.8) \quad 4.6(1.6-9.6) \quad 0.2(0.0-0.8) \quad 0.4(0.1-1.4) \quad 0.8(0.3-1.8)$ $2209(308-5843) \quad 2.6(0.3-8.4) \quad 2.1(0.7-4.6) \quad 0.4(0.0-1.5) \quad 0.7(0.0-2.9) \quad 0.5(0.1-1.5)$

$183(102-331) \quad 1.1(0.7-1.5) \quad 1.2(0.4-2.6) \quad 0.1(0.1-0.2) \quad 0.0(0.0-0.1) \quad 0.1(0.1-0.2)$ $546(316-954) \quad 0.4(0.3-0.6) \quad 1.9(0.7-3.7) \quad 0.1(0.1-0.1) \quad 0.1(0.0-0.2) \quad 0.2(0.1-0.3)$ $1176(711-1950) \quad 0.2(0.2-0.3) \quad 1.6(0.6-3.2) \quad 0.1(0.0-0.1) \quad 0.1(0.0-0.1) \quad 0.2(0.1-0.3)$ $505(297-868) \quad 0.6(0.4-0.8) \quad 1.6(0.6-3.2) \quad 0.1(0.1-0.1) \quad 0.1(0.0-0.1) \quad 0.2(0.1-0.3)$

$264(152-452) \quad 1.3(0.9-1.7) \quad 2.7(1.0-5.6) \quad 0.1(0.0-0.1) \quad 0.0(0.0-0.0) \quad 0.2(0.1-0.3)$ $857(522-1422) \quad 0.5(0.4-0.7) \quad 1.8(0.7-3.6) \quad 0.1(0.0-0.1) \quad 0.1(0.0-0.1) \quad 0.2(0.2-0.4)$ $1670(1021-2766) \quad 0.2(0.2-0.3) \quad 1.3(0.5-2.6) \quad 0.1(0.0-0.1) \quad 0.0(0.0-0.1) \quad 0.2(0.1-0.3)$ $\begin{array}{llllll}716(433-1194) & 0.7(0.5-0.9) & 1.9(0.7-3.9) & 0.1(0.0-0.1) & 0.0(0.0-0.1) & 0.2(0.1-0.3)\end{array}$ $609(364-1028) \quad 0.6(0.4-0.8) \quad 1.7(0.7-3.5) \quad 0.1(0.0-0.1) \quad 0.0(0.0-0.1) \quad 0.2(0.1-0.3)$

\begin{tabular}{|c|c|c|c|c|c|c|c|c|c|}
\hline $100(12-603)$ & $153(56-333)$ & $17(2-116)$ & $20(2-172)$ & $290(87-1158)$ & $2.5(0.6-7.2)$ & $4.4(1.6-8.8)$ & $0.2(0.0-0.7)$ & $0.2(0.0-0.7)$ & $0.6(0.2-1.5)$ \\
\hline $221(30-923)$ & 569 (213-1148) & $47(6-205)$ & $101(10-421)$ & 938 (308-2502) & $1.1(0.2-3.7)$ & $5.4(2.1-10.6)$ & $0.2(0.0-0.7)$ & $0.5(0.1-1.9)$ & $1.1(0.4-2.7)$ \\
\hline 2398 (109-9638) & 1098 (387-2435) & $249(10-1291)$ & 361 (15-1864) & $4107(669-14556)$ & $1.0(0.1-3.7)$ & $4.5(1.7-9.0)$ & $0.3(0.0-1.1)$ & $0.7(0.1-2.7)$ & $1.1(0.3-2.7)$ \\
\hline $437(30-1880)$ & 405 (148-859) & $57(4-297)$ & $89(6-469)$ & $988(231-3320)$ & $1.5(0.3-4.9)$ & $4.8(1.8-9.5)$ & $0.2(0.0-0.8)$ & $0.4(0.1-1.8)$ & $\begin{array}{l}1.0(0.3-2.3) \\
\text { (Continued) }\end{array}$ \\
\hline
\end{tabular}

1057 (619-1652) $\quad 2.5(1.8-3.4) \quad 6.5(2.9-11.5) \quad 0.2(0.1-0.4) \quad 0.1(0.0-0.1) \quad 0.6(0.3-1.0)$ $3281(1927-5126) \quad 1.1(0.8-1.3) \quad 9.0(4.2-15.0) \quad 0.2(0.1-0.2) \quad 0.2(0.1-0.4) \quad 0.9(0.5-1.4)$ $5734(3357-8868) \quad 0.5(0.4-0.6) \quad 7.4(3.4-12.2) \quad 0.1(0.1-0.2) \quad 0.1(0.1-0.3) \quad 0.8(0.4-1.3)$ $2793(1638-4346) \quad 1.4(1.0-1.8) \quad 7.6(3.5-12.9) \quad 0.2(0.1-0.3) \quad 0.1(0.1-0.3) \quad 0.7(0.4-1.2)$

$1523(909-2306) \quad 3.1(2.2-4.2) \quad 12.9(5.8-21.8) 0.2(0.1-0.4) \quad 0.1(0.0-0.1) \quad 0.8(0.4-1.3)$ $5045(3058-7485) \quad 1.3(1.0-1.7) \quad 9.1(4.0-15.0) \quad 0.2(0.1-0.3) \quad 0.2(0.1-0.4) \quad 1.1(0.6-1.7)$ $7576(4421-11540) \quad 0.6(0.4-0.7) \quad 6.2(2.8-10.5) \quad 0.1(0.1-0.1) \quad 0.1(0.1-0.3) \quad 0.8(0.5-1.3)$ $3762(2246-5651) \quad 1.7(1.2-2.2) \quad 9.4(4.2-15.8) \quad 0.2(0.1-0.3) \quad 0.1(0.1-0.3) \quad 0.9(0.5-1.4)$ $3265(1934-4982) \quad 1.5(1.1-2.0) \quad 8.5(3.9-14.3) \quad 0.2(0.1-0.3) \quad 0.1(0.1-0.3) \quad 0.8(0.5-1.3)$ 
Table 4. Continued

\begin{tabular}{|c|c|c|c|c|c|c|c|}
\hline & \multicolumn{3}{|c|}{ Population Characteristics } & \multicolumn{4}{|c|}{ Number of DALYs Attributable to SSBs (95\% UI) } \\
\hline & Population & $\begin{array}{c}\text { Mean } \\
\text { SSB Intake } \\
\text { (Servings/d) }\end{array}$ & $\begin{array}{c}\text { Mean } \\
\text { BMl }\left(\mathrm{kg} / \mathrm{m}^{2}\right)\end{array}$ & CVD $\ddagger$ & Diabetes Mellitus§ & Cancers $\beta$ & Musculoskeletalף \\
\hline \multicolumn{8}{|l|}{ Men } \\
\hline Ages 20-44 & 352 & $0.9(0.4-1.4)$ & $24.1(22.8-25.5)$ & 88199 (12895-393677) & 76211 (29010-154587) & 4449 (494-19905) & $3536(432-13127)$ \\
\hline Ages 45-64 & 243 & $0.5(0.2-0.7)$ & $25.2(23.7-26.7)$ & $153823(16420-557260)$ & 145043 (54636-293663) & 13398 (1697-40947) & $16893(1570-60715)$ \\
\hline Ages $\geq 65$ & 84.6 & $0.3(0.2-0.5)$ & $23.9(22.0-25.8)$ & 236311 (16201-1 229854) & 85066 (32 508-176977) & 27019 (1619-215283) & $16121(942-102011)$ \\
\hline Men overall & 680 & $0.6(0.3-0.9)$ & $24.3(22.7-25.9)$ & 478333 (45516-2 180791) & 306319 (116153-625 226) & $44866(3810-276135)$ & $36550(2944-175853)$ \\
\hline Both sexes overall & 1360 & $0.6(0.3-0.9)$ & $24.8(22.9-26.6)$ & 815921 (69045-3632262) & $618677(230155-1288163)$ & $88585(7058-505318)$ & $105565(7486-538229)$ \\
\hline \multicolumn{8}{|l|}{ East/Central Eurasia } \\
\hline \multicolumn{8}{|l|}{ Women } \\
\hline Ages 20-44 & 60.4 & $0.4(0.2-0.6)$ & $24.3(22.3-26.3)$ & $6687(2880-17236)$ & 17253 (5092-43821) & $751(302-2019)$ & $757(263-1784)$ \\
\hline Ages 45-64 & 55.5 & $0.2(0.1-0.3)$ & $28.0(25.6-30.4)$ & $16929(8580-29657)$ & $33426(10337-85735)$ & $2790(1355-5077)$ & $3815(1350-8390)$ \\
\hline Ages $\geq 65$ & 32.5 & $0.1(0.1-0.2)$ & $27.6(24.0-31.1)$ & 39042 (14017-166 425) & 29247 (9463-70097) & $2253(856-7102)$ & 2656 (679-11622) \\
\hline Women overall & 148 & $0.2(0.1-0.4)$ & $26.5(23.8-29.2)$ & 62658 (25 477-213318) & 79926 (24 893-199653) & $5793(2513-14197)$ & 7228 (2292-21797) \\
\hline \multicolumn{8}{|l|}{ Men } \\
\hline Ages 20-44 & 59.7 & $0.4(0.2-0.6)$ & $25.3(23.5-27.0)$ & $25573(12021-50703)$ & 20702 (6121-51122) & 566 (213-1371) & $490(169-1192)$ \\
\hline Ages 45-64 & 47.3 & $0.2(0.1-0.3)$ & $27.2(25.3-29.2)$ & 51698 (25795-102375) & 32181 (9826-78698) & $3025(1308-6353)$ & $2208(756-5400)$ \\
\hline Ages $\geq 65$ & 17.6 & $0.1(0.1-0.2)$ & $26.4(24.0-28.8)$ & 30889 (12279-110774) & 16525 (5222-38531) & 1701 (673-4952) & $847(232-3141)$ \\
\hline Men overall & 125 & $0.3(0.1-0.4)$ & $26.2(24.2-28.2)$ & 108161 (50095-263852) & 69409 (21 169-168352) & $5293(2194-12675)$ & 3545 (1158-9734) \\
\hline Both sexes overall & 273 & $0.2(0.1-0.4)$ & $26.3(24.0-28.7)$ & 170819 (75572-477 170) & 149335 (46 062-368 005) & 11086 (4708-26872) & $10773(3449-31530)$ \\
\hline \multicolumn{8}{|c|}{ Latin America and Caribbean } \\
\hline \multicolumn{8}{|l|}{ Women } \\
\hline Ages 20-44 & 88.1 & $1.7(0.7-2.7)$ & $26.4(24.5-28.3)$ & 20231 (11217-34984) & 98436 (41 292-182 108) & 2985 (1449-6155) & 3673 (1510-7479) \\
\hline Ages 45-64 & 54.2 & $0.8(0.3-1.4)$ & $28.9(26.7-31.1)$ & 30068 (17678-51607) & 206008 (87 896-382 255) & 6269 (3230-11 478) & 12640 (5174-26028) \\
\hline Ages $\geq 65$ & 22.7 & $0.6(0.3-1.0)$ & $26.8(23.8-29.9)$ & 29093 (15669-59 087) & 164436 (69 037-306 255) & 3771 (1731-8177) & 4931 (1875-11 862) \\
\hline Women overall & 165 & $1.1(0.4-1.7)$ & $27.2(24.8-29.6)$ & 79392 (44564-145678) & 468879 (198225-870618) & $13025(6411-25810)$ & 21244 (8559-45369) \\
\hline \multicolumn{8}{|l|}{ Men } \\
\hline Ages 20-44 & 85.6 & $1.8(0.7-2.9)$ & $25.2(23.6-26.8)$ & 35314 (20390-58766) & 121160 (51509-224711) & 1606 (755-3095) & 2294 (926-4807) \\
\hline Ages 45-64 & 50.3 & $0.9(0.3-1.5)$ & $26.6(24.8-28.4)$ & 53094 (32118-89724) & 233094 (98549-432220) & 4061 (2139-7159) & 7829 (3174-16611) \\
\hline Ages $\geq 65$ & 17.9 & $0.7(0.3-1.1)$ & $25.2(23.1-27.4)$ & 33828 (19127-69167) & 142331 (58743-264 426) & 2597 (1333-5295) & 2691 (1008-6728) \\
\hline Men overall & 154 & $1.2(0.4-1.9)$ & $25.6(23.7-27.4)$ & 122237 (71635-217657) & 496584 (208800-921 357) & 8264 (4227-15549) & 12814 (5108-28146) \\
\hline Both sexes overall & 319 & $1.1(0.4-1.8)$ & $26.4(24.2-28.5)$ & 201629 (116199-363336) & 965464 (407 026-1 791 975) & $21289(10638-41358)$ & 34058 (13667-73516) \\
\hline \multicolumn{8}{|c|}{ North Africa and Middle East } \\
\hline \multicolumn{8}{|l|}{ Women } \\
\hline Ages 20-44 & 66.1 & $0.6(0.2-1.1)$ & $27.0(25.7-28.3)$ & 12731 (6052-25713) & $37908(13277-83700)$ & $982(461-1989)$ & $1246(498-2593)$ \\
\hline Ages 45-64 & 32.8 & $0.3(0.1-0.6)$ & $30.5(28.8-32.1)$ & 12492 (6428-21695) & 50952 (18414-110019) & $1110(566-1990)$ & 3770 (1480-7759) \\
\hline Ages $\geq 65$ & 11.2 & $0.3(0.1-0.4)$ & $28.3(25.8-30.8)$ & 9885 (4252-26 282) & 34536 (12924-72621) & $416(182-1059)$ & $1249(407-3710)$ \\
\hline Women overall & 110 & $0.4(0.1-0.7)$ & $28.4(26.5-30.2)$ & 35108 (16732-73690) & 123397 (44615-266 339) & 2508 (1210-5039) & $6265(2385-14062)$ \\
\hline \multicolumn{8}{|l|}{ Men } \\
\hline Ages 20-44 & 71.6 & $0.7(0.2-1.1)$ & $26.1(24.9-27.2)$ & 22944 (11640-43242) & 49709 (17626-106 374) & 504 (214-1049) & 891 (349-1901) \\
\hline Ages 45-64 & 33.5 & $0.3(0.1-0.6)$ & $27.8(26.5-29.1)$ & $21313(11343-35722)$ & 53721 (19533-112453) & 594 (276-1075) & $2525(978-5327)$ \\
\hline Ages $\geq 65$ & 9.67 & $0.3(0.1-0.4)$ & $26.4(24.7-28.1)$ & 10576 (5113-28404) & $29478(10930-60885)$ & 297 (133-788) & $671(228-2155)$ \\
\hline Men overall & 115 & $0.4(0.2-0.7)$ & $26.6(25.2-28.0)$ & $54833(28096-107367)$ & 132907 (48089-279713) & 1395 (623-2912) & 4088 (1555-9383) \\
\hline Both sexes overall & 225 & $0.4(0.2-0.7)$ & $27.5(25.9-29.1)$ & 89942 (44 828-181 058) & 256304 (92704-546 052) & 3903 (1833-7951) & 10352 (3940-23445) \\
\hline \multicolumn{8}{|l|}{ South Asia } \\
\hline \multicolumn{8}{|l|}{ Women } \\
\hline Ages 20-44 & 224 & $0.3(0.1-0.5)$ & $21.6(20.1-23.2)$ & 161612 (13397-421 698) & 63895 (19728-142776) & $16834(1267-57540)$ & 13509 (702-40691) \\
\hline Ages 45-64 & 122 & $0.2(0.0-0.3)$ & $22.1(20.3-24.0)$ & 331469 (14304-891 167) & 106165 (34 086-226 102) & $50720(2242-156917)$ & $63622(2267-191320)$ \\
\hline Ages $\geq 65$ & 40.1 & $0.1(0.0-0.2)$ & $20.6(18.0-23.2)$ & 332328 (46536-653573) & 74360 (28880-152361) & 25555 (2584-58 894) & 29896 (3312-68673) \\
\hline Women overall & 385 & $0.2(0.1-0.3)$ & $21.4(19.3-23.4)$ & 825409 (74 237-1 966 438) & 244420 (82694-521 238) & 93109 (6093-273351) & 107028 (6282-300 684) \\
\hline \multicolumn{8}{|l|}{ Men } \\
\hline Ages 20-44 & 238 & $0.4(0.1-0.6)$ & $21.2(19.6-22.7)$ & 361696 (33 983-832 009) & 82398 (26816-183165) & 12771 (1034-35964) & $9673(552-26553)$ \\
\hline Ages 45-64 & 126 & $0.2(0.0-0.3)$ & $21.6(19.8-23.5)$ & 807618 (41 130-1640736) & $140143(48397-292875)$ & $63770(2954-145853)$ & 47102 (1996-118867) \\
\hline
\end{tabular}




\begin{tabular}{|c|c|c|c|c|c|c|c|c|c|}
\hline \multirow[b]{2}{*}{ Total† } & \multicolumn{5}{|c|}{ DALYs per Million Adults Attributable to SSBs ( $95 \%$ UI) } & \multicolumn{4}{|c|}{ Percentage of DALYs Attributable to SSBs (95\% UI) } \\
\hline & CVD $\ddagger$ & Diabetes Mellitus§ & Cancers $\beta$ & Musculoskeletalף & Total $\dagger$ & CVD $\ddagger$ & $\begin{array}{l}\text { Diabetes } \\
\text { Mellitus§ }\end{array}$ & Cancers $\beta$ Musculoskeletal & Total $\nmid$ \\
\hline
\end{tabular}

172396 (53231-528216) 329156 (89301-871090) 364516 (61 902-1706625) 866068 (204434-3105931) 1628748 (382771-5668974)
$196(29-875)$ $632(67-2290)$ 2795 (192-14544) $615(59-2803)$ $526(45-2343)$
$169(64-343)$ $596(225-1207)$ 1006 (384-2093) 394 (149-804) $399(148-831)$
$10(1-44)$

$55(7-168)$ $320(19-2546)$ $58(5-355)$ 57 (5-326)
$8(1-29)$ $69(6-250)$ $191(11-1206)$ $47(4-226)$ $68(5-347)$
$383(118-1173) \quad 2.4(0.6-6.7) \quad 5.8(2.0-11.8) \quad 0.1(0.0-0.4) \quad 0.1(0.0-0.5) \quad 0.6(0.2-1.4)$ $1353(367-3580) \quad 1.4(0.3-4.3) \quad 5.6(2.0-11.0) \quad 0.2(0.0-0.6) \quad 0.5(0.1-1.7) \quad 1.1(0.4-2.5)$ $4311(732-20182) \quad 1.2(0.1-3.6) \quad 4.1(1.5-8.2) \quad 0.2(0.0-0.8) \quad 0.6(0.0-2.1) \quad 1.0(0.3-2.3)$ $1113(263-3992) \quad 1.7(0.3-4.9) \quad 5.2(1.8-10.3) \quad 0.2(0.0-0.6) \quad 0.4(0.0-1.4) \quad 0.9(0.3-2.1)$ $1051(247-3657) \quad 1.6(0.3-4.9) \quad 5.0(1.8-9.9) \quad 0.2(0.0-0.7) \quad 0.4(0.0-1.6) \quad 0.9(0.3-2.2)$
25448 (11085-57788)

56960 (28529-112668)

73197 (32657-237016)

155605 (72271-407 471)

47331 (24300-87712)

$89113(48274-164867)$

49962 (23995-144 178)

186407 (96568-396757)

342012 (168839-804228)

\section{$86(37-222)$}

$305(155-535)$

1201 (431-5119)

379 (154-1289)

331 (156-656) 1093 (545-2164) 1755 (698-6295) 761 (352-1856) 555 (246-1551)
$223(66-566)$ 603 (186-1546) 900 (291-2156) 483 (150-1207)

268 (79-662) 680 (208-1663) 939 (297-2189) 488 (149-1184) 485 (150-1196)
10 (4-26) 50 (24-92) 69 (26-218) 35 (15-86)

7 (3-18) 64 (28-134) 97 (38-281) 37 (15-89) 36 (15-87)
10 (3-23) 69 (24-151) 82 (21-358) 44 (14-132)

6 (2-15) 47 (16-114) 48 (13-178) $25(8-68)$ 35 (11-103)
328 (143-746) 2252 (1005-7291) 941 (437-2463)

$1.1(0.4-5.0) \quad 1.9(0.6-4.3) \quad 0.1(0.0-0.4) \quad 0.1(0.0-0.1) \quad 0.2(0.1-0.5)$ $0.3(0.2-0.6) \quad 2.5(0.9-5.1) \quad 0.1(0.0-0.1) \quad 0.1(0.0-0.3) \quad 0.3(0.1-0.5)$ $0.3(0.1-1.6) \quad 2.3(0.9-4.6) \quad 0.1(0.0-0.7) \quad 0.2(0.0-1.1) \quad 0.3(0.1-1.2)$ $0.6(0.2-2.4) \quad 2.2(0.8-4.7) \quad 0.1(0.0-0.4) \quad 0.1(0.0-0.5) \quad 0.3(0.1-0.8)$ 1027 (514-2032)
125324 (65460-212885) 254985 (132029-440829) 202232 (100088-362843) 582542 (297578-1016557)

$160374(86326-268070)$ 298078 (157064-507815) 181446 (91 832-323350) 639899 (335222-1099235) 1222440 (632800-2115792)

52867 (25377-102851) 46086 (21 681-96060) $167278(80272-328058)$

74048 (36978-137613) 78153 (39911-140341) 41022 (20093-84738) 193223 (96982-362692) $360500(177254-690750)$ 68325 (33214-129147)
$177(98-307)$ 554 (326-952) 1281 (690-2602) 416 (233-763)

$316(183-527)$ 1056 (639-1784) 1889 (1068-3863) $680(398-1210)$ 544 (313-980)
$864(362-1598) \quad 26(13-54)$ 3799 (1621-7049) $116(60-212)$ $7243(3041-13489) \quad 166$ (76-360) $2456(1038-4560) \quad 68(34-135)$

1086 (462-2013) $\quad 14$ (7-28) 4634 (1959-8593) 81 (43-142) $7950(3281-14770) \quad 145(74-296)$ 2762 (1161-5124) 46 (24-86) 2604 (1098-4834) $57(29-112)$
32 (13-66) $233(95-480)$ 217 (83-522) $111(45-238)$

$21(8-43)$ $156(63-330)$ $150(56-376)$ $71(28-157)$ $92(37-198)$
$613(315-1136) \quad 1.0(0.5-2.6) \quad 4.3(1.5-8.8) \quad 0.1(0.0-0.2) \quad 0.0(0.0-0.1) \quad 0.2(0.1-0.5)$ $1884(1020-3485) \quad 0.4(0.2-1.2) \quad 2.7(1.0-5.6) \quad 0.1(0.0-0.2) \quad 0.1(0.0-0.4) \quad 0.3(0.1-0.6)$ $2839(1363-8193) \quad 0.3(0.1-1.3) \quad 1.8(0.7-3.7) \quad 0.1(0.0-0.4) \quad 0.1(0.0-0.6) \quad 0.2(0.1-1.0)$ $1311(679-2791) \quad 0.6(0.3-1.7) \quad 2.9(1.1-6.0) \quad 0.1(0.0-0.3) \quad 0.1(0.0-0.4) \quad 0.2(0.1-0.7)$ $1112(549-2615) \quad 0.6(0.2-2.0) \quad 2.6(0.9-5.4) \quad 0.1(0.0-0.3) \quad 0.1(0.0-0.4) \quad 0.2(0.1-0.7)$

$\begin{array}{cccc}145(69-292) & 431(151-951) & 11(5-23) & 14(6-29) \\ 381(196-661) & 1553(561-3353) & 34(17-61) & 115(45-236) \\ 886(381-2356) & 3096(1158-6509) & 37(16-95) & 112(36-333) \\ 266(127-558) & 935(338-2018) & 19(9-38) & 47(18-107) \\ & & & \\ 243(123-457) & 525(186-1125) & 5(2-11) & 9(4-20) \\ 635(338-1065) & 1602(582-3353) & 18(8-32) & 75(29-159) \\ 1094(529-2937) & 3048(1130-6296) & 31(14-82) & 69(24-223) \\ 398(204-779) & 964(349-2030) & 10(5-21) & 30(11-68) \\ 333(166-671) & 950(344-2024) & 14(7-29) & 38(15-87)\end{array}$

145 (69-292) $381(196-661)$ $886(381-2356)$
1099 (574-1868) $\quad 4.5(2.4-8.1) \quad 7.3(2.9-14.2) \quad 0.5(0.2-1.1) \quad 0.3(0.1-0.6) \quad 0.9(0.4-1.6)$ $4702(2435-8129) \quad 2.0(1.1-3.4) \quad 10.3(4.3-19.2) 0.4(0.2-0.7) \quad 0.7(0.3-1.6) \quad 1.8(0.9-3.2)$ 8907 (4408-15981) $\quad 1.1(0.5-2.7) \quad 9.5(3.9-17.7) \quad 0.3(0.1-0.9) \quad 0.6(0.2-1.9) \quad 1.8(0.9-3.5)$ 3051 (1559-5324) $\quad 2.5(1.3-4.7) \quad 9.0(3.7-17.0) \quad 0.4(0.2-0.9) \quad 0.6(0.2-1.4) \quad 1.5(0.7-2.8)$

1437 (773-2402) $\quad 5.2(2.7-9.7) \quad 12.4(5.0-23.2) \quad 0.3(0.1-0.7) \quad 0.2(0.1-0.6) \quad 0.8(0.4-1.5)$ $5926(3123-10096) \quad 2.2(1.2-4.0) \quad 10.6(4.5-19.8) 0.2(0.1-0.5) \quad 0.7(0.2-1.6) \quad 1.6(0.8-2.8)$ $10135(5129-18061) \quad 1.2(0.6-3.3) \quad 8.2(3.4-15.6) \quad 0.2(0.1-0.5) \quad 0.6(0.2-2.0) \quad 1.5(0.7-3.0)$ $3559(1864-6113) \quad 2.9(1.5-5.7) \quad 10.4(4.3-19.5) 0.2(0.1-0.6) \quad 0.5(0.2-1.4) \quad 1.3(0.7-2.4)$ $3297(1707-5707) \quad 2.7(1.4-5.2) \quad 9.7(4.0-18.3) \quad 0.3(0.1-0.7) \quad 0.5(0.2-1.4) \quad 1.4(0.7-2.6)$
601 (288-1169) $\quad 1.4(0.7-2.7) \quad 4.2(1.6-8.4) \quad 0.2(0.1-0.3) \quad 0.1(0.0-0.2) \quad 0.5(0.2-1.0)$ $2082(1012-3936) \quad 0.6(0.3-1.0) \quad 5.3(2.0-10.5) \quad 0.1(0.1-0.2) \quad 0.2(0.1-0.5) \quad 0.9(0.4-1.6)$ 4131 (1943-8610) $\quad 0.3(0.2-0.9) \quad 4.3(1.7-8.7) \quad 0.1(0.0-0.3) \quad 0.2(0.1-0.7) \quad 0.8(0.3-1.6)$ $1268(608-2486) \quad 0.8(0.4-1.6) \quad 4.6(1.7-9.2) \quad 0.1(0.1-0.3) \quad 0.2(0.1-0.5) \quad 0.7(0.3-1.4)$

$783(391-1455) \quad 1.5(0.8-2.8) \quad 7.3(2.8-14.6) \quad 0.1(0.0-0.2) \quad 0.1(0.0-0.2) \quad 0.6(0.3-1.2)$ $2330(1190-4184) \quad 0.7(0.4-1.1) \quad 5.0(1.9-10.0) \quad 0.1(0.0-0.1) \quad 0.2(0.1-0.4) \quad 0.8(0.4-1.5)$ $4242(2078-8762) \quad 0.4(0.2-1.0) \quad 3.8(1.5-7.6) \quad 0.0(0.0-0.1) \quad 0.2(0.0-0.6) \quad 0.7(0.3-1.5)$ 1402 (704-2632) $\quad 0.8(0.4-1.6) \quad 5.4(2.1-10.7) \quad 0.1(0.0-0.1) \quad 0.1(0.0-0.4) \quad 0.7(0.3-1.4)$ $1336(657-2561) \quad 0.8(0.4-1.6) \quad 5.0(1.9-10.0) \quad 0.1(0.0-0.2) \quad 0.2(0.1-0.4) \quad 0.7(0.3-1.4)$
$255850(53752-579714) \quad 543(45-1418)$ $551976(69830-1304908) \quad 2727(118-7333)$ 462139 (102995-849463) $8292(1161-16307)$ 1269965 (226577-2734084) $1798(162-4284)$

466539 (92219-972543) $1141(107-2624)$ 1058632 (123445-2015267) 6409 (326-13021)
$215(66-480) \quad 57(4-193) \quad 45(2-137)$ $874(280-1860) \quad 417(18-1291) \quad 523(19-1574)$ 1855 (721-3801) 638 (64-1469) 746 (83-1713) $532(180-1135) \quad 203(13-595) \quad 233(14-655)$

$260(85-578) \quad 40(3-113) \quad 31(2-84)$ $1112(384-2324) \quad 506(23-1157) \quad 374(16-943)$ 
Table 4. Continued

\begin{tabular}{|c|c|c|c|c|c|c|c|}
\hline & \multicolumn{3}{|c|}{ Population Characteristics } & \multicolumn{4}{|c|}{ Number of DALYs Attributable to SSBs (95\% UI) } \\
\hline & Population & $\begin{array}{c}\text { Mean } \\
\text { SSB Intake } \\
\text { (Servings/d) }\end{array}$ & $\begin{array}{c}\text { Mean } \\
\text { BMI }\left(\mathrm{kg} / \mathrm{m}^{2}\right)\end{array}$ & CVD $\ddagger$ & Diabetes Mellitus§ & Cancers $\beta$ & Musculoskeletalq \\
\hline Ages $\geq 65$ & 36.5 & $0.1(0.0-0.2)$ & $20.7(18.7-22.8)$ & 402157 (138747-752272) & 74420 (28 191-152815) & $23845(7007-48948)$ & 15579 (3361-36 232) \\
\hline Men overall & 401 & $0.2(0.1-0.4)$ & $21.1(19.3-22.9)$ & $1571471(213861-3225017)$ & 296960 (103403-628856) & 100386 (10995-230765) & 72354 (5909-181 652) \\
\hline Both sexes overall & 786 & $0.2(0.1-0.4)$ & $21.2(19.3-23.2)$ & $2396880(288098-5191455)$ & 541380 (186 097-1 150 094) & $193494(17088-504117)$ & $179382(12191-482335)$ \\
\hline \multicolumn{8}{|l|}{ Sub-Saharan Africa } \\
\hline \multicolumn{8}{|l|}{ Women } \\
\hline Ages 20-44 & 102 & $0.7(0.2-1.2)$ & $23.4(22.0-24.8)$ & $37029(6917-111725)$ & 30816 (10209-70855) & $2912(412-10073)$ & $3804(485-13033)$ \\
\hline Ages 45-64 & 46 & $0.3(0.1-0.6)$ & $24.7(22.9-26.5)$ & 49411 (5112-166467) & 42357 (15102-91 405) & $7557(614-28181)$ & 15404 (1348-60247) \\
\hline Ages $\geq 65$ & 15.1 & $0.2(0.1-0.4)$ & $23.2(20.0-26.4)$ & 52327 (3299-190299) & $31662(10880-68345)$ & $4481(259-18357)$ & $7791(365-33591)$ \\
\hline Women overall & 163 & $0.4(0.1-0.8)$ & $23.6(21.5-25.8)$ & 138767 (15328-468 492) & 104835 (36 190-230604) & 14950 (1285-56611) & 26999 (2198-106871) \\
\hline \multicolumn{8}{|l|}{ Men } \\
\hline Ages 20-44 & 103 & $0.8(0.2-1.3)$ & $22.1(20.4-23.7)$ & 57263 (9026-184 052) & 35051 (11 296-81 630) & $3024(329-12305)$ & $3166(357-12434)$ \\
\hline Ages 45-64 & 42.5 & $0.4(0.1-0.6)$ & $23.0(21.1-24.8)$ & 78552 (7711-267908) & 46511 (16286-98467) & $8853(711-34537)$ & 12168 (937-49004) \\
\hline Ages $\geq 65$ & 12.3 & $0.3(0.1-0.5)$ & $22.2(19.9-24.6)$ & 55345 (3270-177 770) & $24201(8211-51487)$ & $5327(287-19051)$ & $5388(223-21842)$ \\
\hline Men overall & 158 & $0.5(0.1-0.8)$ & $22.4(20.4-24.3)$ & 191160 (20007-629731) & 105762 (35794-231 584) & $17203(1327-65893)$ & $20722(1518-83280)$ \\
\hline Both sexes overall & 320 & $0.5(0.1-0.8)$ & $23.0(20.9-25.1)$ & 329927 (35 336-1098222) & 210597 (71 984-462 188) & $32153(2612-122505)$ & $47721(3715-190151)$ \\
\hline \multicolumn{8}{|l|}{ Western Europe } \\
\hline \multicolumn{8}{|l|}{ Women } \\
\hline Ages 20-44 & 57.2 & $0.6(0.4-0.7)$ & $24.2(22.6-25.7)$ & $2785(1408-7532)$ & $10643(4376-20716)$ & $678(372-1547)$ & $512(221-1096)$ \\
\hline Ages 45-64 & 55.9 & $0.2(0.2-0.3)$ & $27.6(25.7-29.5)$ & $4620(2981-6824)$ & $19279(8111-36767)$ & $2322(1474-3444)$ & $2246(998-4301)$ \\
\hline Ages $\geq 65$ & 42.9 & $0.2(0.1-0.2)$ & $27.7(25.4-30.1)$ & $13062(7646-37354)$ & $34525(15355-62560)$ & $2805(1576-6691)$ & 1791 (712-4558) \\
\hline Women overall & 156 & $0.4(0.3-0.5)$ & $26.4(24.4-28.3)$ & 20467 (12035-51710) & 64447 (27 843-120044) & 5806 (3422-11 682) & 4550 (1931-9955) \\
\hline \multicolumn{8}{|l|}{ Men } \\
\hline Ages 20-44 & 58.4 & $0.7(0.5-0.9)$ & $25.9(24.7-27.0)$ & 5658 (3637-8272) & $15493(6339-29806)$ & $474(251-814)$ & $340(150-668)$ \\
\hline Ages 45-64 & 54.8 & $0.3(0.2-0.3)$ & $28.2(26.9-29.6)$ & $12886(8760-17955)$ & 29963 (12733-55416) & $2728(1571-4317)$ & $1431(643-2797)$ \\
\hline Ages $\geq 65 \mathrm{r}$ & 31.9 & $0.2(0.1-0.3)$ & $27.5(25.9-29.1)$ & $13700(9123-18760)$ & 32147 (13937-57 165) & 2614 (1523-3930) & $797(350-1540)$ \\
\hline Men overall & 145 & $0.4(0.3-0.5)$ & $27.1(25.7-28.5)$ & 32245 (21 520-44 986) & 77603 (33010-142387) & $5817(3345-9061)$ & $2569(1143-5005)$ \\
\hline Both sexes overall & 301 & $0.4(0.3-0.5)$ & $26.7(25.1-28.4)$ & 52712 (33555-96696) & 142050 (60853-262 431) & $11622(6767-20743)$ & 7118 (3074-14960) \\
\hline
\end{tabular}

Countries are grouped into regions as follows: Australia/New Zealand - Australia, New Zealand; East/Central Eurasia - Albania, Armenia, Azerbaijan, Bulgaria, Bosnia and Herzegovina, Belarus, Czech Republic, Estonia, Georgia, Croatia, Hungary, Kazakhstan, Kyrgyzstan, Lithuania, Latvia, Moldova, Macedonia, Montenegro, Mongolia, Poland, Romania, Russian Federation, Serbia, Slovakia, Slovenia, Tajikistan, Turkmenistan, Ukraine, Uzbekistan; East/Southeast Asia - Brunei Darussalam, China, Fiji, Micronesia, Indonesia, Japan, Cambodia, Kiribati, Republic of Korea, Lao People's Democratic Republic, Sri Lanka, Maldives, Marshall Islands, Myanmar, Malaysia, Philippines, Papua New Guinea, Democratic People's Republic of Korea, Singapore, Solomon Islands, Thailand, Timor-Leste, Tonga, Taiwan, Viet Nam, Vanuatu, Samoa; Latin America/Caribbean - Argentina, Antigua and Barbuda, Bahamas, Belize, Bolivia, Brazil, Barbados, Chile, Colombia, Costa Rica, Cuba, Dominica, Dominican Republic, Ecuador, Grenada, Guatemala, Guyana, Honduras, Haiti, Jamaica, Saint Lucia, Mexico, Nicaragua, Panama, Peru, Paraguay, El Salvador, Suriname, Trinidad and Tobago, Uruguay, Saint Vincent and the Grenadines, Venezuela; North Africa/Middle East - United Arab Emirates, Bahrain, Algeria, Egypt, Iran, Iraq, Jordan, Kuwait, Lebanon, Libyan Arab Jamahiriya, Morocco, Oman, Occupied Palestinian Territory, Qatar, Saudi Arabia, Syrian Arab Republic, Tunisia, Turkey, Yemen; South Asia - Afghanistan, Bangladesh, Bhutan, India, Nepal, Pakistan; SubSaharan Africa - Angola, Burundi, Benin, Burkina Faso, Botswana, Central African Republic, Côte d'Ivoire, Cameroon, Democratic Republic of the Congo, Congo, Comoros, Cape Verde, Djibouti, Eritrea, Ethiopia, Gabon, Ghana, Guinea, Gambia, Guinea-Bissau, Equatorial Guinea, Kenya, Liberia, Lesotho, Madagascar, Mali, Mozambique, Mauritania, Mauritius, Malawi, Namibia, Niger, Nigeria, Rwanda, Sudan, Senegal, Sierra Leone, Somalia, São Tomé and Príncipe, Swaziland, Seychelles, Chad, Togo, United Republic of Tanzania, Uganda, South Africa, Zambia, Zimbabwe; Western Europe - Andorra, Austria, Belgium, Switzerland, Cyprus, Germany, Denmark, Spain, Finland, France, United Kingdom, Greece, Ireland, Iceland, Israel, Italy, Luxembourg, Malta, Netherlands, Norway, Portugal, Sweden. BMl indicates body mass index; CVD, cardiovascular disease; DALY, disability-adjusted life year; SSB, sugar-sweetened beverages; and UI, uncertainty interval.

\section{Acknowledgments}

The Global Burden of Diseases Nutrition and Chronic Disease Expert Group (NutriCoDE) authorship group includes the following: Core Group: Dariush Mozaffarian, MD, DrPh, Renata Micha, PhD, and Peilin Shi, PhD, all at Tufts University Friedman School of Nutrition Science \& Policy, Boston, MA, USA; Majid Ezzati, PhD, Imperial College London, London, UK; Saman Fahimi, MD, MPH, University of Cambridge, Cambridge, UK; Shahab Khatibzadeh, MD, MPH, Harvard School of Public Health, Boston, MA, USA; John Powles, MB, BS, MA, FFPH, University of Cambridge, Cambridge, UK. Other Members: Ibrahim Elmadfa, PhD, Institute of Nutritional Sciences, University of Vienna, Vienna, Austria; Mayuree Rao, BA,
The Warren Alpert Medical School of Brown University, Providence, RI, USA; Pattra Wirojratana, Harvard School of Public Health, Boston, MA, USA; Gitanjali Singh, PhD, Tufts University Friedman School of Nutrition Science \& Policy, Boston, MA USA.

Dietary Exposure Imputation: Stephen S. Lim, PhD, Institute for Health Metrics and Evaluation, University of Washington, Seattle, Washington, USA; Kathryn G. Andrews, MPH, African Leaders Malaria Alliance, Dar es Salaam, Tanzania; Rebecca E Engell, BA, Institute for Health Metrics and Evaluation, University of Washington, Seattle, Washington, USA.

Dietary Exposures - Corresponding Members (alphabetic order): Pamela A. Abbott, PhD, University of Aberdeen, United Kingdom; 


\begin{tabular}{|c|c|c|c|c|c|c|c|c|c|c|}
\hline \multirow[b]{2}{*}{ Total† } & \multicolumn{5}{|c|}{ DALYs per Million Adults Attributable to SSBs (95\% UI) } & \multicolumn{5}{|c|}{ Percentage of DALYs Attributable to SSBs (95\% UI) } \\
\hline & CVD $\ddagger$ & Diabetes Mellitus§ & Cancers $\beta$ & Musculoskeletalף & Total† & CVD $\ddagger$ & $\begin{array}{l}\text { Diabetes } \\
\text { Mellitus§ }\end{array}$ & Cancers $\beta$ & Musculoskeletalף & Total† \\
\hline 516000 (201633-914121) & $\begin{array}{c}11026 \\
(3804-20624)\end{array}$ & $2040(773-4190)$ & $\begin{array}{c}654 \\
(192-1342)\end{array}$ & 427 (92-993) & $14147(5528-25062)$ & $2.6(0.3-6.3)$ & $2.0(0.7-4.2)$ & $0.5(0.1-1.5)$ & $0.8(0.1-2.7)$ & $0.9(0.2-2.1)$ \\
\hline 2041171 (417298-3901 931) & $3277(446-6725)$ & $619(216-1311)$ & $209(23-481)$ & $151(12-379)$ & $4257(870-8137)$ & $3.7(0.3-9.7)$ & $2.5(0.9-5.3)$ & $0.5(0.0-1.5)$ & $0.6(0.0-2.1)$ & $0.8(0.1-1.9)$ \\
\hline 3311136 (643875-6636016) & $2554(307-5531)$ & 577 (198-1225) & $206(18-537)$ & $191(13-514)$ & $3528(686-7070)$ & $3.5(0.3-9.5)$ & $2.3(0.8-4.9)$ & $0.6(0.0-1.8)$ & $0.7(0.0-2.4)$ & $0.8(0.1-2.0)$ \\
\hline 74561 (23857-182806) & $261(49-789)$ & $218(72-500)$ & $21(3-71)$ & $27(3-92)$ & $526(168-1291)$ & $2.5(0.6-8.2)$ & $1.9(0.6-4.2)$ & $0.2(0.0-0.7)$ & $0.2(0.0-0.7)$ & $0.2(0.1-0.5)$ \\
\hline 114729 (28738-315748) & $1075(111-3622)$ & $922(329-1989)$ & $164(13-613)$ & $335(29-1311)$ & $2496(625-6871)$ & $1.4(0.2-5.4)$ & $2.7(0.9-5.7)$ & $0.2(0.0-0.9)$ & $0.6(0.1-2.5)$ & $0.5(0.1-1.4)$ \\
\hline 96260 (18857-288885) & $3466(219-12603)$ & $2097(721-4526)$ & $297(17-1216)$ & $516(24-2225)$ & $6375(1249-19133)$ & $1.4(0.1-5.4)$ & $2.6(0.9-5.6)$ & $0.4(0.0-1.5)$ & $0.8(0.0-3.6)$ & $0.7(0.1-2.1)$ \\
\hline $285550(71452-787440)$ & $685(76-2312)$ & $517(179-1138)$ & $74(6-279)$ & $133(11-527)$ & 1409 (353-3885) & $1.8(0.3-6.3)$ & $2.4(0.8-5.2)$ & $0.3(0.0-1.1)$ & $0.5(0.0-2.3)$ & $0.5(0.1-1.3)$ \\
\hline 98504 (28433-253567) & $400(63-1286)$ & $245(79-570)$ & $21(2-86)$ & $22(2-87)$ & $688(199-1772)$ & $3.7(0.6-11.1)$ & $3.3(1.1-7.2)$ & $0.3(0.0-1.2)$ & $0.2(0.0-0.9)$ & $0.3(0.1-0.7)$ \\
\hline 146083 (32801-409279) & $1849(182-6307)$ & 1095 (383-2318) & $208(17-813)$ & $286(22-1154)$ & 3439 (772-9635) & $2.1(0.3-8.1)$ & $3.1(1.1-6.5)$ & $0.4(0.0-1.9)$ & $0.8(0.1-3.4)$ & $0.6(0.1-1.7)$ \\
\hline 90261 (15464-248934) & 4495 (266-14438) & $1966(667-4182)$ & $433(23-1547)$ & $438(18-1774)$ & 7331 (1256-20218) & $1.8(0.1-5.9)$ & $2.3(0.8-4.9)$ & $0.5(0.0-1.7)$ & $0.9(0.0-3.6)$ & $0.7(0.1-2.0)$ \\
\hline 334848 (76698-911779) & $966(101-3182)$ & $534(181-1170)$ & $87(7-333)$ & $105(8-421)$ & $1692(388-4607)$ & $2.6(0.3-8.4)$ & $2.9(1.0-6.2)$ & $0.4(0.0-1.6)$ & $0.6(0.0-2.6)$ & $0.5(0.1-1.4)$ \\
\hline 620398 (148150-1699219) & $824(88-2742)$ & $526(180-1154)$ & $80(7-306)$ & $119(9-475)$ & 1549 (370-4242) & $2.2(0.3-7.3)$ & $2.6(0.9-5.7)$ & $0.3(0.0-1.3)$ & $0.6(0.0-2.5)$ & $0.5(0.1-1.4)$ \\
\hline 14618 (7626-28381) & $40(20-108)$ & $153(63-298)$ & $10(5-22)$ & $7(3-16)$ & $210(110-408)$ & $1.7(0.8-5.0)$ & $2.1(0.8-4.2)$ & $0.1(0.1-0.4)$ & $0.0(0.0-0.1)$ & $0.2(0.1-0.4)$ \\
\hline 28468 (16517-46842) & $83(53-122)$ & $345(145-658)$ & $42(26-62)$ & $40(18-77)$ & 509 (295-838) & $0.5(0.3-0.7)$ & $2.8(1.2-5.2)$ & $0.1(0.1-0.1)$ & $0.1(0.0-0.1)$ & $0.2(0.1-0.4)$ \\
\hline 52183 (30457-104202) & $305(178-872)$ & $806(358-1460)$ & $65(37-156)$ & $42(17-106)$ & $1218(711-2431)$ & $0.3(0.1-0.7)$ & $2.8(1.2-4.9)$ & $0.1(0.0-0.2)$ & $0.1(0.0-0.2)$ & $0.3(0.2-0.6)$ \\
\hline 95269 (54601-179425) & $122(72-307)$ & $383(165-713)$ & $34(20-69)$ & $27(11-59)$ & $566(324-1066)$ & $0.8(0.4-2.1)$ & $2.6(1.1-4.8)$ & $0.1(0.1-0.3)$ & $0.1(0.0-0.1)$ & $0.2(0.1-0.5)$ \\
\hline 21966 (12471-36564) & $80(51-116)$ & $218(89-419)$ & $7(4-11)$ & $5(2-9)$ & $309(175-514)$ & $1.4(0.9-2.1)$ & $5.4(2.1-10.1)$ & $0.1(0.0-0.2)$ & $0.0(0.0-0.0)$ & $0.3(0.1-0.5)$ \\
\hline 47008 (28553-73400) & $235(160-328)$ & 547 (232-1011) & $50(29-79)$ & $26(12-51)$ & $858(521-1340)$ & $0.5(0.3-0.7)$ & $3.0(1.2-5.5)$ & $0.1(0.0-0.1)$ & $0.1(0.0-0.1)$ & $0.3(0.2-0.6)$ \\
\hline 49259 (30216-75369) & $429(286-587)$ & $1007(436-1790)$ & $82(48-123)$ & $25(11-48)$ & $1543(946-2360)$ & $0.2(0.2-0.3)$ & $2.1(0.9-3.8)$ & $0.0(0.0-0.1)$ & $0.0(0.0-0.1)$ & $0.3(0.2-0.4)$ \\
\hline 118233 (71241-185333) & 204 (136-285) & 491 (209-902) & $37(21-57)$ & $16(7-32)$ & $749(451-1174)$ & $0.7(0.5-1.1)$ & $3.5(1.4-6.4)$ & $0.1(0.0-0.1)$ & $0.0(0.0-0.1)$ & $0.3(0.2-0.5)$ \\
\hline 213503 (125841-364758) & $162(103-296)$ & $435(187-805)$ & $36(21-64)$ & $22(9-46)$ & $655(386-1118)$ & $0.8(0.4-1.6)$ & $3.0(1.2-5.6)$ & $0.1(0.0-0.2)$ & $0.0(0.0-0.1)$ & $0.3(0.1-0.5)$ \\
\hline
\end{tabular}

\section{(Continued)}

${ }^{*}$ CVD DALYs include those from ischemic heart disease, ischemic stroke, and hypertensive heart disease.

†Diabetes mellitus DALYs include those from the direct effects of SSBs on diabetes mellitus and the effects of SSBs on diabetes mellitus that are mediated through BMI. $\ddagger$ Cancer DALYs include those from breast cancer, uterine cancer, esophageal cancer, colon and rectum cancers, pancreatic cancers, kidney cancers, and gall bladder cancer. $\S$ Musculoskeletal DALYS include those from lower back pain and osteoarthritis.

\|Total DALYs include those from CVD, diabetes mellitus, and cancers as described above.

Morteza Abdollahi, MD, MPH, National Nutrition and Food Technology Research Institute Iran; Enrique Abeyá Gilardon, MD, MPH, Ministerio de Salud, Argentina; Habibul Ahsan, MD, University of Chicago, USA; Mohannad Abed Alfattah Al Nsour, MD, Eastern Mediterranean Public Health Network (EMPHNET), Jordan; Suad N. Al-Hooti, MSc, Kuwait Institute for Scientific Research, Kuwait; Carukshi Arambepola, MD, Faculty of Medicine, University of Colombo, Sri Lanka; Hubert Barennes, PhD, Institut Francophone pour la Médecine Tropicale, Lao PDR; Simon Barquera, PhD, Instituto Nacional de Salud Publica (INSP), Mexico; Ana Baylin, $\mathrm{MD}$, DrPH, University of Michigan, USA; Wulf Becker, $\mathrm{PhD}$, National Food Agency, Sweden; Peter Bjerregaard, MD, DrMedSci,
National Institute of Public Health, University of Southern Denmark, Denmark; Lesley T. Bourne, PhD, Environment and Health Research Unit, Medical Research Council, South Africa; Neville Calleja, MD, MFPH, Department of Health Information \& Research, Malta; Mario V. Capanzana, PhD, Food and Nutrition Research Institute, Philippines; Katia Castetbon, $\mathrm{PhD}$, Institut de Veille Sanitaire, France; Hsing-Yi Chang, DrPH, National Health Research Institutes, Taiwan; Yu Chen, PhD, New York University School of Medicine, United States; Melanie J. Cowan, MPH, WHO, Switzerland; Stefaan De Henauw, MD, PhD, Ghent University, Department of Public Health, Belgium; Eric L. Ding, ScD, Harvard Medical School and Harvard School of Public Health, USA; Charmaine A. Duante, MSc, 
Food and Nutrition Research Institute, Department of Science and Technology, Philippines; Pablo Duran, PhD, Dirección Nacional de Maternidad e Infancia, Ministerio de Salud de la Nación, Argentina; Ibrahim Elmadfa, Professor, Institute of Nutritional Sciences, University of Vienna, Austria; Heléne Enghardt Barbieri, National Food Agency, Uppsala, Sweden; Farshad Farzadfar, MD, ScD, Tehran University of Medical Sciences, Iran; Dulitha N. Fernando, $\mathrm{PhD}$, Faculty of Medicine, University of Colombo, Sri Lanka; Aida Filipovic Hadziomeragic, MD, MSc, Institute of Public Health of Federation of Bosnia and Herzegovina, Bosnia and Herzegovina; Regina M. Fisberg, PhD, Faculty of Public Health, University of São Paulo, Brazil; Simon Forsyth, BA (UQ), School of Population Health, University of Queensland, Australia; Didier Garriguet, MSc, Statistics Canada, Canada; Jean-Michel Gaspoz, MD, MPH, Geneva University Hospitals and Faculty of Medicine of Geneva, Switzerland; Dorothy Gauci, Post Graduate Diploma (Epidemiology), Department of Health Information and Research, Malta; Brahmam N.V. Ginnela, $\mathrm{MB}$, BS, DPH, National Institute of Nutrition, Indian Council of Medical Research, India; Idris Guessous, MD, Geneva University Hospitals, Switzerland; Martin C. Gulliford, FFPH, King's College London, UK; Wilbur Hadden; Christian Haerpfer, PhD, University of Aberdeen, United Kingdom; Daniel J. Hoffman, PhD, Rutgers, the State University of New Jersey, USA; Anahita Houshiar-Rad MSc, National Nutrition and Food Technology Research Institute Shahid Beheshti University of Medical Sciences, Iran; Inge Huybrechts, PhD, Ghent University, Department of Public Health, Belgium; Nahla C. Hwalla, PhD, American University of Beirut, Lebanon; Hajah Masni Ibrahim, SM, Ministry of Health, Brunei; Manami Inoue, MD, PhD, Epidemiology and Prevention Division, Research Center for Cancer Prevention and Screening, National Cancer Center, Japan; Maria D. Jackson, PhD, University of the West Indies, Jamaica; Lars Johansson, $\mathrm{PhD}$, Norwegian Directorate of Health, Norway; Lital Keinan-Boker, $\mathrm{MD}, \mathrm{PhD}$, Ministry of Health, Israel; Cho-il Kim, PhD, Korea Health Industry Development Institute, Republic of Korea; Eda Koksal, $\mathrm{PhD}$, Gazi University, Turkey; Hae-Jeung Lee; Yanping Li, PhD, Harvard School of Public Health, USA; Nur Indrawaty Lipoeto, $\mathrm{PhD}$, Andalas University, Indonesia; Guansheng $\mathrm{Ma}, \mathrm{PhD}$, National Institute for Nutrition and Food Safety, Chinese Center for Disease Control and Prevention, China; Guadalupe L. Mangialavori, MS RD, Ministerio de Salud de la Nación (National Health Ministry), Argentina; Yasuhiro Matsumura, PhD, Bunkyo University, Japan; Stephen T. McGarvey, PhD, Brown University, USA; Mei Fen Chan; Gert B.M. Mensink, PhD, Robert Koch Institute, Germany; Rafael A. Monge-Rojas, $\mathrm{PhD}$, Costa Rican Institute for Research and Education and Nutrition and Health (INCIENSA), Costa Rica; Abdulrahman O. Musaiger, PhD, Arab Center for Nutrition, Bahrain; Nagalla Balakrishna, PhD, National Institute of Nutrition, Hyderabad, India; Androniki Naska, PhD, Dept. of Hygiene, Epidemiology and Medical Statistics, University of Athens Medical School, Greece; Marga C. Ocke, $\mathrm{PhD}$, National Institute for Public Health and the Environment, Netherlands; Maciej Oltarzewski, MSc, National Food and Nutrition Institute, Poland; Philippos Orfanos, MSc, Dept. of Hygiene, Epidemiology and Medical Statistics, University of Athens Medical School, Greece; Marja-Leena Ovaskainen, $\mathrm{PhD}$, National Institute for Health and Welfare, Finland; Wen-Harn Pan, PhD, Division of Preventive Medicine and Health Services Research, Institute of Population Health Sciences, National Health Research Institutes, Taiwan; Demosthenes B. Panagiotakos, PhD, Harokopio University, Greece; Gulden A. Pekcan, PhD, Hacettepe University Department of Nutrition and Dietetics, Turkey; Stefka Petrova, MD, PhD, National Center of Public Health and Analyses, Bulgaria; Noppawan Piaseu, PhD, Mahidol University, Thailand; Christos Pitsavos, MD, Athens University Medical School, Greece; Luz Gladys Posada, RD, MS, Universidad de Antioquia, Colombia; Leanne M. Riley, MSc, WHO, Switzerland; Luz Maria Sánchez-Romero, MD MSc, National Institute of Public Health, Mexico; Rusidah BT. Selamat, MSc, Nutrition Division, Ministry of Health, Malaysia; Sangita Sharma; Abla Mehio Sibai, PhD, American University of Beirut Faculty of Health Sciences, Lebanon; Rosely Sichieri, MD, PhD, State University of Rio de Janeiro, Brazil; Chansimaly Simmala, MD, Institut of Tropical Medecin, Laos; Laufey Steingrimsdottir, PhD, Professor,
Iceland; Gillian Swan; Elżbieta Halina. Sygnowska, MSc PhD, National Institute of Cardiology, Poland; Lucjan Szponar, MD;PhD, National Food and Nutrition Institute, Poland; Heli Tapanainen, MSc, National Institute for Health and Welfare, Finland; Robert Templeton; Anastasia Thanopoulou, MD, PhD, Diabetes Center, 2nd Department of Internal Medicine, National University of Athens, Hippokration General Hospital, Greece; Holmfridur Thorgeirsdóttir, MSc, Directorate of Health, Iceland; Inga Thorsdottir; Antonia Trichopoulou, MD, Hellenic Health Foundation, Greece; Shoichiro Tsugane, MD, PhD, National Cancer Center, Japan; Aida Turrini, MD, National Research Institute on Food and Nutrition, Italy; Sirje Vaask, PhD, Tallinn University of Technology, Estonia; Coline van Oosterhout, National Institute for Public Health and the Environment, Netherlands; J Lennert Veerman, PhD, The University of Queensland, Australia; Nowak Verena; Anna Waskiewicz, MSc, PhD., Institute of Cardiology, Department of Cardiovascular Diseases Epidemiology, Prevention and Health Promotion, Poland; Sahar Zaghloul, PhD., National Nutrition Institute, Egypt; Gábor Zajkás, MD, National Institute of Food and Nutrition Sciences, Hungary.

This work was undertaken as part of the Global Burden of Diseases, Injuries, and Risk Factors Study. The results in this paper are prepared independently of the final estimates of the Global Burden of Diseases, Injuries, and Risk Factors study. We thank the Russia Longitudinal Monitoring Survey Phase 2, funded by the USAID and NIH (R01-HD38700), Higher School of Economics and Pension Fund of Russia, and the University of North Carolina Population Center (5 R24 HD050924; Source: "Russia Longitudinal Monitoring survey, RLMS-HSE», conducted by HSE and ZAO "Demoscope" together with Carolina Population Center, University of North Carolina at Chapel Hill and the Institute of Sociology RAS (RLMSHSE sites: http://www.cpc.unc.edu/projects/rlms, http://www.hse. ru/org/hse/rlms) for sharing the data with us. We thank Barbara Bowman, MS, PhD, National Center for Chronic Disease Prevention and Health Promotion, Centers for Disease Control and Prevention, Atlanta, GA, USA; Patricia Constante Jamie, PhD, School of Public Health, University of São Paulo, Sao Paolo, Brazil; Karen Lock, $\mathrm{PhD}$, London School of Hygiene and Tropical Medicine, London, $\mathrm{UK}$; and Joceline Pomerleau, PhD, London School of Hygiene and Tropical Medicine, London, UK for advising and guidance on initial search strategy. We thank Louise Dekker, MSc, Jenna Golan, MPH, Shadi Kalantarian, MD, MPH, Liesbeth Smit, PhD, and Georgina Waweru Harvard School of Public Health, Boston, MA, USA for data collection.

\section{Sources of Funding}

Dr Singh was supported by a T32 Training Grant in Academic Nutrition (DK007703) from the National Institute of Diabetes and Digestive and Kidney Diseases, and a K99/R00 Pathway to Independence Award (1K99HL124321) from the National Heart, Lung, and Blood Institute, National Institutes of Health. Initial data collection for this work was supported by a grant from the Bill \& Melinda Gates Foundation to the 2010 Global Burden of Diseases study. The funding sources had no role in the design or conduct of the study; collection, management, analysis, or interpretation of the data; or preparation, review, approval, or submission of the manuscript. All authors had access to all data sources and have responsibility for the contents of the report and the decision to submit for publication. The authors alone are responsible for the views expressed in this publication.

\section{Disclosures}

Dr Mozaffarian reports ad hoc travel reimbursement or honoraria for 1-time scientific presentations or reviews on diet and cardiometabolic diseases from Bunge and Haas Avocado Board; ad hoc consulting fees from Nutrition Impact, Amarin, Astra Zeneca, Boston Heart Diagnostics, GOED, and Life Sciences Research Organization; and membership, Unilever North America Scientific Advisory Board. The other authors report no conflicts. 


\section{References}

1. Lozano R, Naghavi M, Foreman K, Lim S, Shibuya K, Aboyans V, Abraham J, Adair T, Aggarwal R, Ahn SY, Alvarado M, Anderson HR, Anderson LM, Andrews KG, Atkinson C, Baddour LM, Barker-Collo S, Bartels DH, Bell ML, Benjamin EJ, Bennett D, Bhalla K, Bikbov B, Bin Abdulhak A, Birbeck G, Blyth F, Bolliger I, Boufous S, Bucello C, Burch M, Burney P, Carapetis J, Chen H, Chou D, Chugh SS, Coffeng LE, Colan SD, Colquhoun S, Colson KE, Condon J, Connor MD, Cooper LT, Corriere M, Cortinovis M, de Vaccaro KC, Couser W, Cowie BC, Criqui MH, Cross M, Dabhadkar KC, Dahodwala N, De Leo D, Degenhardt L, Delossantos A, Denenberg J, Des Jarlais DC, Dharmaratne SD, Dorsey ER, Driscoll T, Duber H, Ebel B, Erwin PJ, Espindola P, Ezzati M, Feigin V, Flaxman AD, Forouzanfar MH, Fowkes FG, Franklin R, Fransen M, Freeman MK, Gabriel SE, Gakidou E, Gaspari F, Gillum RF, Gonzalez-Medina D, Halasa YA, Haring D, Harrison JE, Havmoeller R, Hay RJ, Hoen B, Hotez PJ, Hoy D, Jacobsen KH, James SL, Jasrasaria R, Jayaraman S, Johns N, Karthikeyan G, Kassebaum N, Keren A, Khoo JP, Knowlton LM, Kobusingye O, Koranteng A, Krishnamurthi R, Lipnick M, Lipshultz SE, Ohno SL, Mabweijano J, MacIntyre MF, Mallinger L, March L, Marks GB, Marks R, Matsumori A, Matzopoulos R, Mayosi BM, McAnulty JH, McDermott MM, McGrath J, Mensah GA, Merriman TR, Michaud C, Miller M, Miller TR, Mock C, Mocumbi AO, Mokdad AA, Moran A, Mulholland K, Nair MN, Naldi L, Narayan KM, Nasseri K, Norman P, O'Donnell M, Omer SB, Ortblad K, Osborne R, Ozgediz D, Pahari B, Pandian JD, Rivero AP, Padilla RP, Perez-Ruiz F, Perico N, Phillips D, Pierce K, Pope CA 3rd, Porrini E, Pourmalek F, Raju M, Ranganathan D, Rehm JT, Rein DB, Remuzzi G, Rivara FP, Roberts T, De León FR, Rosenfeld LC, Rushton L, Sacco RL, Salomon JA, Sampson U, Sanman E, Schwebel DC, Segui-Gomez M, Shepard DS, Singh D, Singleton J, Sliwa K, Smith E, Steer A, Taylor JA, Thomas B, Tleyjeh IM, Towbin JA, Truelsen T, Undurraga EA, Venketasubramanian N, Vijayakumar L, Vos T, Wagner GR, Wang M, Wang W, Watt K, Weinstock MA, Weintraub R, Wilkinson JD, Woolf AD, Wulf S, Yeh PH, Yip P, Zabetian A, Zheng ZJ, Lopez AD, Murray CJ, AlMazroa MA, Memish ZA. Global and regional mortality from 235 causes of death for 20 age groups in 1990 and 2010: a systematic analysis for the Global Burden of Disease Study 2010. Lancet. 2012;380:2095-2128. doi: 10.1016/ S0140-6736(12)61728-0.

2. de Ruyter JC, Olthof MR, Seidell JC, Katan MB. A trial of sugar-free or sugar-sweetened beverages and body weight in children. $N$ Engl J Med. 2012;367:1397-1406. doi: 10.1056/NEJMoa1203034.

3. Ebbeling CB, Feldman HA, Chomitz VR, Antonelli TA, Gortmaker SL, Osganian SK, Ludwig DS. A randomized trial of sugar-sweetened beverages and adolescent body weight. N Engl J Med. 2012;367:1407-1416. doi: 10.1056/NEJMoa1203388.

4. Qi Q, Chu AY, Kang JH, Jensen MK, Curhan GC, Pasquale LR, Ridker PM, Hunter DJ, Willett WC, Rimm EB, Chasman DI, Hu FB, Qi L. Sugar-sweetened beverages and genetic risk of obesity. $N$ Engl J Med. 2012;367:1387-1396. doi: 10.1056/NEJMoa1203039.

5. Malik VS, Popkin BM, Bray GA, Després JP, Hu FB. Sugarsweetened beverages, obesity, type 2 diabetes mellitus, and cardiovascular disease risk. Circulation. 2010;121:1356-1364. doi: 10.1161/ CIRCULATIONAHA.109.876185.

6. Basu S, McKee M, Galea G, Stuckler D. Relationship of soft drink consumption to global overweight, obesity, and diabetes: a cross-national analysis of 75 countries. Am J Public Health. 2013;103:2071-2077. doi: 10.2105/AJPH.2012.300974

7. Stevens GA, Singh GM, Lu Y, Danaei G, Lin JK, Finucane MM, Bahalim AN, McIntire RK, Gutierrez HR, Cowan M, Paciorek CJ, Farzadfar F, Riley L, Ezzati M; Global Burden of Metabolic Risk Factors of Chronic Diseases Collaborating Group (Body Mass Index). National, regional, and global trends in adult overweight and obesity prevalences. Popul Health Metr. 2012;10:22. doi: 10.1186/1478-7954-10-22.

8. Finucane MM, Stevens GA, Cowan MJ, Danaei G, Lin JK, Paciorek CJ, Singh GM, Gutierrez HR, Lu Y, Bahalim AN, Farzadfar F, Riley LM, Ezzati M; Global Burden of Metabolic Risk Factors of Chronic Diseases Collaborating Group (Body Mass Index). National, regional, and global trends in body-mass index since 1980: systematic analysis of health examination surveys and epidemiological studies with 960 country-years and 9.1 million participants. Lancet. 2011;377:557-567. doi: 10.1016/ S0140-6736(10)62037-5.

9. Barquera S, Hernandez-Barrera L, Tolentino ML, Espinosa J, Ng SW, Rivera JA, Popkin BM. Energy intake from beverages is increasing among
Mexican adolescents and adults. J Nutr. 2008;138:2454-2461. doi: 10.3945/jn.108.092163.

10. Bremer AA, Byrd RS, Auinger P. Racial trends in sugar-sweetened beverage consumption among US adolescents: 1988-2004. Int J Adolesc Med Health. 2011;23:279-286.

11. Denova-Gutiérrez E, Talavera JO, Huitrón-Bravo G, Méndez-Hernández $\mathrm{P}$, Salmerón J. Sweetened beverage consumption and increased risk of metabolic syndrome in Mexican adults. Public Health Nutr. 2010;13:835842. doi: $10.1017 / \mathrm{S} 1368980009991145$.

12. Fagherazzi G, Vilier A, Saes Sartorelli D, Lajous M, Balkau B, ClavelChapelon F. Consumption of artificially and sugar-sweetened beverages and incident type 2 diabetes in the Etude Epidemiologique aupres des femmes de la Mutuelle Generale de l'Education Nationale-European Prospective Investigation into Cancer and Nutrition cohort. Am J Clin Nutr. 2013;97:517-523. doi: 10.3945/ajcn.112.050997.

13. Han E, Kim TH, Powell LM. Beverage consumption and individuallevel associations in South Korea. BMC Public Health. 2013;13:195. doi: 10.1186/1471-2458-13-195.

14. Jimenez-Aguilar A, Flores M, Shamah-Levy T. Sugar-sweetened beverages consumption and BMI in Mexican adolescents: Mexican National Health and Nutrition Survey 2006. Salud Publica Mex. 2009;51(suppl 4):S604-S612.

15. Khosravi-Boroujeni H, Sarrafzadegan N, Mohammadifard N, Alikhasi H, Sajjadi F, Asgari S, Esmaillzadeh A. Consumption of sugar-sweetened beverages in relation to the metabolic syndrome among Iranian adults. Obes Facts. 2012;5:527-537. doi: 10.1159/000341886.

16. Kim YH, Abris GP, Sung MK, Lee JE. Consumption of sugar-sweetened beverages and blood pressure in the United States: the national health and nutrition examination survey 2003-2006. Clin Nutr Res. 2012;1:85-93. doi: 10.7762/cnr.2012.1.1.85.

17. Nikpartow N, Danyliw AD, Whiting SJ, Lim H, Vatanparast H. Fruit drink consumption is associated with overweight and obesity in Canadian women. Can J Public Health. 2012;103:178-182.

18. Shang XW, Liu AL, Zhang Q, Hu XQ, Du SM, Ma J, Xu GF, Li Y, Guo HW, Du L, Li TY, Ma GS. Report on childhood obesity in China (9): sugar-sweetened beverages consumption and obesity. Biomed Environ Sci. 2012;25:125-132. doi: 10.3967/0895-3988.2012.02.001.

19. Lim SS, Vos T, Flaxman AD, Danaei G, Shibuya K, Adair-Rohani $\mathrm{H}$, Amann M, Anderson HR, Andrews KG, Aryee M, Atkinson C, Bacchus LJ, Bahalim AN, Balakrishnan K, Balmes J, Barker-Collo S, Baxter A, Bell ML, Blore JD, Blyth F, Bonner C, Borges G, Bourne R, Boussinesq M, Brauer M, Brooks P, Bruce NG, Brunekreef B, BryanHancock C, Bucello C, Buchbinder R, Bull F, Burnett RT, Byers TE, Calabria B, Carapetis J, Carnahan E, Chafe Z, Charlson F, Chen H, Chen JS, Cheng AT, Child JC, Cohen A, Colson KE, Cowie BC, Darby S, Darling S, Davis A, Degenhardt L, Dentener F, Des Jarlais DC, Devries K, Dherani M, Ding EL, Dorsey ER, Driscoll T, Edmond K, Ali SE, Engell RE, Erwin PJ, Fahimi S, Falder G, Farzadfar F, Ferrari A, Finucane MM, Flaxman S, Fowkes FG, Freedman G, Freeman MK, Gakidou E, Ghosh S, Giovannucci E, Gmel G, Graham K, Grainger R, Grant B, Gunnell D, Gutierrez HR, Hall W, Hoek HW, Hogan A, Hosgood HD 3rd, Hoy D, Hu H, Hubbell BJ, Hutchings SJ, Ibeanusi SE, Jacklyn GL, Jasrasaria R, Jonas JB, Kan H, Kanis JA, Kassebaum N, Kawakami N, Khang YH, Khatibzadeh S, Khoo JP, Kok C, Laden F, Lalloo R, Lan Q, Lathlean T, Leasher JL, Leigh J, Li Y, Lin JK, Lipshultz SE, London S, Lozano R, Lu Y, Mak J, Malekzadeh R, Mallinger L, Marcenes W, March L, Marks R, Martin R, McGale P, McGrath J, Mehta S, Mensah GA, Merriman TR, Micha R, Michaud C, Mishra V, Mohd Hanafiah K, Mokdad AA, Morawska L, Mozaffarian D, Murphy T, Naghavi M, Neal B, Nelson PK, Nolla JM, Norman R, Olives C, Omer SB, Orchard J, Osborne R, Ostro B, Page A, Pandey KD, Parry CD, Passmore E, Patra J, Pearce N, Pelizzari PM, Petzold M, Phillips MR, Pope D, Pope CA 3rd, Powles J, Rao M, Razavi H, Rehfuess EA, Rehm JT, Ritz B, Rivara FP, Roberts T, Robinson C, RodriguezPortales JA, Romieu I, Room R, Rosenfeld LC, Roy A, Rushton L, Salomon JA, Sampson U, Sanchez-Riera L, Sanman E, Sapkota A, Seedat S, Shi P, Shield K, Shivakoti R, Singh GM, Sleet DA, Smith E, Smith KR, Stapelberg NJ, Steenland K, Stöckl H, Stovner LJ, Straif K, Straney L, Thurston GD, Tran JH, Van Dingenen R, van Donkelaar A, Veerman JL, Vijayakumar L, Weintraub R, Weissman MM, White RA, Whiteford H, Wiersma ST, Wilkinson JD, Williams HC, Williams W, Wilson N, Woolf AD, Yip P, Zielinski JM, Lopez AD, Murray CJ, Ezzati M, AlMazroa MA, Memish ZA. A comparative risk assessment of burden of disease and injury attributable to 67 risk factors and risk factor clusters in 21 regions, 1990-2010: a systematic analysis for the 
Global Burden of Disease Study 2010. Lancet. 2012;380:2224-2260. doi: 10.1016/S0140-6736(12)61766-8.

20. Murray CJ, Ezzati M, Flaxman AD, Lim S, Lozano R, Michaud C, Naghavi M, Salomon JA, Shibuya K, Vos T, Lopez AD. GBD 2010: a multi-investigator collaboration for global comparative descriptive epidemiology. Lancet. 2012;380:2055-2058. doi: 10.1016/ S0140-6736(12)62134-5.

21. Murray CJ, Ezzati M, Flaxman AD, Lim S, Lozano R, Michaud C, Naghavi M, Salomon JA, Shibuya K, Vos T, Wikler D, Lopez AD. GBD 2010: design, definitions, and metrics. Lancet. 2012;380:2063-2066. doi: 10.1016/S0140-6736(12)61899-6.

22. Greenwood DC, Threapleton DE, Evans CE, Cleghorn CL, Nykjaer C, Woodhead C, Burley VJ. Association between sugar-sweetened and artificially sweetened soft drinks and type 2 diabetes: systematic review and dose-response meta-analysis of prospective studies. $\mathrm{Br} \mathrm{J} \mathrm{Nutr}$. 2014;112:725-734. doi: 10.1017/S0007114514001329.

23. Mozaffarian D, Hao T, Rimm EB, Willett WC, Hu FB. Changes in diet and lifestyle and long-term weight gain in women and men. N Engl J Med. 2011;364:2392-2404.

24. Singh GM, Danaei G, Farzadfar F, Stevens GA, Woodward M, Wormser D, Kaptoge S, Whitlock G, Qiao Q, Lewington S, Di Angelantonio E, Vander Hoorn S, Lawes CM, Ali MK, Mozaffarian D, Ezzati M; Global Burden of Metabolic Risk Factors of Chronic Diseases Collaborating Group; Asia-Pacific Cohort Studies Collaboration (APCSC); Diabetes Epidemiology: Collaborative analysis of Diagnostic criteria in Europe (DECODE); Emerging Risk Factor Collaboration (ERFC); Prospective Studies Collaboration (PSC). The age-specific quantitative effects of metabolic risk factors on cardiovascular diseases and diabetes: a pooled analysis. PLoS One. 2013;8:e65174. doi: 10.1371/journal.pone.0065174.

25. Renehan AG, Tyson M, Egger M, Heller RF, Zwahlen M. Body-mass index and incidence of cancer: a systematic review and meta-analysis of prospective observational studies. Lancet. 2008;371:569-578. doi: 10.1016/S0140-6736(08)60269-X

26. Singh GM, Micha R, Khatibzadeh S, Shi P, Lim S, Andrews KG, Engell RE, Ezzati M, Mozaffarian D. Global, regional, and national consumption levels of sugar-sweetened beverages, fruit juice, and milk: a systematic assessment of beverage intake in 187 countries. PLoS One. In press.

27. FAO. U.N. Food and Agriculture Organization Food Balance Sheets. http://faostatfaoorg/site/354/defaultaspx. 2013.

28. Willett W. Nutritional Epidemiology. Chapter 11: "Implications for Total Energy Intake for Epidemiologic Analyses.” New York: Oxford University Press; 1998.

29. Khatibzadeh S, Micha R, Afshin A, Rao M, Yakoob MY, Mozaffarian D. Major dietary risk factors for chronic diseases: a systematic review of the current evidence for causal effects and effect sizes. Circulation. 2012;125:AP060.

30. Malik VS, Pan A, Willett WC, Hu FB. Sugar-sweetened beverages and weight gain in children and adults: a systematic review and meta-analysis. Am J Clin Nutr. 2013;98:1084-1102. doi: 10.3945/ajcn.113.058362.

31. Malik VS, Popkin BM, Bray GA, Després JP, Willett WC, Hu FB. Sugarsweetened beverages and risk of metabolic syndrome and type 2 diabetes: a meta-analysis. Diabetes Care. 2010;33:2477-2483. doi: 10.2337/ dc10-1079.

32. EPIC-InterAct. Consumption of sweet beverages and type 2 diabetes incidence in European adults: results from EPIC-InterAct. Diabetologia. 2013;56:1520-1530

33. Fung TT, Malik V, Rexrode KM, Manson JE, Willett WC, Hu FB Sweetened beverage consumption and risk of coronary heart disease in women. Am J Clin Nutr. 2009;89:1037-1042. doi: 10.3945/ ajcn.2008.27140.

34. Malik AH, Akram Y, Shetty S, Malik SS, Yanchou Njike V. Impact of sugarsweetened beverages on blood pressure. Am J Cardiol. 2014;113:1574 1580. doi: 10.1016/j.amjcard.2014.01.437.

35. Schulze MB, Manson JE, Ludwig DS, Colditz GA, Stampfer MJ, Willett WC, Hu FB. Sugar-sweetened beverages, weight gain, and incidence of type 2 diabetes in young and middle-aged women. JAMA. 2004;292:927934. doi: 10.1001/jama.292.8.927.

36. Danaei G, Ding EL, Mozaffarian D, Taylor B, Rehm J, Murray CJ, Ezzati M. The preventable causes of death in the United States: comparative risk assessment of dietary, lifestyle, and metabolic risk factors. PLoS Med. 2009;6:e1000058. doi: 10.1371/journal.pmed.1000058.

37. Murray CJ, Vos T, Lozano R, Naghavi M, Flaxman AD, Michaud C, Ezzati M, Shibuya K, Salomon JA, Abdalla S, Aboyans V, Abraham J, Ackerman I, Aggarwal R, Ahn SY, Ali MK, Alvarado M, Anderson HR, Anderson LM, Andrews KG, Atkinson C, Baddour LM, Bahalim
AN, Barker-Collo S, Barrero LH, Bartels DH, Basáñez MG, Baxter A, Bell ML, Benjamin EJ, Bennett D, Bernabé E, Bhalla K, Bhandari B, Bikbov B, Bin Abdulhak A, Birbeck G, Black JA, Blencowe H, Blore JD, Blyth F, Bolliger I, Bonaventure A, Boufous S, Bourne R, Boussinesq M, Braithwaite T, Brayne C, Bridgett L, Brooker S, Brooks P, Brugha TS, Bryan-Hancock C, Bucello C, Buchbinder R, Buckle G, Budke CM, Burch M, Burney P, Burstein R, Calabria B, Campbell B, Canter CE, Carabin H, Carapetis J, Carmona L, Cella C, Charlson F, Chen H, Cheng AT, Chou D, Chugh SS, Coffeng LE, Colan SD, Colquhoun S, Colson KE, Condon J, Connor MD, Cooper LT, Corriere M, Cortinovis M, de Vaccaro KC, Couser W, Cowie BC, Criqui MH, Cross M, Dabhadkar KC, Dahiya M, Dahodwala N, Damsere-Derry J, Danaei G, Davis A, De Leo D, Degenhardt L, Dellavalle R, Delossantos A, Denenberg J, Derrett S, Des Jarlais DC, Dharmaratne SD, Dherani M, Diaz-Torne C, Dolk H, Dorsey ER, Driscoll T, Duber H, Ebel B, Edmond K, Elbaz A, Ali SE, Erskine H, Erwin PJ, Espindola P, Ewoigbokhan SE, Farzadfar F, Feigin V, Felson DT, Ferrari A, Ferri CP, Fèvre EM, Finucane MM, Flaxman S, Flood L, Foreman K, Forouzanfar MH, Fowkes FG, Fransen M, Freeman MK, Gabbe BJ, Gabriel SE, Gakidou E, Ganatra HA, Garcia B, Gaspari F, Gillum RF, Gmel G, Gonzalez-Medina D, Gosselin R, Grainger R, Grant B, Groeger J, Guillemin F, Gunnell D, Gupta R, Haagsma J, Hagan H, Halasa YA, Hall W, Haring D, Haro JM, Harrison JE, Havmoeller R, Hay RJ, Higashi H, Hill C, Hoen B, Hoffman H, Hotez PJ, Hoy D, Huang JJ, Ibeanusi SE, Jacobsen KH, James SL, Jarvis D, Jasrasaria R, Jayaraman S, Johns N, Jonas JB, Karthikeyan G, Kassebaum N, Kawakami N, Keren A, Khoo JP, King CH, Knowlton LM, Kobusingye O, Koranteng A, Krishnamurthi R, Laden F, Lalloo R, Laslett LL, Lathlean T, Leasher JL, Lee YY, Leigh J, Levinson D, Lim SS, Limb E, Lin JK, Lipnick M, Lipshultz SE, Liu W, Loane M, Ohno SL, Lyons R, Mabweijano J, MacIntyre MF, Malekzadeh R, Mallinger L, Manivannan S, Marcenes W, March L, Margolis DJ, Marks GB, Marks R, Matsumori A, Matzopoulos R, Mayosi BM, McAnulty JH, McDermott MM, McGill N, McGrath J, Medina-Mora ME, Meltzer M, Mensah GA, Merriman TR, Meyer AC, Miglioli V, Miller M, Miller TR, Mitchell PB, Mock C, Mocumbi AO, Moffitt TE, Mokdad AA, Monasta L, Montico M, Moradi-Lakeh M, Moran A, Morawska L, Mori R, Murdoch ME, Mwaniki MK, Naidoo K, Nair MN, Naldi L, Narayan KM, Nelson PK, Nelson RG, Nevitt MC, Newton CR, Nolte S, Norman P, Norman R, O'Donnell M, O'Hanlon S, Olives C, Omer SB, Ortblad K, Osborne R, Ozgediz D, Page A, Pahari B, Pandian JD, Rivero AP, Patten SB, Pearce N, Padilla RP, Perez-Ruiz F, Perico N, Pesudovs K, Phillips D, Phillips MR, Pierce K, Pion S, Polanczyk GV, Polinder S, Pope CA 3rd, Popova S, Porrini E, Pourmalek F, Prince M, Pullan RL, Ramaiah KD, Ranganathan D, Razavi H, Regan M, Rehm JT, Rein DB, Remuzzi G, Richardson K, Rivara FP, Roberts T, Robinson C, De Leòn FR, Ronfani L, Room R, Rosenfeld LC, Rushton L, Sacco RL, Saha S, Sampson U, Sanchez-Riera L, Sanman E, Schwebel DC, Scott JG, Segui-Gomez M, Shahraz S, Shepard DS, Shin H, Shivakoti R, Singh D, Singh GM, Singh JA, Singleton J, Sleet DA, Sliwa K, Smith E, Smith JL, Stapelberg NJ, Steer A, Steiner T, Stolk WA, Stovner LJ, Sudfeld C, Syed S, Tamburlini G, Tavakkoli M, Taylor HR, Taylor JA, Taylor WJ, Thomas B, Thomson WM, Thurston GD, Tleyjeh IM, Tonelli M, Towbin JA, Truelsen T, Tsilimbaris MK, Ubeda C, Undurraga EA, van der Werf MJ, van Os J, Vavilala MS, Venketasubramanian N, Wang M, Wang W, Watt K, Weatherall DJ, Weinstock MA, Weintraub R, Weisskopf MG, Weissman MM, White RA, Whiteford H, Wiebe N, Wiersma ST, Wilkinson JD, Williams HC, Williams SR, Witt E, Wolfe F, Woolf AD, Wulf S, Yeh PH, Zaidi AK, Zheng ZJ, Zonies D, Lopez AD, AlMazroa MA, Memish ZA. Disability-adjusted life years (DALYs) for 291 diseases and injuries in 21 regions, 1990-2010: a systematic analysis for the Global Burden of Disease Study 2010. Lancet. 2012;380:2197-2223. doi: 10.1016/ S0140-6736(12)61689-4

38. Vos T, Flaxman AD, Naghavi M, Lozano R, Michaud C, Ezzati M, Shibuya K, Salomon JA, Abdalla S, Aboyans V, Abraham J, Ackerman I, Aggarwal R, Ahn SY, Ali MK, Alvarado M, Anderson HR, Anderson LM, Andrews KG, Atkinson C, Baddour LM, Bahalim AN, BarkerCollo S, Barrero LH, Bartels DH, Basáñez MG, Baxter A, Bell ML, Benjamin EJ, Bennett D, Bernabé E, Bhalla K, Bhandari B, Bikbov B, Bin Abdulhak A, Birbeck G, Black JA, Blencowe H, Blore JD, Blyth F, Bolliger I, Bonaventure A, Boufous S, Bourne R, Boussinesq M, Braithwaite T, Brayne C, Bridgett L, Brooker S, Brooks P, Brugha TS, Bryan-Hancock C, Bucello C, Buchbinder R, Buckle G, Budke CM, Burch M, Burney P, Burstein R, Calabria B, Campbell B, Canter CE, 
Carabin H, Carapetis J, Carmona L, Cella C, Charlson F, Chen H, Cheng AT, Chou D, Chugh SS, Coffeng LE, Colan SD, Colquhoun S, Colson KE, Condon J, Connor MD, Cooper LT, Corriere M, Cortinovis M, de Vaccaro KC, Couser W, Cowie BC, Criqui MH, Cross M, Dabhadkar KC, Dahiya M, Dahodwala N, Damsere-Derry J, Danaei G, Davis A, De Leo D, Degenhardt L, Dellavalle R, Delossantos A, Denenberg J, Derrett S, Des Jarlais DC, Dharmaratne SD, Dherani M, DiazTorne C, Dolk H, Dorsey ER, Driscoll T, Duber H, Ebel B, Edmond K, Elbaz A, Ali SE, Erskine H, Erwin PJ, Espindola P, Ewoigbokhan SE, Farzadfar F, Feigin V, Felson DT, Ferrari A, Ferri CP, Fèvre EM, Finucane MM, Flaxman S, Flood L, Foreman K, Forouzanfar MH, Fowkes FG, Franklin R, Fransen M, Freeman MK, Gabbe BJ, Gabriel SE, Gakidou E, Ganatra HA, Garcia B, Gaspari F, Gillum RF, Gmel G, Gosselin R, Grainger R, Groeger J, Guillemin F, Gunnell D, Gupta R, Haagsma J, Hagan H, Halasa YA, Hall W, Haring D, Haro JM, Harrison JE, Havmoeller R, Hay RJ, Higashi H, Hill C, Hoen B, Hoffman H, Hotez PJ, Hoy D, Huang JJ, Ibeanusi SE, Jacobsen KH, James SL, Jarvis D, Jasrasaria R, Jayaraman S, Johns N, Jonas JB, Karthikeyan G, Kassebaum N, Kawakami N, Keren A, Khoo JP, King CH, Knowlton LM, Kobusingye O, Koranteng A, Krishnamurthi R, Lalloo R, Laslett LL, Lathlean T, Leasher JL, Lee YY, Leigh J, Lim SS, Limb E, Lin JK, Lipnick M, Lipshultz SE, Liu W, Loane M, Ohno SL, Lyons R, Ma J, Mabweijano J, MacIntyre MF, Malekzadeh R, Mallinger L, Manivannan S, Marcenes W, March L, Margolis DJ, Marks GB, Marks R, Matsumori A, Matzopoulos R, Mayosi BM, McAnulty JH, McDermott MM, McGill N, McGrath J, Medina-Mora ME, Meltzer M, Mensah GA, Merriman TR, Meyer AC, Miglioli V, Miller M, Miller TR, Mitchell PB, Mocumbi AO, Moffitt TE, Mokdad AA, Monasta L, Montico M, Moradi-Lakeh M, Moran A, Morawska L, Mori R, Murdoch ME, Mwaniki MK, Naidoo K, Nair MN, Naldi L, Narayan KM, Nelson PK, Nelson RG, Nevitt MC, Newton CR, Nolte S, Norman P, Norman R, O'Donnell M, O'Hanlon S, Olives C, Omer SB, Ortblad K, Osborne R, Ozgediz D, Page A, Pahari B, Pandian JD, Rivero AP, Patten SB, Pearce N, Padilla RP, Perez-Ruiz F, Perico N, Pesudovs K, Phillips D, Phillips MR, Pierce K, Pion S, Polanczyk GV, Polinder S, Pope CA 3rd, Popova S, Porrini E, Pourmalek F, Prince M, Pullan RL, Ramaiah KD, Ranganathan D, Razavi H, Regan M, Rehm JT, Rein DB, Remuzzi G, Richardson K, Rivara FP, Roberts T, Robinson C, De Leòn FR, Ronfani L, Room R, Rosenfeld LC, Rushton L, Sacco RL, Saha S, Sampson U, Sanchez-Riera L, Sanman E, Schwebel DC, Scott JG, Segui-Gomez M, Shahraz S, Shepard DS, Shin H, Shivakoti R, Singh D, Singh GM, Singh JA, Singleton J, Sleet DA, Sliwa K, Smith E, Smith JL, Stapelberg NJ, Steer A, Steiner T, Stolk WA, Stovner LJ, Sudfeld C, Syed S, Tamburlini G, Tavakkoli M, Taylor HR, Taylor JA, Taylor WJ, Thomas B, Thomson WM, Thurston GD, Tleyjeh IM, Tonelli M, Towbin JA, Truelsen T, Tsilimbaris MK, Ubeda C, Undurraga EA, van der Werf MJ, van Os J, Vavilala MS, Venketasubramanian N, Wang M, Wang W, Watt K, Weatherall DJ, Weinstock MA, Weintraub R, Weisskopf MG, Weissman MM, White RA, Whiteford H, Wiersma ST, Wilkinson JD, Williams HC, Williams SR, Witt E, Wolfe F, Woolf AD, Wulf S, Yeh $\mathrm{PH}$, Zaidi AK, Zheng ZJ, Zonies D, Lopez AD, Murray CJ, AlMazroa MA, Memish ZA. Years lived with disability (YLDs) for 1160 sequelae of 289 diseases and injuries 1990-2010: a systematic analysis for the Global Burden of Disease Study 2010. Lancet. 2012;380:2163-2196. doi: 10.1016/S0140-6736(12)61729-2.

39. Salomon JA, Vos T, Hogan DR, Gagnon M, Naghavi M, Mokdad A, Begum N, Shah R, Karyana M, Kosen S, Farje MR, Moncada G, Dutta A, Sazawal S, Dyer A, Seiler J, Aboyans V, Baker L, Baxter A, Benjamin EJ, Bhalla K, Bin Abdulhak A, Blyth F, Bourne R, Braithwaite T, Brooks P, Brugha TS, Bryan-Hancock C, Buchbinder R, Burney P, Calabria B, Chen H, Chugh SS, Cooley R, Criqui MH, Cross M, Dabhadkar KC, Dahodwala N, Davis A, Degenhardt L, DíazTorné C, Dorsey ER, Driscoll T, Edmond K, Elbaz A, Ezzati M, Feigin V, Ferri CP, Flaxman AD, Flood L, Fransen M, Fuse K, Gabbe BJ, Gillum RF, Haagsma J, Harrison JE, Havmoeller R, Hay RJ, Hel-Baqui A, Hoek HW, Hoffman H, Hogeland E, Hoy D, Jarvis D, Karthikeyan
G, Knowlton LM, Lathlean T, Leasher JL, Lim SS, Lipshultz SE, Lopez AD, Lozano R, Lyons R, Malekzadeh R, Marcenes W, March L, Margolis DJ, McGill N, McGrath J, Mensah GA, Meyer AC, Michaud C, Moran A, Mori R, Murdoch ME, Naldi L, Newton CR, Norman R, Omer SB, Osborne R, Pearce N, Perez-Ruiz F, Perico N, Pesudovs K, Phillips D, Pourmalek F, Prince M, Rehm JT, Remuzzi G, Richardson K, Room R, Saha S, Sampson U, Sanchez-Riera L, Segui-Gomez M, Shahraz S, Shibuya K, Singh D, Sliwa K, Smith E, Soerjomataram I, Steiner T, Stolk WA, Stovner LJ, Sudfeld C, Taylor HR, Tleyjeh IM, van der Werf MJ, Watson WL, Weatherall DJ, Weintraub R, Weisskopf MG, Whiteford H, Wilkinson JD, Woolf AD, Zheng ZJ, Murray CJ, Jonas JB. Common values in assessing health outcomes from disease and injury: disability weights measurement study for the Global Burden of Disease Study 2010. Lancet. 2012;380:2129-2143. doi: 10.1016/ S0140-6736(12)61680-8.

40. Danaei G, Lu Y, Singh GM, Carnahan E, Stevens GA, Cowan MJ, Farzadfar F, Lin JK, Finucane MM, Rao M, Khang YH, Riley LM, Mozaffarian D, Lim SS, M E. Cardiovascular disease, chronic kidney disease, and diabetes mortality burden of cardiometabolic risk factors from 1980 to 2010: a comparative risk assessment. Lancet Diabetes Endocrinol. 2014;14:http://dx.doi.org/10.1016/ S2213-8587(14)70102-0.

41. Mozaffarian D, Fahimi S, Singh GM, Micha R, Khatibzadeh S, Engell RE, Lim S, Danaei G, Ezzati M, Powles J; Global Burden of Diseases Nutrition and Chronic Diseases Expert Group. Global sodium consumption and death from cardiovascular causes. N Engl J Med. 2014;371:624634. doi: 10.1056/NEJMoa1304127.

42. Danaei G, Finucane MM, Lu Y, Singh GM, Cowan MJ, Paciorek CJ, Lin JK, Farzadfar F, Khang YH, Stevens GA, Rao M, Ali MK, Riley LM, Robinson CA, Ezzati M; Global Burden of Metabolic Risk Factors of Chronic Diseases Collaborating Group (Blood Glucose). National, regional, and global trends in fasting plasma glucose and diabetes prevalence since 1980: systematic analysis of health examination surveys and epidemiological studies with 370 country-years and 2.7 million participants. Lancet. 2011;378:31-40. doi: 10.1016/ S0140-6736(11)60679-X.

43. Moise N, Cifuentes E, Orozco E, Willett W. Limiting the consumption of sugar sweetened beverages in Mexico's obesogenic environment: a qualitative policy review and stakeholder analysis. J Public Health Policy. 2011;32:458-475. doi: 10.1057/jphp.2011.39.

44. Barquera S, Campos I, Rivera JA. Mexico attempts to tackle obesity: the process, results, push backs and future challenges. Obes Rev. 2013;14 Suppl 2:69-78. doi: 10.1111/obr.12096.

45. Khatibzadeh S, Afshin A, Micha R, Shi P, Yawar Yakoob M, Singh GM, Rao M, Fahimi S, Ezzati M, Mozaffarian D. The impact of low consumption of fruits and vegetables on mortality from cardiovascular diseases in 187 countries. Circulation. 2014;129:AMP76.

46. IOM. Promoting Cardiovascular Health in the Developing World: A Critical Challenge to Achieve Global Health. Washington, DC: Institute of Medicine; 2010.

47. Brown CM, Dulloo AG, Yepuri G, Montani JP. Fructose ingestion acutely elevates blood pressure in healthy young humans. Am J Physiol Regul Integr Comp Physiol. 2008;294:R730-R737. doi: 10.1152/ajpregu.00680.2007.

48. Choi JW, Ford ES, Gao X, Choi HK. Sugar-sweetened soft drinks, diet soft drinks, and serum uric acid level: the Third National Health and Nutrition Examination Survey. Arthritis Rheum. 2008;59:109-116. doi: 10.1002/art.23245.

49. Dhingra R, Sullivan L, Jacques PF, Wang TJ, Fox CS, Meigs JB, D'Agostino RB, Gaziano JM, Vasan RS. Soft drink consumption and risk of developing cardiometabolic risk factors and the metabolic syndrome in middle-aged adults in the community. Circulation. 2007;116:480-488. doi: 10.1161/CIRCULATIONAHA.107.689935.

50. Liu S, Manson JE, Buring JE, Stampfer MJ, Willett WC, Ridker PM. Relation between a diet with a high glycemic load and plasma concentrations of high-sensitivity C-reactive protein in middle-aged women. Am J Clin Nutr. 2002;75:492-498. 


\section{CLINICAL PERSPECTIVE}

Sugar-sweetened beverages (SSBs) increase obesity and type 2 diabetes mellitus and are now consumed worldwide. Yet, the impact of SSBs on global diabetes mellitus- and obesity-related diseases by nation, age, and sex has not been reported. We developed a global model incorporating diverse data sources across 187 countries, by age and sex, on the following: SSB intake patterns from individual-level dietary surveys and national food availability; effects of SSBs on adiposity and diabetes mellitus from meta-analyses of longitudinal cohort studies; impact of adiposity on diabetes mellitus, cardiovascular disease, and cancers from meta-analyses of international pooling projects; and numbers of cause-specific national deaths from the Global Burden of Diseases study. Using a comparative risk assessment model, in 2010, we estimated 184000 deaths/y and 8.5 million disability-adjusted life-years per year attributable to SSBs worldwide; $75 \%$ of deaths and $85 \%$ of disability-adjusted life-years occurred in low- and middle-income countries. Tremendous geographic variation was evident, with the largest burdens in Latin America and the Caribbean, and the smallest burdens in East Asia. Proportional impacts (percentage of disease attributable to SSBs) were highest in young adults, particularly young men. In Mexico, 1 in 3 diabetes mellitus- and obesityrelated deaths in men $<44$ years of age were attributable to SSBs; and in the United States, 1 in 10 such deaths were attributable to SSBs. Given a dramatic inverse gradient between age and SSB intake in most nations, future health burdens could be even higher as younger populations age. Our findings highlight the large global health burdens, and the key geographic and demographic variation, as well, attributable to SSBs, providing the basis for targeted health policy and interventions. 